\title{
Are the CKM anomalies induced by vector-like quarks? Limits from flavor changing and Standard Model precision tests
}

\author{
B. Belfatto ${ }^{a, b}$ and Z. Berezhiani ${ }^{c, d}$ \\ ${ }^{a}$ Dipartimento di Fisica "E. Fermi", Università di Pisa, \\ Largo Bruno Pontecorvo 3, I-56127 Pisa, Italy \\ ${ }^{b}$ INFN, Sezione di Pisa, Largo Bruno Pontecorvo 3, I-56127 Pisa, Italy \\ ${ }^{c}$ Dipartimento di Fisica e Chimica, Università di L'Aquila, 67100 Coppito, L'Aquila, Italy \\ ${ }^{d}$ INFN, Laboratori Nazionali del Gran Sasso, 67010 Assergi, L'Aquila, Italy
}

E-mail: benedetta.belfatto@gssi.it, zurab.berezhiani@lngs.infn.it

ABSTRACT: Recent high precision determinations of $V_{u s}$ and $V_{u d}$ indicate towards anomalies in the first row of the CKM matrix. Namely, determination of $V_{u d}$ from beta decays and of $V_{u s}$ from kaon decays imply a violation of first row unitarity at about $3 \sigma$ level. Moreover, there is tension between determinations of $V_{u s}$ obtained from leptonic $K \mu 2$ and semileptonic $K \ell 3$ kaon decays. These discrepancies can be explained if there exist extra vector-like quarks at the $\mathrm{TeV}$ scale, which have large enough mixings with the lighter quarks. In particular, extra vector-like weak singlets quarks can be thought as a solution to the CKM unitarity problem and an extra vector-like weak doublet can in principle resolve all tensions. The implications of this kind of mixings are examined against the flavour changing phenomena and SM precision tests. We consider separately the effects of an extra down-type isosinglet, up-type isosinglet and an isodoublet containing extra quarks of both up and down type, and determine available parameter spaces for each case. We find that the experimental constraints on flavor changing phenomena become more stringent with larger masses, so that the extra species should have masses no more than few TeV. Moreover, only one type of extra multiplet cannot entirely explain all the discrepancies, and some their combination is required, e.g. two species of isodoublet, or one isodoublet and one (up or down type) isosinglet. We show that these scenarios are testable with future experiments. Namely, if extra vector-like quarks are responsible for CKM anomalies, then at least one of them should be found at scale of few $\mathrm{TeV}$, and anomalous weak isospin violating $Z$-boson couplings with light quarks should be detected if the experimental precision on $Z$ hadronic decay rate is improved by a factor of 2 or so.

Keywords: Beyond Standard Model, Kaon Physics

ArXIV EPRINT: 2103.05549 


\section{Contents}

1 Introduction 1

2 Present situation in the determination of $\left|V_{u s}\right|$ and $\left|V_{u d}\right| \quad 3$

3 The SM with extra vector-like fermions $\quad 8$

4 Extra down-type isosinglet $\quad 16$

4.1 Limits from rare kaon decays 21

$\begin{array}{lll}\text { 4.1.1 } & K^{+} \rightarrow \pi^{+} \nu \bar{\nu} & 21\end{array}$

4.1.2 $K_{\mathrm{L}} \rightarrow \pi^{0} \nu \bar{\nu} \quad 24$

$\begin{array}{lll}\text { 4.1.3 } & K_{\mathrm{L}} \rightarrow \pi^{0} e^{+} e^{-} & 25\end{array}$

$\begin{array}{lll}\text { 4.1.4 } & K_{\mathrm{L}} \rightarrow \mu^{+} \mu^{-} & 27\end{array}$

4.1.5 $\quad K_{\mathrm{S}} \rightarrow \mu^{+} \mu^{-} \quad 28$

4.2 Limits from neutral mesons systems 29

$\begin{array}{lll}\text { 4.2.1 } & K^{0}-\bar{K}^{0} \text { mixing } & 29\end{array}$

4.2.2 $B_{d, s}^{0}-\bar{B}_{d, s}^{0}$ mixing $\quad 33$

4.2.3 $D^{0}-\bar{D}^{0}$ mixing $\quad 34$

4.3 Limits from rare $B$ mesons decays $\quad 36$

$\begin{array}{lll}\text { 4.3.1 } & B_{d, s}^{0} \rightarrow \mu^{+} \mu^{-} & 36\end{array}$

$\begin{array}{lll}\text { 4.3.2 } & \text { Rare semileptonic B decays } & 37\end{array}$

4.4 Limits from $Z$-boson physics 39

4.5 Summary of experimental limits $\quad 42$

$5 \quad$ Extra up-type isosinglet $\quad 46$

5.1 Limits from rare $D$ mesons decays 48

$\begin{array}{lll}5.2 & \text { Limits from neutral mesons systems } & 49\end{array}$

$\begin{array}{llr}5.2 .1 & D^{0}-\bar{D}^{0} \text { mixing } & 49\end{array}$

$\begin{array}{lll}5.2 .2 & K^{0}-\bar{K}^{0} \text { mixing } & 49\end{array}$

$\begin{array}{lll}5.2 .3 B_{s, d^{-}}^{0} \bar{B}_{s, d}^{0} \text { mixing } & 51\end{array}$

5.3 Limits from $Z$-boson physics $\quad 52$

5.4 Summary of experimental limits 53

6 Extra weak isodoublet $\quad 56$

6.1 Limits from flavour changing neutral currents 61

$\begin{array}{lll}6.2 & \text { Limits from } Z \text {-boson physics } & 63\end{array}$

6.3 Limits from low energy electroweak observables 64

6.4 Summary of experimental limits 66

$\begin{array}{lll}6.5 & \text { Possible extensions } & 68\end{array}$

$\begin{array}{lll}7 & \text { Conclusion } & 73\end{array}$ 


\section{Introduction}

The Standard Model $(\mathrm{SM}) \mathrm{SU}(3) \times \mathrm{SU}(2) \times \mathrm{U}(1)$ contains three fermion families with lefthanded (LH) components of quarks $q_{L i}=\left(u_{L}, d_{L}\right)_{i}$ and leptons $\ell_{L i}=\left(\nu_{L}, e_{L}\right)_{i}, i=1,2,3$, forming doublets and the right-handed $(\mathrm{RH})$ components $u_{R i}, d_{R i}, e_{R i}$ being singlets of isotopic symmetry $\mathrm{SU}(2)$ of weak interactions. The charged current weak interactions in terms of the quark mass eigenstates, up quarks $u, c, t$ and down quarks $d, s, b$, are described by the coupling

$$
\frac{g}{2 \sqrt{2}} W_{\mu}^{+} \overline{\left(\begin{array}{lll}
u & c & t
\end{array}\right)} \gamma^{\mu}\left(1-\gamma^{5}\right) V_{\mathrm{CKM}}\left(\begin{array}{l}
d \\
s \\
b
\end{array}\right)+\text { h.c. }
$$

where $V_{\mathrm{CKM}}$ is the Cabibbo-Kobayashi-Maskawa (CKM) matrix:

$$
V_{\mathrm{CKM}}=\left(\begin{array}{ccc}
V_{u d} & V_{u s} & V_{u b} \\
V_{c d} & V_{c s} & V_{c b} \\
V_{t d} & V_{t s} & V_{t b}
\end{array}\right)
$$

In the SM context $V_{\mathrm{CKM}}$ should be unitary. Any deviation from its unitarity can be a signal of new physics beyond the SM (BSM).

At present, the determinations of $\left|V_{u s}\right|$ and $\left|V_{u d}\right|$ have reached high enough precision to test with high accuracy the unitarity of the first row in CKM matrix (1.2):

$$
\left|V_{u d}\right|^{2}+\left|V_{u s}\right|^{2}+\left|V_{u b}\right|^{2}=1
$$

Namely, precision experimental data on kaon decays, in combination with the latest lattice QCD calculations of the decay constants and form-factors, provide accurate information about $\left|V_{u s}\right|$. On the other hand, recent calculations of short-distance radiative corrections in $\beta$-decays substantially improved the determination of $\left|V_{u d}\right|$. Since the contribution of $\left|V_{u b}\right| \simeq 0.004$ is very small and actually negligible, the test of the sum rule in eq. (1.3) is practically equivalent to a Cabibbo universality check.

In our previous paper ref. [1] it was pointed out that there is a significant (about $4 \sigma$ ) anomaly in the first row unitarity (1.3), after using three types of independent determinations of $\left|V_{u s}\right|$ and $\left|V_{u d}\right|$, which were dubbed as determinations of type A, B and C. Specifically, determination A corresponds to the direct determination of $\left|V_{u s}\right|$ from the kaon semileptonic $(K \ell 3)$ decays, B comes from the determination of the ratio $\left|V_{u s} / V_{u d}\right|$ obtained from charged kaon leptonic $(K \mu 2)$ decays by comparing them with pion leptonic decays, and $\mathrm{C}$ corresponds to the direct determination of $\left|V_{u d}\right|$ from superallowed $0^{+}-0^{+}$ nuclear transitions by employing the value of the Fermi constant obtained from the muon decay, $G_{F}=G_{\mu}$.

For explaining this anomaly we proposed two possible BSM scenarios. One is related to a new physics in the lepton sector. Namely, we considered the horizontal gauge symmetry $\mathrm{SU}(3)_{\ell} \times \mathrm{SU}(3)_{e}$ between the lepton families, with $\mathrm{SU}(3)_{\ell}$ acting between $\mathrm{LH}$ states $\ell_{L i}=$ $\left(\nu_{L}, e_{L}\right)_{i}$ and $\mathrm{SU}(3)_{e}$ acting between $\mathrm{RH}$ ones $e_{R i}$. We have shown that flavour changing 
gauge bosons of $\mathrm{SU}(3)_{\ell}$ induce an effective operator which contributes to the muon decay in positive interference with the SM contribution ( $W$-boson exchange). In this way, the muon decay constant $G_{\mu}$ becomes different from the Fermi constant $G_{F}: G_{\mu}=G_{F}\left(1+\delta_{\mu}\right)$ with $\delta_{\mu}=\left(v_{w} / v_{F}\right)^{2}$, where $v_{w}$ and $v_{F}$ respectively are the electroweak and horizontal symmetry breaking scales. Since the values of $\left|V_{u s}\right|$ and $\left|V_{u d}\right|$ are normally extracted by assuming $G_{F}=G_{\mu}$, in this scenario they are shifted by a factor $1+\delta_{\mu}$ while their ratio is not affected. CKM unitarity is recovered with $\delta_{\mu} \simeq 7 \times 10^{-4}$ which corresponds to a horizontal breaking scale of about $6 \mathrm{TeV}$. Interesting point is that such a low mass scale for the horizontal gauge bosons is not in conflict with the stringent experimental limits on the lepton flavour changing processes as $\mu \rightarrow 3 e, \tau \rightarrow 3 \mu$ etc. [1]. The breaking scale of $\mathrm{SU}(3)_{e}$ symmetry of the RH leptons can also be as small as few $\mathrm{TeV}$ without contradicting experimental limits [2].

Another (more straightforward) possibility discussed in ref. [1] is to introduce extra vector-like quarks. ${ }^{1}$ In particular, with extra isosinglet quarks of down-type $b^{\prime}$ or up-type $t^{\prime}$ one can settle the CKM unitarity problem which results from the determination of $V_{u d}$ from superallowed beta decays (C) and $V_{u s}$ from kaon decays (A and B), whereas by employing the extra quarks forming the weak isodoublet $\left(t^{\prime}, b^{\prime}\right)$ all the tensions between the independent determinations $\mathrm{A}, \mathrm{B}$ and $\mathrm{C}$ can in principle be explained.

However, large mixings with SM families induce flavour changing phenomena which can be in potential conflict with stringent experimental limits. In this work we give a detailed study of the effects on relevant flavour changing processes and electroweak observables and constrain the parameter space for each scenario (extra weak isosinglets of up-type or down-type or weak isodoublets).

As we will show, there still remains some available parameter space which can satisfy these stringent constraints but it is very limited and can be excluded with future experimental data. In particular, it can be excluded if the limits on masses of extra vector-like species will increase up to $3 \mathrm{TeV}$ or so or the limits on some relevant flavour changing phenomena or $Z$ boson physics will further strengthen. Therefore, all these scenarios can be falsified in close future.

The paper is organized as follows. Since after ref. [1] some new data appeared, in section 2 we update the analysis of the CKM first row anomalies. As we will show, although numbers have changed, the anomalies are still there. In section 3 we discuss the generalities about the role of different types of vectorlike quarks in fixing the problem. In sections 4 and 5 we analyze separately the scenarios with extra weak isosinglet quarks of down-type $\left(b^{\prime}\right)$ and up-type $\left(t^{\prime}\right)$, by providing a detailed study of flavour changing phenomena induced in this scenarios and determining the available parameter space. In section 6 we perform the analysis in the case of additional extra weak isodoublet $\left(t^{\prime}, b^{\prime}\right)$. In section 6.5 we discuss some combinations in case more families are introduced. At the end, in section 7 we give our conclusions.

\footnotetext{
${ }^{1}$ After ref. [1] the problem of the CKM unitarity anomaly was addressed with different approaches in several subsequent papers [3-16].
} 


\section{Present situation in the determination of $\left|V_{u s}\right|$ and $\left|V_{u d}\right|$}

As already stated, the precision of recent determinations of $\left|V_{u s}\right|$ and $\left|V_{u d}\right|$ allows to test the first row unitarity (1.3) of CKM matrix. Deviation from unitarity can be parameterized as

$$
\left|V_{u d}\right|^{2}+\left|V_{u s}\right|^{2}+\left|V_{u b}\right|^{2}=1-\delta_{\mathrm{CKM}}
$$

Hence, the value $\delta_{\mathrm{CKM}}$ shows the measure of the unitarity deficit.

The element $\left|V_{u s}\right|$ can be directly determined from semileptonic $K \ell 3$ decays $\left(K_{L} \mu 3\right.$, $K_{L} e 3, K^{ \pm} e 3$, etc.) which imply [17]:

$$
f_{+}(0)\left|V_{u s}\right|=0.21654 \pm 0.00041
$$

where $f_{+}(0)$ is the vector form factor at zero momentum transfer which can be computed in the lattice QCD simulations. The average of 4-flavor computations reported by FLAG 2019 is $f_{+}(0)=0.9706(27)$ [18]. We combine it with the latest 4-flavor result $f_{+}(0)=$ 0.9696(19) [19] (which was not included in FLAG 2019 [18]) getting $f_{+}(0)=0.9699(15)$. In this way, from eq. (2.2) we obtain the value of $\left|V_{u s}\right|$ (determination $\mathrm{A}$ in the following) as:

$$
\mathrm{A}: \quad\left|V_{u s}\right|_{\mathrm{A}}=0.22326(55)
$$

An independent information (determination B in the following) stems from the ratio of the kaon and pion leptonic decay rates $K \rightarrow \mu \nu(\gamma)$ and $\pi \rightarrow \mu \nu(\gamma)$ which implies [20]:

$$
\left|V_{u s} / V_{u d}\right| \times\left(f_{K^{ \pm}} / f_{\pi^{ \pm}}\right)=0.27599 \pm 0.00038
$$

Then, by employing the 4-flavour average for the decay constants ratio $f_{K^{ \pm}} / f_{\pi^{ \pm}}=1.1932(19)$ reported in FLAG 2019 [18], we obtain:

$$
\text { B : } \quad\left|V_{u s}\right| /\left|V_{u d}\right|=0.23130(49)
$$

As regards the element $\left|V_{u d}\right|$, its most precise determination is obtained from superallowed $0^{+}-0^{+}$nuclear $\beta$-decays, which are pure Fermi transitions sensitive only to the vector coupling constant $G_{V}=G_{F}\left|V_{u d}\right|$. The master formula reads [21, 22]:

$$
\left|V_{u d}\right|^{2}=\frac{K}{2 G_{F}^{2} \mathcal{F} t\left(1+\Delta_{R}\right)}=\frac{0.97142(58)}{1+\Delta_{R}}
$$

where $K=2 \pi^{3} \ln 2 / m_{e}^{5}=8120.2765(3) \times 10^{-10} \mathrm{~s} / \mathrm{GeV}^{4}, G_{F}=G_{\mu}=1.1663787(6) \times$ $10^{-5} \mathrm{GeV}^{-2}$ is the Fermi constant determined from the muon decay [24] and the nucleus independent value $\mathcal{F} t=3072.24(1.85) \mathrm{s}$ is derived from $f t$-values of 15 best determined superallowed $0^{+}-0^{+}$nuclear transitions by absorbing in the latter all transition-dependent (so called outer) corrections. This value has been very recently updated in ref. [22]. In particular, while the $\mathcal{F t}$ central value is almost unchanged, the uncertainty attributed to the theoretical corrections has increased. ${ }^{2}$ The second source of uncertainty in $V_{u d}$ is

\footnotetext{
${ }^{2}$ In our previous work [1] we used $\mathcal{F} t=3072.07(72) \mathrm{s}$ [23], but this value was recently upadated in ref. [22].
} 
related to the transition independent short-distance (so called inner) radiative correction $\Delta_{R}$ which in 2006 was computed by Marciano and Sirlin obtaining $\Delta_{R}=0.02361(38)$ [25].

However, a recent calculation with improved hadronic uncertainties brought to a drastically different value $\Delta_{R}=0.02467(22)$ [26]. A more conservative approach of ref. [27] gives a slightly lower result with relatively larger uncertainty, $\Delta_{R}=0.02426(32)$. For our analysis we decided to use the average of these two results, $\Delta_{R}=0.02454(18){ }^{3}$ Then, using this average, from eq. (2.6) we get the value of $\left|V_{u d}\right|$ :

$$
0^{+}-0^{+}: \quad\left|V_{u d}\right|=0.97373(29)_{\mathcal{F} t}(9)_{\Delta_{R}}=0.97373(31)
$$

The value of $\left|V_{u d}\right|$ can be extracted also from the free neutron $\beta$-decay:

$$
\left|V_{u d}\right|^{2}=\frac{K / \ln 2}{G_{F}^{2} \mathcal{F}_{n} \tau_{n}\left(1+3 g_{A}^{2}\right)\left(1+\Delta_{R}\right)}=\frac{5024.46(60) \mathrm{s}}{\tau_{n}\left(1+3 g_{A}^{2}\right)\left(1+\Delta_{R}\right)}
$$

where $\mathcal{F}_{n}=f_{n}\left(1+\delta_{R}^{\prime}\right)$ is the neutron $f$-value $f_{n}=1.6887(2)$ corrected by the long-distance QED correction $\delta_{R}^{\prime}=0.014902(2)$ [31]. It is somewhat less precise due to limited accuracy in the experimental determination of the neutron lifetime $\tau_{n}$ and the axial coupling constant $g_{A}=G_{A} / G_{V}$. By combining the recent determination of axial coupling $g_{A}=1.27625(50)$ and the "bottle" lifetime $\tau_{n}^{\text {bottle }}=879.4(0.6) \mathrm{s}$ as in ref. [1], using the average $\Delta_{R}=$ 0.02454(18), we get:

$$
\text { free neutron: } \quad\left|V_{u d}\right|=0.97333(33)_{\tau_{n}}(32)_{g_{A}}(9)_{\Delta_{R}}(6)_{f_{n}}=0.97333(47)
$$

(Interestingly, by comparing the determinations of $\left|V_{u d}\right|$ from free neutron decays and superallowed $0^{+}-0^{+}$decays, the factor $1+\Delta_{R}$ cancels out and one obtains an accurate determination of the neutron lifetime $\tau_{n}=5172.0(1.1) /\left(1+3 g_{A}^{2}\right)=878.7(0.6)[33]$ which well agrees with $\tau_{n}^{\text {bottle }}$ but is in strong tension with $\tau_{n}^{\text {beam }}=888.0(2.0) \mathrm{s} \mathrm{[32].)}$

By averaging the results from superallowed beta decays and free neutron decay (2.7), (2.9) we get the value of $\left|V_{u d}\right|$ (determination $\mathrm{C}$ in the following):

$$
\mathrm{C}: \quad\left|V_{u d}\right|=0.97362(26)
$$

The measurements of $\pi^{+} \rightarrow \pi^{0} e^{+} \nu$ branching ratio by PIBETA experiment [34] lead to the independent result $\left|V_{u d}\right|=0.9728(30)$ which however has about 10 and 6 times larger uncertainties as compared to determinations (2.7) and (2.9). Therefore, we take in consideration determination $\mathrm{C}$ obtained from superallowed $0^{+}-0^{+}$transitions and free neutron decay.

The tensions between determinations $\mathrm{A}, \mathrm{B}$ and $\mathrm{C}$ are shown in figure 1, which presents the fit of the values $(2.3),(2.5),(2.10)$, with $V_{u s}$ and $V_{u d}$ considered as independent parameters, without imposing unitarity. The unitarity condition (1.3) is shown with the black

\footnotetext{
${ }^{3}$ Other more recent studies confirmed the shift of the value of $\Delta_{R}^{V}$ [28-30]. By including these results, the discrepancies in the first row of the CKM matrix would increase, so we are more conservative with our choice.

${ }^{4}$ There is an apparent tension $(4 \sigma)$ between the neutron lifetime measurements using the bottle and beam experimental methods, $\tau_{n}^{\text {bottle }}=879.4(0.6) \mathrm{s}$ and $\tau_{n}^{\text {beam }}=888.0(2.0) \mathrm{s}$, origin of which requires more profound understanding and perhaps some new physics [32].
} 


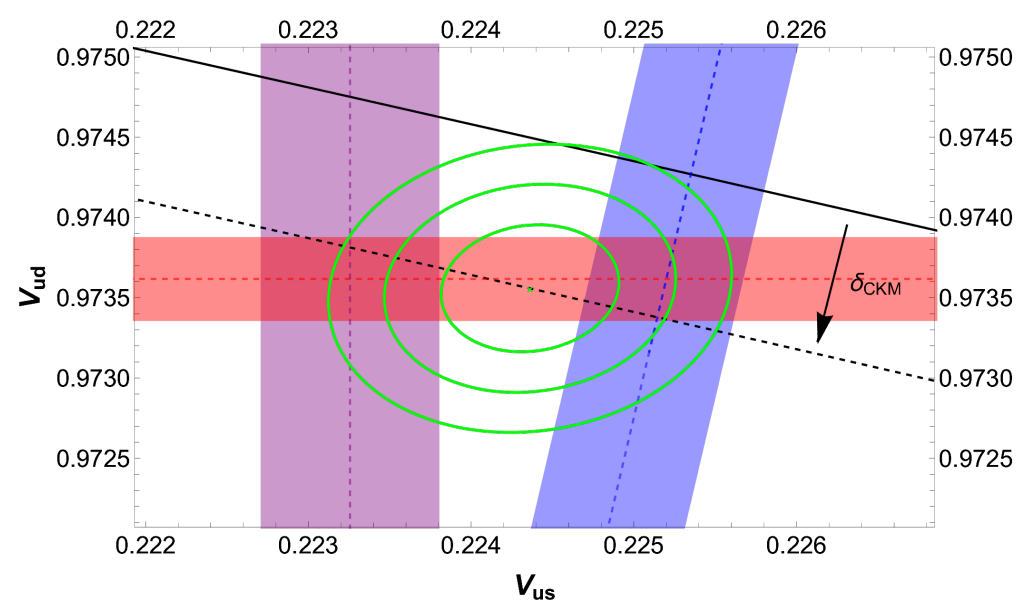

Figure 1. The purple, blue and red bands correspond respectively to the values of $\left|V_{u s}\right|$ from eq. (2.3), $\left|V_{u s} / V_{u d}\right|$ from eq. (2.5) and $\left|V_{u d}\right|=0.97362(26)$ from eq. (2.10). The best fit point, $1 \sigma$, $2 \sigma$ and $3 \sigma$ coverage probability contours are shown (green cross and green circles) for $V_{u s}$ and $V_{u d}$ considered as independent parameters, without imposing unitarity. The black curve corresponds to the unitarity condition (1.3). The dashed black curve corresponds to eq. (2.1) with the deficit of unitarity $\delta_{\mathrm{CKM}}=1.8 \times 10^{-3}$.

continuous line. The best fit (minimum $\chi^{2}$ ) corresponds to

$$
\left|V_{u s}\right|=0.22436(36) \quad\left|V_{u d}\right|=0.97356(26)
$$

The unitarity curve is $3 \sigma$ away. The $\chi^{2}$ value is rather large, $\chi^{2}=7.1$, due to the tension between the two determinations $\mathrm{A}$ and $\mathrm{B}$ from kaon decays. Using eq. (2.1) for fitting the data, the deficit of CKM unitarity results

$$
\delta_{\mathrm{CKM}}=1.8(5) \times 10^{-3}
$$

The tensions can be manifested also in another way. We can take $\left|V_{u s}\right|_{\mathrm{A}}=0.22326(55)$ from the direct determination $\mathrm{A}$ while $\mathrm{B}$ and $\mathrm{C}$ can be also translated in $\left|V_{u s}\right|$ determinations by imposing the unitarity condition (1.3). Namely, the value of $\left|V_{u s}\right|$ obtained in this way from eq. (2.5) is:

$$
\left|V_{u s}\right|_{\mathrm{B}}=0.22535(45)
$$

which is compatible also with a theoretical result $\left|V_{u s}\right|=0.22567(42)$ from $K \mu 2$ decays obtained in ref. [35].

From determination C (2.10) we get instead:

$$
\left|V_{u s}\right|_{\mathrm{C}}=0.2282(11)
$$

For completeness, in table 1 we show the values of $\left|V_{u d}\right|$ and respective "unitarity" values of $\left|V_{u s}\right|$ corresponding to the choices of $\Delta_{R}$ as reported in original refs. [25-27], indicated as $\mathrm{C}_{0}, \mathrm{C}_{1}$ and $\mathrm{C}_{2}$. 


\begin{tabular}{|lccc|}
\hline Determination & $\Delta_{R}$ & $\left|V_{u d}\right|$ & $\left|V_{u s}\right|$ \\
\hline $\mathrm{C}_{0}[25]$ & $0.02361(38)$ & $0.97420(21)$ & $0.2257(9)$ \\
$\mathrm{C}_{1}[26]$ & $0.02467(22)$ & $0.97355(27)$ & $0.2284(11)$ \\
$\mathrm{C}_{2}[27]$ & $0.02426(32)$ & $0.97375(29)$ & $0.2276(12)$ \\
$\mathrm{C}$ (our choice) & $0.02454(18)$ & $0.97362(26)$ & $0.2282(11)$ \\
\hline
\end{tabular}

Table 1. Values of $\Delta_{R}$ reported in original references [26, 27], respectively labeled as $\mathrm{C}_{1}$ and $\mathrm{C}_{2}$, and corresponding values of $\left|V_{u d}\right|$ obtained by averaging eqs. (2.6) and (2.8). Values of $\left|V_{u s}\right|$ are obtained assuming unitarity (1.3). C represents our average (see text). $\mathrm{C}_{0}$ represents the value quoted by Particle Data Group (PDG) review 2018 [36].

\begin{tabular}{|lccc|}
\hline & $\mathrm{A}:\left|V_{u s}\right|=0.22326(55)$ & $\mathrm{B}:\left|V_{u s}\right|=0.23130(49)\left|V_{u d}\right|$ & Average $^{*}$ \\
\hline $\mathrm{C}_{1}:\left|V_{u d}\right|=0.97355(27)$ & $2.3 \cdot 10^{-3}$ & $1.5 \cdot 10^{-3}$ & $1.8 \cdot 10^{-3}$ \\
$\mathrm{C}_{2}:\left|V_{u d}\right|=0.97375(29)$ & $2.0 \cdot 10^{-3}$ & $1.1 \cdot 10^{-3}$ & $1.5 \cdot 10^{-3}$ \\
$\mathrm{C}:\left|V_{u d}\right|=0.97362(26)$ & $2.2 \cdot 10^{-3}$ & $1.3 \cdot 10^{-3}$ & $1.7 \cdot 10^{-3}$ \\
\hline
\end{tabular}

*Average of the values of $V_{u s}$ given in the columns A and B.

Table 2. Values of $\delta_{\mathrm{CKM}}$ obtained for different choices of the values of $\left|V_{u s}\right|$ and $\left|V_{u d}\right|$.

Figure 2 displays the values $\left|V_{u s}\right|_{\mathrm{A}}(2.3),\left|V_{u s}\right|_{\mathrm{B}}$ (2.13) and $\left|V_{u s}\right|_{\mathrm{C}}(2.14)$ with corresponding error bars (shaded areas). Between determinations obtained from kaon decays, $\mathrm{A}$ and $\mathrm{B}$, there is about $3 \sigma$ tension, which maybe could disappear with more accurate lattice simulations. ${ }^{5}$ Therefore, we conservatively take a democratic average of $\left|V_{u s}\right|_{\mathrm{A}}$ and $\left|V_{u s}\right|_{\mathrm{B}}$ without reducing error bars (with the uncertainty taken as arithmetical average of two uncertainties):

$$
\overline{\mathrm{A}+\mathrm{B}}: \quad\left|V_{u s}\right|=0.22451(50)
$$

We see that there is about $4 \sigma$ tension between determination $\mathrm{A}$ and $\mathrm{C}$ and $2.3 \sigma$ tension between $\mathrm{B}$ and $\mathrm{C}$. The discrepancy between the conservative averages $\overline{\mathrm{A}+\mathrm{B}}$ and $\mathrm{C}$ results in $3 \sigma$. Let us notice that if we try to fit the incompatible determinations of $V_{u s} A(2.3)$, $B$ (2.13), $C$ (2.14), we would get the average value $\left|V_{u s}\right|=0.22482$ but with a ugly large $\chi^{2}$ value, $\chi^{2}=18$.

In table 2 we show the landscape of possible values of the unitarity deficit $\delta_{\mathrm{CKM}}$. We see that depending on the choice of the data this value spans from about $10^{-3}$ to about $2 \cdot 10^{-3}$.

In conclusion, the CKM unitarity condition with three families (1.3) is not really consistent with the present determinations of $\left|V_{u s}\right|$ and $\left|V_{u d}\right|$. An immediate solution would be to introduce a fourth sequential family $\left(t^{\prime}, b^{\prime}\right)$, analogous to the three SM families, with the LH components forming weak isodoublets and $\mathrm{RH}$ components being isosinglets. Then

\footnotetext{
${ }^{5}$ These determinations obtained from 3 -flavor lattice computations [37] were in fact compatible because of larger error-bars, see figure 2 .
} 


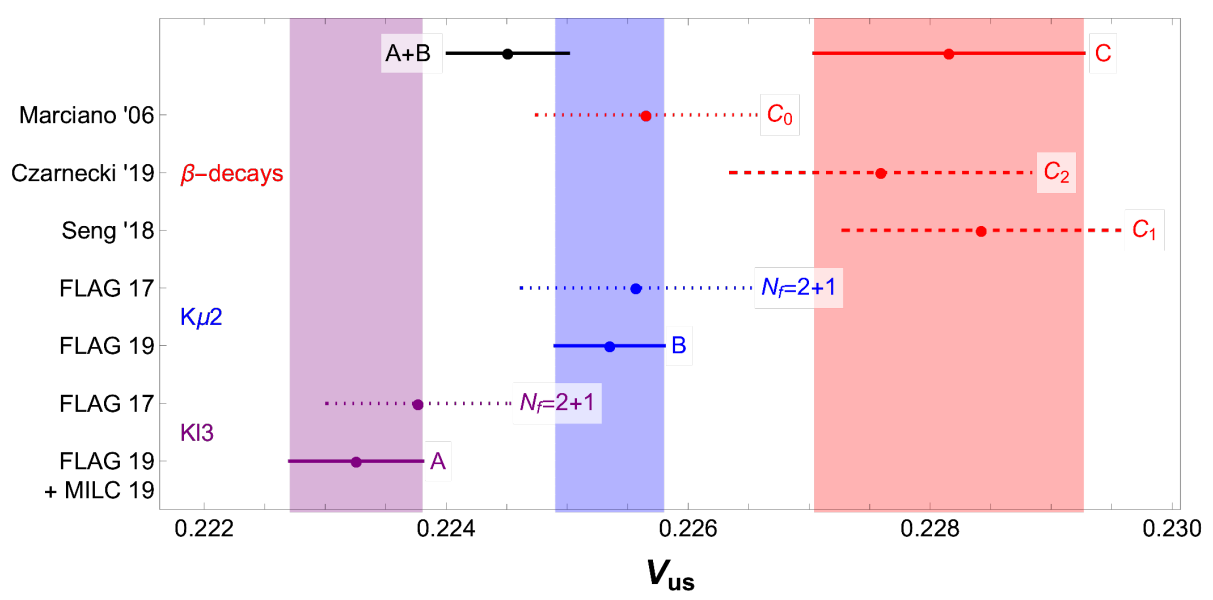

Figure 2. Shaded areas show the values of $\left|V_{u s}\right|$ obtained from determinations A (2.3), B (2.5) and $\mathrm{C}$ (2.10) by assuming CKM unitarity (1.3), while the black line corresponds to the democratic average of $\mathrm{A}$ and $\mathrm{B}$ (2.15) (see text). $\mathrm{C}_{1}, \mathrm{C}_{2}$ are the values of $\left|V_{u s}\right|$ obtained from the values of $\Delta_{R}$ reported by original references, as listed in table 1 . For comparison we also show the values of $\left|V_{u s}\right|$ obtained using 3 flavours lattice QCD simulations as reported in FLAG 17 [37] and adopted in Particle Data Group 2018. These determinations have practically no tension with the old determination $\mathrm{C}_{0}[25]$. Hence, this picture demonstrate that the CKM tensions in fact emerged due to improved precision of 4 -flavours computations $[18,19]$ on one side, and due to the changes in inner radiative correction $\Delta_{R}[26,27]$ on the other side.

the $3 \times 3$ CKM matrix (1.2) should be extended to a unitary $4 \times 4$ matrix:

$$
V_{\mathrm{CKM}}=\left(\begin{array}{cccc}
V_{u d} & V_{u s} & V_{u b} & V_{u b^{\prime}} \\
V_{c d} & V_{c s} & V_{c b} & V_{c b^{\prime}} \\
V_{t d} & V_{t s} & V_{t b} & V_{t b^{\prime}} \\
V_{t^{\prime} d} & V_{t^{\prime} s} & V_{t^{\prime} b} & V_{t^{\prime} b^{\prime}}
\end{array}\right),
$$

and correspondingly the first row unitarity condition would be modified to:

$$
\left|V_{u d}\right|^{2}+\left|V_{u s}\right|^{2}+\left|V_{u b}\right|^{2}+\left|V_{u b^{\prime}}\right|^{2}=1
$$

Comparing this equation with eq. (2.1) we see that the parameter $\delta_{\mathrm{CKM}}$ assumes the meaning of the mixing with the fourth family, $\delta_{\mathrm{CKM}}=\left|V_{u b^{\prime}}\right|^{2}$. Therefore for typical values of $\delta_{\mathrm{CKM}}$ given in table 2 we get $\left|V_{u b^{\prime}}\right| \approx 0.04$, which is comparable with $\left|V_{c b}\right|$ and an order of magnitude larger than $\left|V_{u b}\right| \approx 0.004$. It looks not very natural that the mixing of the first family with the fourth family is much stronger than its mixing with the third family, but some models admit this possibility [39]. Unfortunately, the existence of a fourth sequential family is excluded by the limits from electroweak precision data combined with the LHC data.

However, vector-like quarks can be introduced without any contradiction with SM precision tests. The LHC limits merely tell that their masses should be above $1 \mathrm{TeV}$ or so.

In the rest of this paper we discuss how the anomalies in the first row of CKM matrix can be solved by introducing extra vector-like fermions. In particular we will consider the role of weak isosinglets of down-type or up-type and weak isodoublets. 
In fact, two approaches to the problem can be considered. The incompatibility inside kaon physics may be attributed to some uncertainties which can disappear maybe soon with more precise determinations, focusing instead on the average of determinations from kaons. Then the problem consists in solving the lack of unitarity in the first row of $V_{\text {CKM }}$. The insertion of an extra vectorlike weak isosinglet, down-type or up-type, is on this line.

However, the $V_{u s}$ anomaly (discrepancy between $V_{u s}$ determinations from the kaon semileptonic and leptonic decays) can be considered seriously, ${ }^{6}$ and one has to look for a solution addressing the whole situation. As it will be shown, a weak isodoublet can in principle explain all the anomalies.

In these scenarios with extra vector-like families, the unitarity deficit $\delta_{\mathrm{CKM}}$ will be related to the mixing of the extra quarks with the SM families.

\section{The SM with extra vector-like fermions}

The Standard Model SU $(3) \times \mathrm{SU}(2) \times \mathrm{U}(1)$ contains, by definition, three chiral families of fermions, the LH quarks $q_{L i}=\left(u_{L}, d_{L}\right)_{i}$ and leptons $\ell_{L i}=\left(\nu_{L}, e_{L}\right)_{i}$ transforming as $\mathrm{SU}(2)$ isodoublets and the RH components $u_{R i}, d_{R i}, e_{R i}$ being isosinglets, with $i=1,2,3$ being the family index. This set of fermions is free of gauge anomalies. Attractive property of the $\mathrm{SM}$ is that these fermions can acquire masses only via the Yukawa couplings with Higgs doublet $\varphi$ :

$$
\mathcal{L}_{\mathrm{Yuk}}^{\mathrm{SM}}=Y_{u}^{i j} \tilde{\varphi} \overline{q_{L i}} u_{R j}+Y_{d}^{i j} \varphi \overline{q_{L i}} d_{R j}+Y_{e}^{i j} \varphi \overline{\ell_{L i}} e_{R j}+\text { h.c. }
$$

where $Y_{u, d, e}^{i j}$ are the Yukawa constant matrices, and $\tilde{\varphi}=i \tau_{2} \varphi^{*}$. The known species of quarks and leptons are eigenstates of mass matrices $M_{u, d, e}=Y_{u, d, e} v_{w}$ where $\left\langle\varphi^{0}\right\rangle=v_{w}$ is the Higgs VEV. In other words, in the SM the quark and lepton masses are induced only after the electroweak symmetry breaking, and their values are proportional to the electroweak scale $v_{w}$.

The quark mass matrices can be diagonalized by bi-unitary transformations

$$
V_{L u}^{\dagger} M_{u} V_{R u}=\widetilde{M}_{u}=\operatorname{diag}\left(m_{u}, m_{c}, m_{t}\right), \quad V_{L d}^{\dagger} M_{d} V_{R d}=\widetilde{M}_{d}=\operatorname{diag}\left(m_{d}, m_{s}, m_{b}\right)
$$

and the weak eigenstates in terms of mass eigenstates are:

$$
\left(\begin{array}{c}
u_{L 1} \\
u_{L 2} \\
u_{L 3}
\end{array}\right)=V_{L u}\left(\begin{array}{c}
u_{L} \\
c_{L} \\
t_{L}
\end{array}\right) ; \quad \quad\left(\begin{array}{c}
d_{L 1} \\
d_{L 2} \\
d_{L 3}
\end{array}\right)=V_{L d}\left(\begin{array}{c}
d_{L} \\
s_{L} \\
b_{L}
\end{array}\right)
$$

The CKM mixing matrix in $W$ boson charged current couplings (1.1) emerges as a combination of the 'left' unitary transformations, $V_{\mathrm{CKM}}=V_{L u}^{\dagger} V_{L d}$, and thus it should be unitary. As for the 'right' matrices $V_{R u}$ and $V_{R d}$, in the SM frames they have no physical significance. Without loss of generality, one can choose a fermion basis in which one

\footnotetext{
${ }^{6}$ In fact, a recent high precision determination of $K \ell 3$ radiative corretions [38] indicates that the SM electroweak effects are not large enough to account for $V_{u s}$ anomaly.
} 
of the Yukawa matrices $Y_{u}$ or $Y_{d}$ is diagonal in which cases we would have respectively $V_{\mathrm{CKM}}=V_{L d}$ or $V_{\mathrm{CKM}}=V_{L u}^{\dagger}$.

The SM exhibits a remarkable feature of natural suppression of flavor-changing neutral currents (FCNC) [40-42]: no flavor mixing emerges in neutral currents coupled to $Z$ boson and Higgs boson. In particular, this means that $Z$ boson tree level couplings with the fermion mass eigenstates remain diagonal after rotations (3.3). On the other hand, the Yukawa matrices $Y_{u, d, e}$ and mass matrices $M_{u, d, e}$ are proportional and thus by transformations (3.2) they are diagonalized simultaneously, so that the Yukawa couplings of the Higgs boson $H$ with the fermion mass eigenstates are diagonal. Hence, all FCNC phenomena are suppressed at tree level and emerge exclusively from radiative corrections. At present, the majority of experimental data on flavor changing and $\mathrm{CP}$ violating processes are in good agreement with the SM predictions.

Clearly, in the SM framework the unitarity of the CKM matrix as well as the natural flavor conservation in neutral currents are direct consequences following from the fact that the three families are in identical representations of $\mathrm{SU}(3) \times \mathrm{SU}(2) \times \mathrm{U}(1)$.

However, in addition to three chiral families of quarks and leptons, there can exist extra vector-like species, with the $\mathrm{LH}$ and $\mathrm{RH}$ in the same representations of the SM. In particular, one can consider the extra fermion species in the same representations of $\mathrm{SU}(3) \times \mathrm{SU}(2) \times \mathrm{U}(1)$ as standard quarks and leptons, namely in the form of weak isosinglets of down quark type $D_{L, R}$, up quark type $U_{L, R}$ and charged lepton type $E_{L, R}$, and weak isodoublets $Q_{L, R}=(\mathcal{U}, \mathcal{D})_{L, R}$ and $L_{L, R}=(\mathcal{N}, \mathcal{E})_{L, R}$ of quark and lepton types. ${ }^{7}$ (Extra vector-like fermions can be introduced also in other representations as e.g. $\mathrm{SU}(2)$ isotriplets which can contain quark or lepton type fragments but also some fragments with exotic electric charges but here we do not address these cases.) The mass terms of these species are not protected by the SM gauge symmetries and hence their masses can be (or must be) considerably larger than the electroweak scale.

In the following we shall concentrate on the quark sector. Namely, we consider a theory which, besides the three chiral families of standard quarks $u_{R i}, d_{R i}$ and $q_{L i}=\left(u_{L}, d_{L}\right)_{i}$ $(i=1,2,3)$, includes some extra vector-like quark species $U_{L, R}, D_{L, R}$ and $Q_{L, R}=(\mathcal{U}, \mathcal{D})_{L, R}$ which in principle can be introduced in different amounts. Therefore, along with the standard Yukawa terms for the three chiral families: ${ }^{8}$

$$
\mathcal{L}_{\text {Yuk }}=Y_{u} \tilde{\varphi} \overline{q_{L}} u_{R}+Y_{d} \varphi \overline{q_{L}} d_{R}+\text { h.c. }
$$

the most general Lagrangian of this system must include the mixed Yukawa terms between the standard and extra species:

$$
\mathcal{L}_{\text {Yuk }}^{\operatorname{mix}}=h_{U} \tilde{\varphi} \overline{q_{L}} U_{R}+h_{D} \varphi \overline{q_{L}} D_{R}+h_{\mathcal{U}} \tilde{\varphi} \overline{Q_{L}} u_{R}+h_{\mathcal{D}} \varphi \overline{Q_{L}} d_{R}+\text { h.c. }
$$

\footnotetext{
${ }^{7}$ Such vector-like species are predicted in some extensions of the Standard Model. For example, $D$ and $L$ type species emerge (per each family) in the context of minimal $E_{6}[43,44]$ or SU(6) [45] grand unifications. In addition, the specifics of the latter model in which Higgs emerges as pseudo-Goldstone particle, requires at least one copy of $Q, U$ and $E$ type species for inducing the fermion masses and in particular the top quark mass $[46,47]$.

${ }^{8}$ Hereafter indices $i=1,2,3$ of normal families as well as indices of extra species are suppressed.
} 
and the mass terms

$$
\mathcal{L}_{\text {mass }}=M_{U} \overline{U_{L}} U_{R}+M_{D} \overline{D_{L}} D_{R}+M_{Q} \overline{Q_{L}} Q_{R}+\mu_{u} \overline{U_{L}} u_{R}+\mu_{d} \overline{D_{L}} d_{R}+\mu_{q} \overline{q_{L}} Q_{R}+\text { h.c. }
$$

where $h_{U, D}$ and $h_{u, d}$ in the Yukawa terms (3.5) and $M_{U, D, Q}$ and $\mu_{u, d, q}$ in mass terms (3.6) are the matrices of proper dimensions depending on the amounts of extra species. One could introduce also the Yukawa couplings between extra species:

$$
\mathcal{L}_{\text {Yuk }}^{\text {extra }}=\lambda_{U} \tilde{\varphi} \overline{Q_{L}} U_{R}+\lambda_{\mathcal{U}} \tilde{\varphi} \overline{Q_{R}} U_{L}+\lambda_{D} \varphi \overline{Q_{L}} D_{R}+\lambda_{\mathcal{D}} \varphi \overline{Q_{R}} D_{L}+\text { h.c. }
$$

However, they play no relevant role in further discussions and for simplicity we neglect them.

The vector-like quarks are key players in models with horizontal inter-family symmetries [48-55], in some models of the axion [56-60] as well as in axionless (Nelson-Barr type) models for solving the strong $\mathrm{CP}$ problem via spontaneous $\mathrm{CP}$ or $\mathrm{P}$ violation [61-65]. In these models the values $M_{U, D, Q}$ and $\mu_{u, d, q}$ in mass terms (3.6) are related to the breaking scales of respective symmetries, and in some models e.g. [63, 65, 66] they can be as low as few $\mathrm{TeV}$.

Interestingly, some of these symmetries (e.g. flavor symmetry or Peccei-Quinn symmetry) may forbid the direct Yukawa terms (3.4) but allow the mixed ones (3.5) while mass terms $\mu$ and $M$ in (3.5) can be originated from some physical scales. ${ }^{9}$ Nevertheless, despite that the original constants $Y_{u, d}$ in (3.4) are vanishing, the SM Yukawa terms (3.1) for normal fermions will be induced after integrating out the heavy states. In particular, provided that mixing mass terms $\mu_{u, d, q}$ are smaller than $M_{U, D, Q}$, we obtain

$$
Y_{u} \simeq h_{U} M_{U}^{-1} \mu_{u}+\mu_{q} M_{Q}^{-1} h_{\mathcal{U}}, \quad Y_{d} \simeq h_{D} M_{D}^{-1} \mu_{d}+\mu_{q} M_{Q}^{-1} h_{\mathcal{D}}
$$

In other words, the non-zero quark masses are induced via the mixings with the extra vector-like species. Such a scenario known as 'universal' seesaw mechanism [48, 67-69] is commonly used in predictive model building for fermion masses and mixings as e.g. [48, 6976]. In the context of supersymmetric models with flavor symmetry this mechanism can give a natural realization of the minimal flavor violation scenario via the alignment of soft supersymmetry breaking terms with the Yukawa terms [77-79].

In the following we are not interested in the model details and in possible dynamical effects of the underlying symmetries broken at higher scales, but only in the effects of the mixing between the three normal (chiral) and extra (vector-like) quarks. Therefore, we can conveniently redefine the fermion basis. Namely, the species $u_{R}$ and $U_{R}, d_{R}$ and $D_{R}$, and $q_{L}$ and $Q_{L}$, are in the identical representations of $\mathrm{SU}(3) \times \mathrm{SU}(2) \times \mathrm{U}(1)$. Thus, by redefining these species, one can eliminate mixed mass terms $\mu_{u}, \mu_{d}$ and $\mu_{q}$ in (3.6) by 'absorbing' them respectively in the mass terms $M_{U}, M_{D}$ and $M_{Q}$ (this means that e.g. from $3+n$ RH species with quantum numbers of $d_{R}$ we can always select $n$ their combinations which

\footnotetext{
${ }^{9}$ E.g. in the 'seesaw' model of ref. [48] the values of $\mu_{u, d}$ and $M_{U, D}$ are respectively determined by the breaking scales of left-right symmetry and family symmetry, i.e. by the VEV of the 'right' Higgs doublet and VEVs of flavon scalars which break the horizontal symmetry.
} 
'marry' $n$ species of LH fermions $\mathcal{D}_{L}$ via mass terms while the remaining 3 combinations have no mass terms). In addition, without losing generality, the 'heavy' mass matrices $M_{U}$, $M_{D}$ and $M_{Q}$ can be taken to be diagonal and real.

In this basis the total mass matrices of up type $(u, U, \mathcal{U})_{L, R}$ and down type $(d, D, \mathcal{D})_{L, R}$ quarks, after substituting the Higgs $\operatorname{VEV}\langle\phi\rangle=v_{w}$, read:

$$
\mathcal{M}_{\text {up }}=\left(\begin{array}{ccc}
Y_{u} v_{w} & h_{U} v_{w} & 0 \\
0 & M_{U} & 0 \\
h_{\mathcal{U}} v_{w} & 0 & M_{Q}
\end{array}\right), \quad \mathcal{M}_{\text {down }}=\left(\begin{array}{ccc}
Y_{d} v_{w} & h_{D} v_{w} & 0 \\
0 & M_{D} & 0 \\
h_{\mathcal{D}} v_{w} & 0 & M_{Q}
\end{array}\right)
$$

where the blocks $Y_{u, d}$ are matrices of dimensions $3 \times 3$. Assuming that the numbers of extra species $U, D$ and $Q$ are respectively $p, n$ and $m$, then blocks $M_{U}, M_{D}$ and $M_{Q}$ should be correspondingly of dimensions $p \times p, n \times n$ and $m \times m$. Thus, $\mathcal{M}_{\text {up }}$ and $\mathcal{M}_{\text {down }}$ respectively are $(3+p+m) \times(3+p+m)$ and $(3+n+m) \times(3+n+m)$ matrices.

The mass matrices (3.9) can be brought to the diagonal forms via bi-unitary transformations $\left(\mathcal{V}_{\text {up }}^{L}\right)^{\dagger} \mathcal{M}_{\text {up }} \mathcal{V}_{\text {up }}^{R}=\widetilde{\mathcal{M}}_{\text {up }}$ and $\left(\mathcal{V}_{\text {down }}^{L}\right)^{\dagger} \mathcal{M}_{\text {down }} \mathcal{V}_{\text {down }}^{R}=\widetilde{\mathcal{M}}_{\text {down }}$. In this way, the initial states of e.g. down-type quarks are related to their physical states (mass eigenstates) as

$$
\left(\begin{array}{c}
d \\
D \\
\mathcal{D}
\end{array}\right)_{L, R}=\mathcal{V}_{\text {down }}^{L, R}\left(\begin{array}{c}
d^{\prime} \\
D^{\prime} \\
\mathcal{D}^{\prime}
\end{array}\right)_{L, R}=\left(\begin{array}{ccc}
V_{d d^{\prime}}^{L, R} & V_{d D^{\prime}}^{L, R} & V_{d \mathcal{D}^{\prime}}^{L, R} \\
V_{D d^{\prime}}^{L, R} & V_{D D^{L}, R}^{L} & V_{D \mathcal{D}^{\prime}}^{L, R} \\
V_{\mathcal{D} d^{\prime}}^{L, R} & V_{\mathcal{D} D^{\prime}}^{L, R} & V_{\mathcal{D} \mathcal{D}^{\prime}}^{L, R}
\end{array}\right)\left(\begin{array}{c}
d^{\prime} \\
D^{\prime} \\
\mathcal{D}^{\prime}
\end{array}\right)_{L, R}
$$

Here $d=\left(d_{1}, d_{2}, d_{3}\right)^{T}$ are initial states and $d^{\prime}=(d, s, b)^{T}$ are the mass eigenstates, and similarly for heavy species $D$ and $\mathcal{D}$. Analogously, unitary matrices $\mathcal{V}_{\text {up }}^{L, R}$ connect the initial up-quark type states $u, U, \mathcal{U}$ with their mass eigenstates $u^{\prime}, U^{\prime}, \mathcal{U}^{\prime}$, where $u=\left(u_{1}, u_{2}, u_{3}\right)^{T}$ and $u^{\prime}=(u, c, t)^{T}$.

Since we have $\widetilde{\mathcal{M}}_{\text {down }}^{2}=\left(\mathcal{V}_{\text {down }}^{L}\right)^{\dagger} \mathcal{M}_{\text {down }} \mathcal{M}_{\text {down }}^{\dagger} \mathcal{V}_{\text {down }}^{L}=\left(\mathcal{V}_{\text {down }}^{R}\right)^{\dagger} \mathcal{M}_{\text {down }}^{\dagger} \mathcal{M}_{\text {down }} \mathcal{V}_{\text {down }}^{R}$, unitary matrices $\mathcal{V}_{\text {down }}^{L}$ and $\mathcal{V}_{\text {down }}^{R}$ can be determined by considering the hermitian squares of $\mathcal{M}_{\text {down }}$ :

$$
\begin{aligned}
\mathcal{M}_{\text {down }} \mathcal{M}_{\text {down }}^{\dagger} & =\left(\begin{array}{ccc}
v_{w}^{2} Y_{d} Y_{d}^{\dagger}+v_{w}^{2} h_{D} h_{D}^{\dagger} & v_{w} h_{D} M_{D} & v_{w}^{2} Y_{d} h_{\mathcal{D}}^{\dagger} \\
v_{w} M_{D} h_{D}^{\dagger} & M_{D}^{2} & 0 \\
v_{w}^{2} h_{\mathcal{D}} Y_{d}^{\dagger} & 0 & M_{Q}^{2}+v_{w}^{2} h_{\mathcal{D}} h_{\mathcal{D}}^{\dagger}
\end{array}\right), \\
\mathcal{M}_{\text {down }}^{\dagger} \mathcal{M}_{\text {down }} & =\left(\begin{array}{ccc}
v_{w}^{2} Y_{d}^{\dagger} Y_{d}+v_{w}^{2} h_{\mathcal{D}}^{\dagger} h_{\mathcal{D}} & v_{w}^{2} Y_{d}^{\dagger} h_{D} & v_{w} h_{\mathcal{D}}^{\dagger} M_{Q} \\
v_{w}^{2} h_{D}^{\dagger} Y_{d} & M_{D}^{2}+v_{w}^{2} h_{D}^{\dagger} h_{D} & 0 \\
v_{w} M_{Q} h_{\mathcal{D}} & 0 & M_{Q}^{2}
\end{array}\right) .
\end{aligned}
$$

The off-diagonal entries of these matrices are fixed by the value $v_{w}$, so that the elements of the off-diagonal blocks $V_{d D^{\prime}}, V_{d \mathcal{D}^{\prime}}$ etc. in (3.10) are determined by the ratio of the electroweak scale to the masses of extra quark species. In the limit when the latter are very heavy they decouple and their mixings with light quarks become negligibly small. Thus, in this limit $3 \times 3$ block $V_{d d^{\prime}}$ becomes unitary. The same is true for analogous $V_{u u^{\prime}}$ block in up quark mixing. However, if the extra quarks are not that heavy and off-diagonal blocks are not negligible, then $V_{d d^{\prime}}$ and $V_{u u^{\prime}}$ blocks are no more unitary. 
The present experimental limits on the extra quark masses are $M_{U, D, Q}>(1 \div 1.5) \mathrm{TeV}$, depending on their type and decay modes [20]. Therefore, the ratios $\epsilon_{u}=v_{w} / M_{U}, \epsilon_{d}=$ $v_{w} / M_{D}$ and $\epsilon_{q}=v_{w} / M_{Q}$ can be considered as small parameters, $\epsilon_{u, d, q} \leq 0.1$ or so. By inspecting the matrices (3.11), one can estimate the elements of the off-diagonal blocks in $\mathcal{V}_{L, R}^{\text {down }}$ and $\mathcal{V}_{L, R}^{\text {up }}$ as

$$
\begin{aligned}
& \left|V_{d D^{\prime}}^{L}\right| \sim \epsilon_{d}, \quad\left|V_{u U^{\prime}}^{L}\right| \sim \epsilon_{u}, \quad\left|V_{d \mathcal{D}^{\prime}}^{L}\right|,\left|V_{u \mathcal{U}^{\prime}}^{L}\right| \sim \epsilon_{q}^{2} \\
& \left|V_{d \mathcal{D}^{\prime}}^{R}\right|,\left|V_{u \mathcal{U}^{\prime}}^{R}\right| \sim \epsilon_{q}, \quad\left|V_{d D^{\prime}}^{R}\right| \sim \epsilon_{d}^{2}, \quad\left|V_{u U^{\prime}}^{R}\right| \sim \epsilon_{u}^{2}
\end{aligned}
$$

modulo the Yukawa constants which are assumed to be $\leq 1$ for perturbativity. Therefore, the deviation from unitarity of the "left" matrices $V_{d d^{\prime}}^{L}$ and $V_{u u^{\prime}}^{L}$ blocks are $\sim \epsilon_{u, d}^{2} \leq 10^{-2}$. E.g. the first row unitarity of the matrix $\mathcal{V}_{\text {down }}^{L}$ implies $\left|V_{1 d}\right|^{2}+\left|V_{1 s}\right|^{2}+\left|V_{1 b}\right|^{2}=1-\left|V_{1 D^{\prime}}\right|^{2}-$ $\left|V_{1 \mathcal{D}^{\prime}}\right|^{2}=1-\delta_{d}$. Taking into account the above estimations, we see that the deviation can be as large as $\delta_{d} \sim \epsilon_{d}^{2} \sim 10^{-2}$. Let us recall that the CKM unitarity deficit $\delta_{\mathrm{CKM}}$ estimated in previous section is about $(1 \div 2) \times 10^{-3}$, see table 2 . Thus, for accounting for the above values of $\delta_{\mathrm{CKM}}$, one would need $\epsilon_{d}=0.03-0.05$. As for $\epsilon^{4} \sim 10^{-4}$ contributions, they are irrelevant and so order $\epsilon^{2}$ mixings as $V_{1 \mathcal{D}^{\prime}}$ etc. can be safely neglected.

Let us assume, for simplicity, that each of $U, D$ and $Q$ type species is present in one copy, i.e. $p=m=n=1$ (our discussion can be extended in a straightforward way for arbitrary number of extra species). In this case the off-diagonal blocks proportional to $v_{w}$ in $5 \times 5$ matrices (3.9) become columns as e.g. $h_{D}=\left(h_{1 D}, h_{2 D}, h_{3 D}\right)^{T}$ or rows as e.g. $h_{\mathcal{D}}=\left(h_{\mathcal{D} 1}, h_{\mathcal{D} 2}, h_{\mathcal{D} 3}\right)$. The Yukawa couplings $Y_{u, d}$ can be presented in the form $Y_{u}=$ $V_{L u} \widetilde{Y}_{u} V_{R u}^{\dagger}$ and $Y_{d}=V_{L d} \widetilde{Y}_{d} V_{R d}^{\dagger}$ where $\widetilde{Y}_{u, d}$ are diagonal $3 \times 3$ matrices, $\widetilde{Y}_{u}=\operatorname{diag}\left(y_{u}, y_{c}, y_{t}\right)$ and $\widetilde{Y}_{d}=\operatorname{diag}\left(y_{d}, y_{s}, y_{b}\right)$. Let us denote also $h_{U}=V_{L u} \widetilde{h}_{U}, h_{D}=V_{L d} \widetilde{h}_{D}, h_{\mathcal{U}}=\widetilde{h}_{\mathcal{U}} V_{R u}^{\dagger}$ and $h_{\mathcal{D}}=\widetilde{h}_{\mathcal{D}} V_{R d}^{\dagger}$.

Then for $5 \times 5$ unitary matrix of 'left' rotations we obtain, with the precision up to $\epsilon^{2}$ terms:

$$
\begin{aligned}
& \mathcal{V}_{\text {down }}^{L}=\left(\begin{array}{ccc}
V_{L d} & 0 & 0 \\
0 & 1 & 0 \\
0 & 0 & 1
\end{array}\right)\left(\begin{array}{ccc}
\sqrt{1-S_{D} S_{D}^{\dagger}} & S_{D} & 0 \\
-S_{D}^{\dagger} & \sqrt{1-S_{D}^{\dagger} S_{D}} & 0 \\
0 & 0 & 1
\end{array}\right)\left(\begin{array}{ccc}
\sqrt{1-S_{\mathcal{D}} S_{\mathcal{D}}^{\dagger}} & 0 & S_{\mathcal{D}} \\
0 & 1 & 0 \\
-S_{\mathcal{D}}^{\dagger} & 0 & \sqrt{1-S_{\mathcal{D}}^{\dagger} S_{\mathcal{D}}}
\end{array}\right) \\
& =\left(\begin{array}{ccc}
V_{L d}\left[1-\frac{1}{2} S_{D} S_{D}^{\dagger}-\frac{1}{2} S_{\mathcal{D}} S_{\mathcal{D}}^{\dagger}+\ldots\right] & V_{L d} S_{D} & V_{L d} S_{\mathcal{D}} \\
-S_{D}^{\dagger} & 1-\frac{1}{2} S_{D}^{\dagger} S_{D}+\ldots & O\left(\epsilon_{d} \epsilon_{q}^{2}\right) \\
-S_{\mathcal{D}}^{\dagger} & 0 & 1-\frac{1}{2} S_{\mathcal{D}}^{\dagger} S_{\mathcal{D}}+\ldots
\end{array}\right)
\end{aligned}
$$

where the column $S_{D}=\epsilon_{d} \widetilde{h}_{D}$ describes the light quark $(d, s, b)$ mixings with the extra isosinglet species $D$. As for their mixings with $\mathcal{D} \subset Q$ from extra isodoublet, $S_{\mathcal{D}}=\epsilon_{q}^{2} \widetilde{Y}_{d} \widetilde{h}_{\mathcal{D}}^{\dagger}$, it can be neglected since, apart of $\epsilon^{2}$ suppression, these are proportional to the small Yukawa constants $y_{d}, y_{s}, y_{b}$ in $\tilde{Y}_{d}$. Clearly, the unitarity conditions for rows and columns of this matrix is fulfilled with the precision up to $\sim \epsilon_{d}^{4}$ terms. The matrix $\mathcal{V}_{\text {up }}^{L}$ can be presented in an analogous form.

Let us discuss now charged current interactions. Considering that $q_{L}$ and $Q_{L}$ are $\mathrm{SU}(2)$ doublets while $U_{L}$ and $D_{L}$ are singlets, the LH charged current interacting with $W$ boson 
in terms of initial states and mass eigenstates reads:

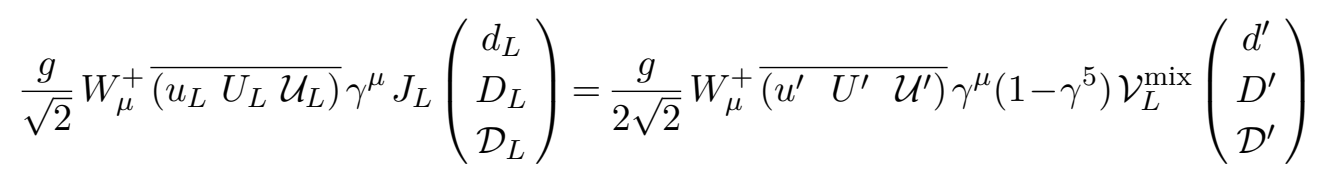

where $J_{L}=\operatorname{diag}(1,1,1,0,1)$ and $\mathcal{V}_{L}^{\text {mix }}=\left(\mathcal{V}_{L}^{\text {up }}\right)^{\dagger} J_{L} \mathcal{V}_{L}^{\text {down }}$, or explicitly

$$
\mathcal{V}_{L}^{\text {mix }}=\left(\begin{array}{ccc}
\left(1-\frac{1}{2} \Delta_{U}\right) \tilde{V}\left(1-\frac{1}{2} \Delta_{D}\right) & \left(1-\frac{1}{2} \Delta_{U}\right) \widetilde{V} S_{D} O\left(\epsilon^{2}\right) \\
S_{U}^{\dagger} \widetilde{V}\left(1-\frac{1}{2} \Delta_{D}\right) & S_{U}^{\dagger} \widetilde{V} S_{D} & O\left(\epsilon^{3}\right) \\
O\left(\epsilon^{2}\right) & O\left(\epsilon^{3}\right) & 1
\end{array}\right), \quad \widetilde{V}=V_{L u}^{\dagger} V_{L d}
$$

where $\Delta_{U}=S_{U} S_{U}^{\dagger}=\epsilon_{u}^{2} \widetilde{h}_{U} \widetilde{h}_{U}^{\dagger}$ and $\Delta_{D}=S_{D} S_{D}^{\dagger}=\epsilon_{d}^{2} \widetilde{h}_{D} \widetilde{h}_{D}^{\dagger}$ as far as $O\left(\epsilon_{q}^{4}\right)$ contributions $S_{\mathcal{U}} S_{\mathcal{U}}^{\dagger}$ and $S_{\mathcal{D}} S_{\mathcal{D}}^{\dagger}$ can be neglected.

We are interested in its $3 \times 3$ block which describes the transitions between the quark mass eigenstates $u^{\prime}=(u, c, t)$ and $d^{\prime}=(d, s, b)$ in charged current:

$$
V_{\mathrm{CKM}}=\left(1-\frac{1}{2} \Delta_{U}\right) \tilde{V}\left(1-\frac{1}{2} \Delta_{D}\right)=\left(\begin{array}{ccc}
V_{u d} & V_{u s} & V_{u b} \\
V_{c d} & V_{c s} & V_{c b} \\
V_{t d} & V_{t s} & V_{t b}
\end{array}\right)
$$

While $\widetilde{V}=V_{L u}^{\dagger} V_{L d}$ is unitary $3 \times 3$ matrix, the 'corrected' matrix $V_{\mathrm{CKM}}$ is not. In particular, deviation from the unitarity for its rows or columns read, up to order $\epsilon^{2}$ terms, respectively as ${ }^{10}$

$$
\begin{aligned}
& V_{\mathrm{CKM}} V_{\mathrm{CKM}}^{\dagger}=1-\epsilon_{u}^{2} \widetilde{h}_{U} \widetilde{h}_{U}^{\dagger}-\epsilon_{d}^{2} \widetilde{V} \widetilde{h}_{D} \widetilde{h}_{D}^{\dagger} \widetilde{V}^{\dagger}, \\
& V_{\mathrm{CKM}}^{\dagger} V_{\mathrm{CKM}}=1-\epsilon_{d}^{2} \widetilde{h}_{D} \widetilde{h}_{D}^{\dagger}-\epsilon_{u}^{2} \widetilde{V} \widetilde{h}_{U} \widetilde{h}_{U}^{\dagger} \widetilde{V}^{\dagger}
\end{aligned}
$$

In particular, the unitarity deficit for first row (2.1) we obtain $\delta_{C K M}=1-\left|V_{u d}\right|^{2}+\left|V_{u s}\right|^{2}+$ $\left|V_{u b}\right|^{2} \approx \epsilon_{u}^{2}\left|\widetilde{h}_{U 1}\right|^{2}+\epsilon_{d}^{2}\left|\widetilde{h}_{D 1}\right|^{2}$, which can fall in the range of $(1 \div 2) \times 10^{-3}$ provided that $\epsilon_{u}$ and/or $\epsilon_{d}$ are $\sim 0.1$ and the Yukawa constants $\widetilde{h}_{U 1}$ and $\widetilde{h}_{D 1}$ are large enough.

Let us discuss the $\mathrm{RH}$ sector. Considering that $Q_{R}$ is an $\mathrm{SU}(2)$ doublet while $u_{R}, d_{R}$, $U_{R}$ and $D_{R}$ are singlets, the $\mathrm{RH}$ charged current interacting with $W$ boson in terms of initial states and mass eigenstates reads:

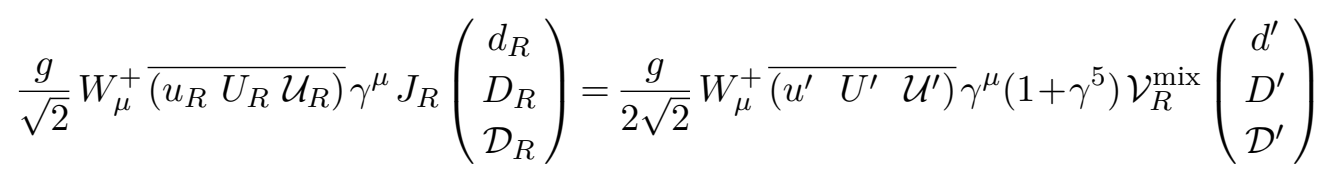

where $J_{R}=\operatorname{diag}(0,0,0,0,1)$ and $\mathcal{V}_{R}^{\text {mix }}=\left(\mathcal{V}_{R}^{\text {up }}\right)^{\dagger} J_{R} \mathcal{V}_{R}^{\text {down }}$. Thus, presenting matrices $\mathcal{V}_{R}^{\text {down }}$ which diagonalizes $\mathcal{M}_{\text {down }}^{\dagger} \mathcal{M}_{\text {down }}(3.11)$ in the form similar to $\mathcal{V}_{L}^{\text {down }}$ (3.13), and analogously doing for and $\mathcal{V}_{R}^{\text {up }}$, we get up to $O\left(\epsilon^{2}\right)$ terms:

$$
\mathcal{V}_{R}^{\text {mix }}=\left(\begin{array}{ccc}
S_{u} S_{d}^{\dagger} & 0 & -S_{u}\left(1-\frac{1}{2} S_{d}^{\dagger} S_{d}\right) \\
0 & 0 & 0 \\
-\left(1-\frac{1}{2} S_{u}^{\dagger} S_{u}\right) S_{d}^{\dagger} & 0 & 1-\frac{1}{2} S_{d}^{\dagger} S_{d}-\frac{1}{2} S_{u}^{\dagger} S_{u}
\end{array}\right)
$$

\footnotetext{
${ }^{10}$ Obviously, the 'large' mixing matrix $\mathcal{V}_{L}^{\text {mix }}$ is not unitary in itself because of the non-unitary factor $J_{L}$ 'sandwiched' between the unitary matrices $\mathcal{V}_{L}^{\text {up }}$ and $\mathcal{V}_{L}^{\text {down }}$.
} 
where $S_{d}=\epsilon_{q} \widetilde{h}_{\mathcal{D}}^{\dagger}$ and $S_{u}=\epsilon_{q} \widetilde{h}_{\mathcal{U}}^{\dagger}$. Thus, we see that the mixing with the weak isodoublet $Q$-type species induces $\mathrm{RH}$ charged current interactions between the quark mass eigenstates $u^{\prime}=(u, c, t)$ and $d^{\prime}=(d, s, b)$, given by the (non-unitary) $3 \times 3$ matrix:

$$
\Delta=\left(\begin{array}{ccc}
\Delta_{u d} & \Delta_{u s} & \Delta_{u b} \\
\Delta_{c d} & \Delta_{c s} & \Delta_{c b} \\
\Delta_{t d} & \Delta_{t s} & \Delta_{t b}
\end{array}\right)=S_{u} S_{d}^{\dagger}=\epsilon_{q}^{2} \widetilde{h}_{u}^{\dagger} \widetilde{h}_{d}=\epsilon_{q}^{2}\left(\begin{array}{ccc}
h_{u}^{*} h_{d} & h_{u}^{*} h_{s} & h_{u}^{*} h_{b} \\
h_{c}^{*} h_{d} & h_{c}^{*} h_{s} & h_{c}^{*} h_{b} \\
h_{t}^{*} h_{d} & h_{t}^{*} h_{s} & h_{t}^{*} h_{b}
\end{array}\right)
$$

where we denote the elements of row vectors $\widetilde{h}_{\mathcal{U}, \mathcal{D}}$ in the light quark mass basis as $\widetilde{h}_{\mathcal{U}}=\left(h_{u}, h_{c}, h_{t}\right)$ and $\widetilde{h}_{\mathcal{D}}=\left(h_{d}, h_{s}, h_{b}\right)$. Therefore, mixing with $Q$-type fermions violates pure $V-A$ character of the quark interactions with $W$ boson, and vector and axial couplings for each transition are not equal anymore but have a difference $O\left(\epsilon_{q}^{2}\right)$.

In fact, instead of purely $V-A$ couplings (1.1), now we have

$$
\frac{g}{2 \sqrt{2}} W_{\mu}^{+} \overline{\left(\begin{array}{lll}
u & c & t
\end{array}\right)}\left[\gamma^{\mu}\left(V_{\mathrm{CKM}}+\Delta\right)-\gamma^{\mu} \gamma^{5}\left(V_{\mathrm{CKM}}-\Delta\right)\right]\left(\begin{array}{l}
d \\
s \\
b
\end{array}\right)
$$

The presence of $\mathrm{RH}$ couplings has a direct implications for our problem. In particular, determination $\mathrm{C}$ from purely Fermi $0^{+}-0^{+}$transitions now fixes the vector coupling constant $G_{V}=G_{F}\left|V_{u d}+\Delta_{u d}\right|$, instead of $G_{F}\left|V_{u d}\right|$. Analogously, determination A from semileptonic decays $K \ell 3$, transforms in the determination of the vector coupling $\left|V_{u s}+\Delta_{u s}\right|$, instead of $\left|V_{u s}\right|$. On the other hand, since the leptonic decays $K \mu 2$ and $\pi \mu 2$ are contributed only by axial current, determination B instead of the ratio $\left|V_{u s} / V_{u d}\right|$ fixes the combination $\left|V_{u s}-\Delta_{u s}\right| /\left|V_{u d}-\Delta_{u d}\right|$. Therefore, instead of (2.10), (2.3) and (2.5), now we have:

$$
\begin{aligned}
\mathrm{C}: & & \left|V_{u d}+\Delta_{u d}\right| & =\left|V_{u d}\right|\left|1+\delta_{u d}\right|=0.97376(16) \\
\mathrm{A}: & & \left|V_{u s}+\Delta_{u s}\right| & =\left|V_{u s}\right|\left|1+\delta_{u s}\right|=0.22326(55) \\
& \mathrm{B}: & \left|\frac{V_{u s}-\Delta_{u s}}{V_{u d}-\Delta_{u d}}\right| & =\left|\frac{V_{u s}}{V_{u d}}\right|\left|\frac{1-\delta_{u s}}{1-\delta_{u d}}\right|=0.23130(49)
\end{aligned}
$$

where $\delta_{u d}=\Delta_{u d} / V_{u d}=\epsilon_{q}^{2} h_{u}^{*} h_{d} / V_{u d}$ and $\delta_{u s}=\Delta_{u s} / V_{u s}=\epsilon_{q}^{2} h_{u}^{*} h_{s} / V_{u s}$ are in general complex numbers. Hence, $Q$-type extra fermion can have interesting implications and potentially it can resolve all tensions between $\mathrm{A}, \mathrm{B}$ and $\mathrm{C}$ determinations. ${ }^{11}$

However, mixing with extra vector-like species (with non-standard isosipin content) affects the natural flavor conservation of the SM. Namely, by integrating out the heavy isosinglet states $D$ and $U$ one induces the following effective operators for the quark couplings with $Z$-boson

$$
\begin{aligned}
& \frac{g}{\cos \theta_{W}} \overline{q_{L i}} \gamma^{\mu}\left[\left(T_{3}-\mathrm{Q} \sin \theta_{W}^{2}\right) \delta_{i j}-T_{3} \frac{h_{D i} h_{D j}^{\dagger} \varphi^{\dagger} \varphi}{M_{D}^{2}}\right] q_{L j} Z_{\mu}, \\
& \frac{g}{\cos \theta_{W}} \overline{q_{L i}} \gamma^{\mu}\left[\left(T_{3}-\mathrm{Q} \sin \theta_{W}^{2}\right) \delta_{i j}-T_{3} \frac{h_{U i} h_{U j}^{\dagger} \tilde{\varphi}^{\dagger} \tilde{\varphi}}{M_{U}^{2}}\right] q_{L j} Z_{\mu},
\end{aligned}
$$

\footnotetext{
${ }^{11}$ In principle, one can obtain the $\mathrm{RH}$ weak currents also from left-right symmetric models. However, strong limits on $W_{R}$ mass imply that the mixing $W_{L}-W_{R}$ is too small to give the RH contributions needed to explain the anomalies.
} 

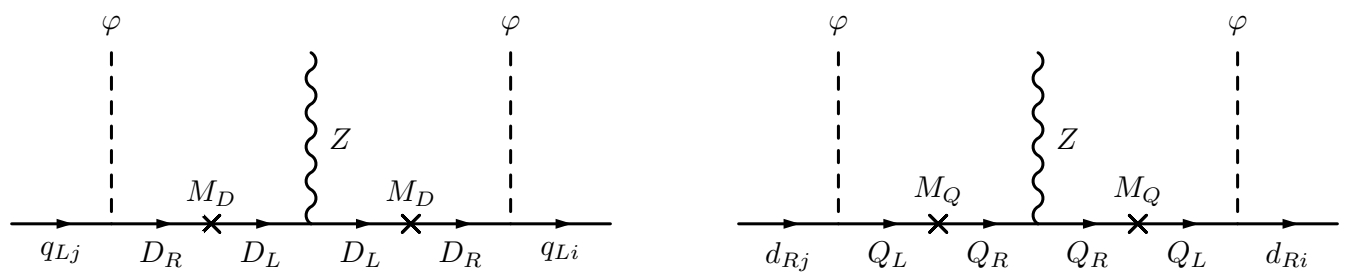

Figure 3. Anomolous flavour non-diagonal couplings of $Z$-boson with SM families, contributing to flavour changing processes at tree level.

as it is shown in figure 3 , where $T_{3}$ is the weak isospin and $\mathrm{Q}$ the electric charge, while mixing with the extra doublet $Q$ induces anomalous isospin violating couplings with the RH states

$$
\begin{aligned}
& \frac{g}{\cos \theta_{W}}\left[-\frac{1}{2} \frac{h_{d i}^{\dagger} h_{d j} \varphi^{\dagger} \varphi}{M_{Q}^{2}}+\frac{1}{3} \sin \theta_{W}^{2} \delta_{i j}\right] \overline{d_{R i}} \gamma^{\mu} d_{R j} Z_{\mu}, \\
& \frac{g}{\cos \theta_{W}}\left[\frac{1}{2} \frac{h_{u i}^{\dagger} h_{u j} \tilde{\varphi}^{\dagger} \tilde{\varphi}}{M_{Q}^{2}}-\frac{2}{3} \sin \theta_{W}^{2} \delta_{i j}\right] \overline{u_{R i}} \gamma^{\mu} u_{R j} Z_{\mu} .
\end{aligned}
$$

Thus, such anomalous couplings, flavor-non-diagonal between the mass eigenstates, after substitution of the VEV $\langle\phi\rangle=v_{w}$, contribute at tree level in the flavor changing phenomena as $K^{0}-\bar{K}^{0}$ mixing etc. inducing four-fermion effective operators

$$
-\frac{h_{D i} h_{D j}^{\dagger} h_{D k} h_{D m}^{\dagger} v_{w}^{2}}{4 M_{D}^{4}}\left(\overline{d_{L i}} \gamma^{\mu} d_{L j}\right)\left(\overline{d_{L k}} \gamma_{\mu} d_{L m}\right), \quad-\frac{h_{U i} h_{U j}^{\dagger} h_{U k} h_{U m}^{\dagger} v_{w}^{2}}{4 M_{U}^{4}} \overline{u_{L i}} \gamma^{\mu} u_{L j} \overline{u_{L k}} \gamma^{\mu} u_{L m}
$$

and analogously for RH states. These operators parametrically are order $G_{F}\left(v_{w} / M\right)^{4} \sim$ $v_{w}^{2} / M^{4}$. On the other hand, box diagrams shown in the upper-left part of figure 4 (and analogously for up-type quarks) induce operators which parametrically are order $1 / M^{2}$ :

$$
\frac{h_{D i} h_{D j}^{\dagger} h_{D k} h_{D m}^{\dagger}}{128 \pi^{2} M_{D}^{2}}\left(\overline{d_{L i}} \gamma^{\mu} d_{L j}\right)\left(\overline{d_{L k}} \gamma_{\mu} d_{L m}\right), \quad \frac{h_{U i} h_{U j}^{\dagger} h_{U k} h_{U m}^{\dagger}}{128 \pi^{2} M_{U}^{2}} \overline{u_{L i}} \gamma^{\mu} u_{L j} \overline{u_{L k}} \gamma^{\mu} u_{L m}
$$

but are suppressed by a loop factor. Regarding the RH sector, as in the upper-right part of figure 4 we have:

$$
\frac{h_{d i}^{\dagger} h_{d j} h_{d k}^{\dagger} h_{d m}}{64 \pi^{2} M_{D}^{2}}\left(\overline{d_{R i}} \gamma^{\mu} d_{R j}\right)\left(\overline{d_{R k}} \gamma_{\mu} d_{R m}\right), \quad \frac{h_{u i}^{\dagger} h_{u j} h_{u k}^{\dagger} h_{u m}}{64 \pi^{2} M_{U}^{2}} \overline{u_{R i}} \gamma^{\mu} u_{R j} \overline{u_{R k}} \gamma^{\mu} u_{R m}
$$

In the presence of all type of fermions, also left-right operators can be induced, as shown in figure 4 (bottom).

In addition, extra vector-like leptons induce analogous contributions in lepton sector. However, in the following, for brevity, we shall concentrate only on quark sector, and study one by one implications of $D$-type, $U$-type and $Q$-type extra fermions. 

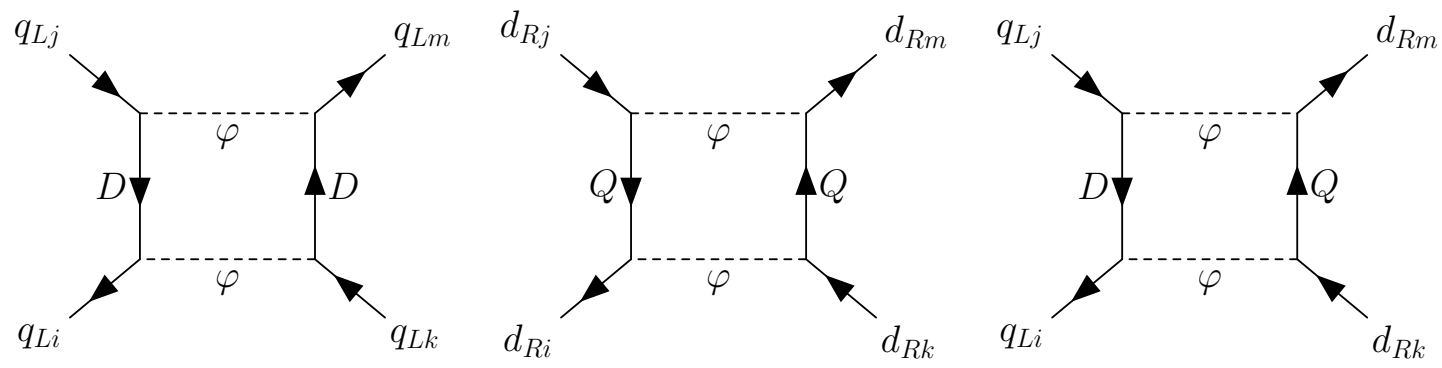

Figure 4. Box diagrams induced by the presence of vector-like quarks.

Namely, in each of these cases we put limits that emerge from flavour changing phenomena $\left(K^{0}-\bar{K}^{0}, D^{0}-\bar{D}^{0}, B^{0}-\bar{B}^{0}\right.$, flavour changing meson decays) and flavour conserving observables ( $Z$-boson physics, low energy observables). Our aim is to understand if parameter space remains for vector-like fermions to contribute at the level needed for fixing the Cabibbo anomalies. Our strategy is to consider each process separately and see the parameter space left available, in order to make clear how much constraining each process is. Then, it can be inferred for each process the needed level of progress in accuracy and sensitivity which can possibly show this new physics. Moreover, since our theoretical knowledge is different depending on the process, in this way we can separate limits obtained from golden modes, which do not suffer from large long distance contributions and are theoretically clean. Since we made rather conservative choices for the bounds, we present results so that limits can be rescaled according to increasing accuracy or different assumptions. For down-type weak singlets these limits were first discussed in ref. [80]. However in that work contributions of box diagrams involving heavy states were not discussed, whereas, as we will show, these diagrams become dominant if heavy vector-like quarks have masses larger than $\sim 3 \mathrm{TeV}$. The whole of these operators was considered in ref. [81].

\section{Extra down-type isosinglet}

Let us examine in details the implications of the addition of a down-type vector-like weak isosinglet (D-type) couple of quarks $D_{L}=d_{L 4}$ and $D_{R}=d_{R 4}$ involved in the mixing with the SM three families $q_{L i}=\left(u_{L i}, d_{L i}\right)^{T}, u_{R i}$ and $d_{R i}, i=1,2,3$. New Yukawa terms and Dirac mass terms should be added to the Lagrangian density besides the standard Yukawa terms: $h_{d j}^{\prime} \varphi \overline{q_{L j}} d_{R i}^{\prime}+m_{i} \overline{d_{L 4}} d_{R i}^{\prime}+$ h.c.. Since the four species of right-handed singlets $d_{R i}^{\prime}$ have identical quantum numbers, a unitary transformation can be applied on the four components $d_{R i}^{\prime}$ so that $m_{j}=0$ for $j=1,2,3$ and $d_{R 4}$ is identified with the combination making the Dirac mass term with the left handed singlet $d_{L 4}$. Thus the Yukawa Lagrangian of this system can be written as:

$$
y_{i j}^{u} \tilde{\varphi} \overline{q_{L i}} u_{R j}+y_{i j}^{d} \varphi \overline{q_{L i}} d_{R j}+h_{d j} \varphi \overline{q_{L j}} d_{R 4}+M_{4 d} \overline{d_{L 4}} d_{R 4}+\text { h.c. }
$$


where, without losing generality, the mass term $M_{4 d}$ can be taken real and positive. Then the down-type quarks mass matrix looks like:

$$
\begin{aligned}
& \overline{d_{L i}} \mathbf{m}_{i j}^{(d)} d_{R j}+\text { h.c. }=
\end{aligned}
$$

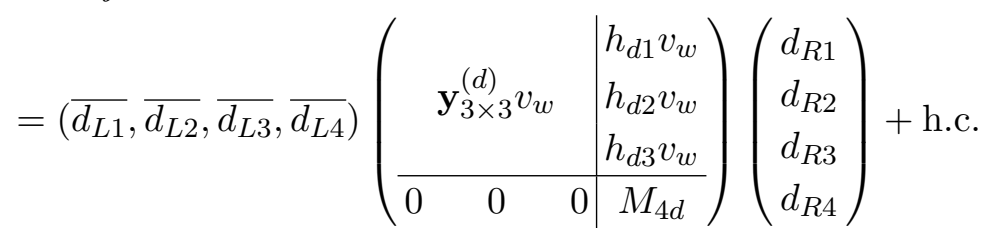

where $v_{w}=174 \mathrm{GeV}$ is the SM Higgs vacuum expectation value (VEV) (for a convenience, we use this normalization of the Higgs VEV instead of the "standard" normalization $\langle\phi\rangle=v / \sqrt{2}$, i.e. $\left.v=\sqrt{2} v_{\mathrm{w}}\right)$ and $\mathbf{y}_{3 \times 3}^{(d)}$ is the $3 \times 3$ matrix of Yukawa couplings. The mass matrix $\mathbf{m}^{(d)}$ can be diagonalized with positive eigenvalues by a biunitary transformation:

$$
V_{L}^{(d) \dagger} \mathbf{m}^{(d)} V_{R}^{(d)}=\mathbf{m}_{\text {diag }}^{(d)}=\operatorname{diag}\left(y_{d} v_{w}, y_{s} v_{w}, y_{b} v_{w}, M_{b^{\prime}}\right)
$$

where $V_{L, R}^{(d)}$ are two unitary $4 \times 4$ matrices. $\mathbf{m}_{\text {diag }}^{(d)}$ is the diagonal matrix of mass eigenvalues $m_{d, s, b}=y_{d, s, b} v_{w}$ and $M_{b^{\prime}} \approx M_{4 d}$. Weak eigenstates in terms of mass eigenstates are:

$$
\left(\begin{array}{c}
d_{L 1} \\
d_{L 2} \\
d_{L 3} \\
d_{L 4}
\end{array}\right)=V_{L}^{(d)}\left(\begin{array}{c}
d_{L} \\
s_{L} \\
b_{L} \\
b_{L}^{\prime}
\end{array}\right), \quad V_{L}^{(d)}=\left(\begin{array}{cccc}
V_{L 1 d} & V_{L 1 s} & V_{L 1 b} & V_{L 1 b^{\prime}} \\
V_{L 2 d} & V_{L 2 s} & V_{L 2 b} & V_{L 2 b^{\prime}} \\
V_{L 3 d} & V_{L 3 s} & V_{L 3 b} & V_{L 3 b^{\prime}} \\
V_{L 4 d} & V_{L 4 s} & V_{L 4 b} & V_{L 4 b^{\prime}}
\end{array}\right)
$$

As for up-type quarks, the up-quark Yukawa matrix can be taken diagonal, $\mathbf{y}^{u}=\operatorname{diag}\left(y_{u}\right.$, $\left.y_{c}, y_{t}\right)$, so that also the mass matrix $\mathbf{m}_{u}=\operatorname{diag}\left(m_{u}, m_{c}, m_{t}\right)=\operatorname{diag}\left(y_{u}, y_{c}, y_{t}\right) \times v_{w}$ is diagonal, and $u_{1}, u_{2}, u_{3}$ correspond to the mass eigenstates $u, c, t$. Since only the three down-type quarks $d_{L 1}, d_{L 2}, d_{L 3}$ couple with $W$-bosons, the Lagrangian for the charged weak interactions expressed in terms of mass eigenstates become:

$$
\begin{aligned}
\mathcal{L}_{\mathrm{cc}} & =\frac{g}{\sqrt{2}}\left(\overline{u_{L 1}} \overline{u_{L 2}} \overline{u_{L 3}}\right) \gamma^{\mu}\left(\begin{array}{c}
d_{L 1} \\
d_{L 2} \\
d_{L 3}
\end{array}\right) W_{\mu}^{+}+\text {h.c. }= \\
& =\frac{g}{\sqrt{2}}\left(\overline{u_{L}} \overline{c_{L}} \overline{t_{L}}\right) \gamma^{\mu} \tilde{V}_{\mathrm{CKM}}\left(\begin{array}{c}
d_{L} \\
s_{L} \\
b_{L} \\
b_{L}^{\prime}
\end{array}\right) W_{\mu}^{+}+\text {h.c. }
\end{aligned}
$$

where

$$
\tilde{V}_{\mathrm{CKM}}=\tilde{V}_{L}^{(d)}=\left(\begin{array}{cccc}
V_{L 1 d} & V_{L 1 s} & V_{L 1 b} & V_{L 1 b^{\prime}} \\
V_{L 2 d} & V_{L 2 s} & V_{L 2 b} & V_{L 2 b^{\prime}} \\
V_{L 3 d} & V_{L 3 s} & V_{L 3 b} & V_{L 3 b^{\prime}}
\end{array}\right)=\left(V_{\mathrm{CKM}} \mid \begin{array}{c}
V_{u b^{\prime}} \\
V_{c b^{\prime}} \\
V_{t b^{\prime}}
\end{array}\right)=\left(\begin{array}{cccc}
V_{u d} & V_{u s} & V_{u b} & V_{u b^{\prime}} \\
V_{c d} & V_{c s} & V_{c b} & V_{c b^{\prime}} \\
V_{t d} & V_{t s} & V_{t b} & V_{t b^{\prime}}
\end{array}\right)
$$

is the $3 \times 4$ submatrix of $V_{L}^{(d)}$ in eq. (4.4), obtained by cutting the last row. Obviously, $\tilde{V}_{\mathrm{CKM}}$ is not unitary anymore. More precisely, although the unitarity condition is violated 


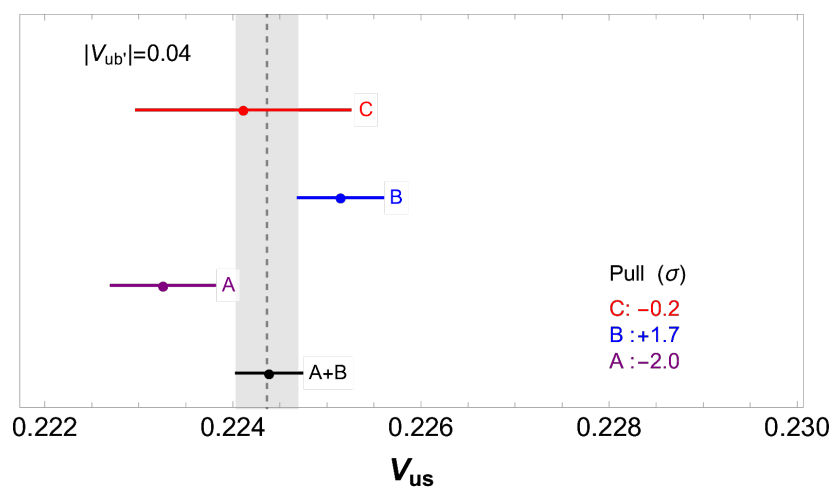

Figure 5. Determinations of $\left|V_{u s}\right|$ obtained using eq. (4.7) with $\left|V_{u b^{\prime}}\right|=0.043$, with the dataset in eqs. (2.3), (2.5), (2.14), to be compared with figure 2 .

\begin{tabular}{|lcc|}
\hline Determination & $\left|V_{u s}\right|$ value & $\left|V_{u b^{\prime}}\right|=0.043$ \\
\hline A & $0.22326(55)$ & $0.22326(55)$ \\
B & $0.22535(45)$ & $0.22515(45)$ \\
C & $0.2282(11)$ & $0.2241(11)$ \\
A+B & $0.22451(35)$ & $0.22439(35)$ \\
A+B + C & $0.22482(33)$ & $0.22436(33)$ \\
\hline
\end{tabular}

Table 3. Values of $V_{u s}$ obtained from the dataset in eqs. (2.3), (2.5), (2.14). In the first column the SM unitarity of CKM matrix is used, while in the second column the extended unitarity (4.7) is used with $\left|V_{u b^{\prime}}\right|=0.043$.

for columns, it still holds for rows: $\tilde{V}_{L}^{(d)} \tilde{V}_{L}^{(d) \dagger}=1_{3 \times 3}$, with $1_{3 \times 3}$ being the $3 \times 3$ identity matrix. In particular, the first row unitarity condition of CKM matrix is modified to

$$
\left|V_{u d}\right|^{2}+\left|V_{u s}\right|^{2}+\left|V_{u b}\right|^{2}+\left|V_{u b^{\prime}}\right|^{2}=1
$$

which is the extended unitarity condition for the first row. The elements of the fourth column of $\tilde{V}_{\mathrm{CKM}}$ determine the strength of the violation of SM CKM unitarity.

The dataset A, B, C from eqs. (2.3), (2.5), (2.10) can be fitted in this scenario by using the extended unitarity (4.7). The best fit point $\left(\chi_{\text {dof }}^{2}=7.1\right)$ is obtained in $\left|V_{u s}\right|=$ $0.22436(36)$, with:

$$
\left|V_{u b^{\prime}}\right|^{2}=1.83(55) \times 10^{-3}, \quad\left|V_{u b^{\prime}}\right|=0.043(7)
$$

At $95 \%$ C.L. the needed additional mixing is $\left|V_{u b^{\prime}}\right|=0.043_{-0.015}^{+0.011}$. In figure 5 it is shown how the present situation would look like by choosing $\left|V_{u b^{\prime}}\right|=0.043$. The data are listed in table 3. Determinations $\mathrm{B}$ and $\mathrm{C}$ are shifted with respect to the ones in figure 2, while A remains unchanged. Determination $\mathrm{C}$, which is obtained from $V_{u d}$ from beta decays, becomes aligned with the average of the determinations of $V_{u s}$ obtained from kaon decays, because of the extended unitarity relation (4.7). The $\chi^{2}$ is rather large $\chi^{2}=7.1$, because of the remaining tension between the determinations of $V_{u s}$ from kaon physics (A and B). 
However, the mixing $V_{L}^{(d)}$ induces non-standard couplings of $Z$-boson with the LH down quarks, since the normal families $d_{L i}, i=1,2,3$ reside in doublets while $d_{L 4}$ is a weak singlet (the mixing $V_{R}^{(d)}$ of the $\mathrm{RH}$ quarks does not give the same effect since all $\mathrm{RH}$ states $d_{R i}$ are in identical representations of the SM). In fact, $Z$-boson couples to a fermion species $f(\mathrm{LH}$ or $\mathrm{RH})$ as $Z_{\mu} \bar{f}\left(T_{3}+Q \sin ^{2} \theta_{W}\right) f$, where $T_{3}$ is the weak isospin projection and $Q$ the electric charge. Therefore, $Q$-dependent couplings remain diagonal between the mass eigenstates $d, s, b, b^{\prime}$, while the $T_{3}$ dependent part gets non-diagonal couplings. The weak neutral current Lagrangian for down quarks reads:

$$
\begin{aligned}
\mathcal{L}_{\mathrm{nc}} & =\frac{g}{\cos \theta_{W}}\left[-\frac{1}{2}\left(\overline{d_{L 1}} \overline{d_{L 2}} \overline{d_{L 3}}\right) \gamma^{\mu}\left(\begin{array}{c}
d_{L 1} \\
d_{L 2} \\
d_{L 3}
\end{array}\right)+\frac{1}{3} \sin ^{2} \theta_{W}\left(\overline{\mathbf{d}_{L}} \gamma^{\mu} \mathbf{d}_{L}+\overline{\mathbf{d}_{R}} \gamma^{\mu} \mathbf{d}_{R}\right)\right] Z_{\mu}= \\
& =\frac{g}{\cos \theta_{W}}\left[-\frac{1}{2}\left(\overline{d_{L}} \overline{s_{L}} \overline{b_{L}} \overline{b_{L}^{\prime}}\right) \gamma^{\mu} V_{\mathrm{nc}}^{(d)}\left(\begin{array}{c}
d_{L} \\
s_{L} \\
b_{L} \\
b_{L}^{\prime}
\end{array}\right)+\frac{1}{3} \sin ^{2} \theta_{W}\left(\overline{\mathbf{d}_{L}} \gamma^{\mu} \mathbf{d}_{L}+\overline{\mathbf{d}_{R}} \gamma^{\mu} \mathbf{d}_{R}\right)\right] Z_{\mu} \\
V_{\mathrm{nc}}^{(d)} & =V_{L}^{(d) \dagger} \operatorname{diag}(1,1,1,0) V_{L}^{(d)}=\tilde{V}_{L}^{(d) \dagger} \tilde{V}_{L}^{(d)}
\end{aligned}
$$

where $\mathbf{d}$ is the column vector of the four down-type quarks $d, s, b, b^{\prime}$. As comes out from eq. (4.9), the non-unitarity of $\tilde{V}_{L}^{(d)}$ is at the origin of non-diagonal couplings with $Z$ boson, which means flavor changing neutral currents (FCNC) at tree level. Explicitly, the weak isospin dependent part of the $Z$ coupling is given by:

$$
\begin{aligned}
& V_{\mathrm{nc}}^{(d)}=V_{L}^{(d) \dagger} \operatorname{diag}(1,1,1,0) V_{L}^{(d)}= \\
& =\left(\begin{array}{llll}
1 & 0 & 0 & 0 \\
0 & 1 & 0 & 0 \\
0 & 0 & 1 & 0 \\
0 & 0 & 0 & 0
\end{array}\right)+\left(\begin{array}{cccc}
-\left|V_{L 4 d}\right|^{2} & -V_{L 4 d}^{*} V_{L 4 s} & -V_{L 4 d}^{*} V_{L 4 b} & -V_{L 4 d}^{*} V_{L 4 b^{\prime}} \\
-V_{L 4 s}^{*} V_{L 4 d} & -\left|V_{L 4 s}\right|^{2} & -V_{L 4 s}^{*} V_{L 4 b} & -V_{L 4 s}^{*} V_{L 4 b^{\prime}} \\
-V_{L 4 b}^{*} V_{L 4 d} & -V_{L 4 b}^{*} V_{L 4 s} & -\left|V_{L 4 b}\right|^{2} & -V_{L 4 b}^{*} V_{L 4 b^{\prime}} \\
-V_{L 4 b^{\prime}}^{*} V_{L 4 d} & -V_{L 4 b^{\prime}}^{*} V_{L 4 s} & -V_{L 4 b^{\prime}}^{*} V_{L 4 b} & \left|V_{L 1 b^{\prime}}\right|^{2}+\left|V_{L 2 b^{\prime}}\right|^{2}+\left|V_{L 3 b^{\prime}}\right|^{2}
\end{array}\right)
\end{aligned}
$$

$V_{L}^{(d)}$ can be parameterized by 6 angles and 10 phases. However, four phases can be eliminated by phase transformations of $d, s, b, b^{\prime}$ states, so that $V_{L}^{(d)}$ can be presented as:

$$
\begin{aligned}
& V_{L}^{(d)}=\left(\begin{array}{llll}
V_{L 1 d} & V_{L 1 s} & V_{L 1 b} & V_{L 1 b^{\prime}} \\
V_{L 2 d} & V_{L 2 s} & V_{L 2 b} & V_{L 2 b^{\prime}} \\
V_{L 3 d} & V_{L 3 s} & V_{L 3 b} & V_{L 3 b^{\prime}} \\
V_{L 4 d} & V_{L 4 s} & V_{L 4 b} & V_{L 4 b^{\prime}}
\end{array}\right) \simeq V_{3 L}^{(d)} L^{(d)}=
\end{aligned}
$$

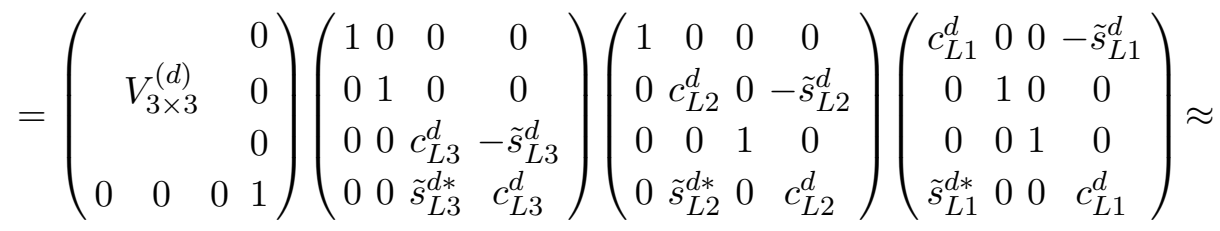

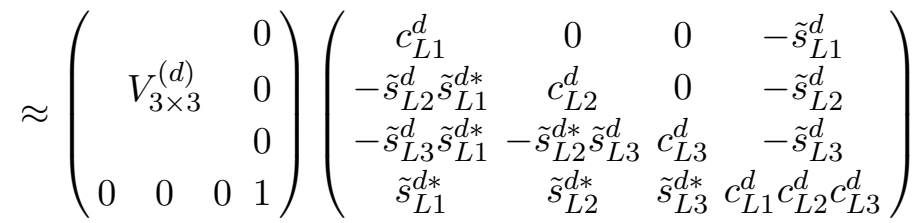


$c_{L i}^{d}$ are cosines and $\tilde{s}_{L i}^{d}$ are complex sines of angles in the 14,24, 34 family planes parameterizing the mixing of the first three families with the vector-like quark:

$$
\tilde{s}_{L i}^{d}=\sin \theta_{L i 4}^{d} e^{i \delta_{L i}^{d}}=s_{L i}^{d} e^{i \delta_{L i}^{d}}, \quad c_{L i}^{d}=\cos \theta_{L i 4}^{d}, \quad \delta_{L i j}^{d}=\delta_{L i}^{d}-\delta_{L j}^{d}
$$

and corresponding to the elements of the last row:

$$
V_{L 4 d} \approx \tilde{s}_{L 1}^{d *}, \quad V_{L 4 s} \approx \tilde{s}_{L 2}^{d *}, \quad V_{L 4 b}=\tilde{s}_{L 3}^{d *}
$$

Since it is the relative phase of the elements which will come into play, we also defined the relative phase of the elements in eq. (4.12). $V_{3 L}^{(d)}$ contains 3 angles and 3 phases; it diagonalizes the $3 \times 3$ Yukawa matrix $\mathbf{y}_{3 \times 3}^{(d)}$ in eq. (4.2). Since the elements in $V_{3 L}^{(d)}$ are small, $h_{d i}=V_{L i j}^{(d)} \tilde{h}_{d j} \approx \tilde{h}_{d i}$. Equation (4.11) is true at order $O\left(\left|h_{d i}\right|\left(y_{i}\right)^{2} \frac{v_{w}^{3}}{M_{b^{\prime}}^{3}}+\left|h_{d j}\right|^{2}\left|h_{d i}\right| \frac{v_{w}^{3}}{M_{b^{\prime}}^{3}}\right)$, $i, j=1,2,3, y_{i}=y_{d, s, b}$, with:

$$
\frac{1}{2} \tan \left(2 \theta_{L i 4}^{d}\right) \approx \frac{\left|h_{d i}\right| v_{w}}{M_{b^{\prime}}}, \quad \delta_{L i}^{d}=\arg \left(h_{d i}\right)-\pi
$$

that is:

$$
\tilde{s}_{L i}^{d} \approx-\frac{h_{d i} v_{w}}{M_{b^{\prime}}}
$$

Because of small mixing angles, $V_{3 \times 3}^{(d)}$ is practically equal to the $3 \times 3$ submatrix of $V_{L}^{(d)}$ in (4.4). In fact, for example in the chosen parameterization (4.11), the main corrections regard the elements: $V_{L 3 d} \simeq V_{3 \times 33 d}-\tilde{s}_{L 1}^{d} \tilde{s}_{L 3}^{d}, V_{L 2 d} \simeq V_{3 \times 32 d}-\tilde{s}_{L 1}^{d} \tilde{s}_{L 2}^{d}, V_{L 3 s} \simeq V_{3 \times 33 s}-$ $\tilde{s}_{L 2}^{d} \tilde{s}_{L 3}^{d}, V_{L 1 d} \simeq V_{3 \times 31 d} c_{L 1}^{d}$. However, it will be shown that, in order to have $s_{L 1}^{d} \approx\left|V_{L 4 d}\right| \approx$ $\left|V_{u b^{\prime}}\right| \approx 0.03$, it should be that at most $s_{L 3}^{d}=\left|V_{L 4 b}\right|<7.4 \times 10^{-3}$ and $s_{L 2}^{d} \approx\left|V_{L 4 s}\right|<$ $5.0 \times 10^{-4}$. Then:

$$
\left[V_{L}^{(d)}\right]_{i \beta} \simeq\left[V_{3 \times 3}^{(d)}\right]_{i \beta}
$$

As regards charged currents, $\tilde{V}_{\mathrm{CKM}}$ in (4.6) can be described by 6 moduli and 9 phases, 6 of which can be absorbed into the quark fields. For the submatrix $V_{\mathrm{CKM}}$ in (4.6), it holds that:

$$
\left[V_{\mathrm{CKM}}\right]_{\alpha \beta}=\sum_{i=1}^{3} V_{L i \alpha}^{(u) *} V_{L i \beta}^{(d)} \simeq \sum_{i=1}^{3} V_{L i \alpha}^{(u) *} V_{3 \times 3 i \beta}^{(d)}
$$

Then, after rephasing the quark fields, $V_{\mathrm{CKM}}$ can be in the usual parameterization with 3 angles and one phase. Also another phase can be absorbed, so we can always choose $\delta_{L 1}^{d}=0$ without loss of generality. From (4.17), for the elements of the fourth column of $\tilde{V}_{\text {CKM }}$ in (4.6) it holds that:

$$
\begin{aligned}
& V_{u b^{\prime}} \approx-V_{L 4 d}^{*} V_{u d}-V_{L 4 s}^{*} V_{u s}-V_{L 4 b}^{*} V_{u b} \approx-V_{L 4 d}^{*} \\
& V_{c b^{\prime}} \approx-V_{L 4 d}^{*} V_{c d}-V_{L 4 s}^{*} V_{c s}-V_{L 4 b}^{*} V_{c b} \\
& V_{t b^{\prime}} \approx-V_{L 4 d}^{*} V_{t d}-V_{L 4 s}^{*} V_{t s}-V_{L 4 b}^{*} V_{t b} \simeq-V_{L 4 b}^{*}
\end{aligned}
$$

where the last approximations come from the mentioned constraints on the mixings. 
It should be noted that also the couplings of quarks with the real Higgs are not diagonal if the vector-like species are added. In fact, the matrix of Yukawa couplings and the mass matrix (4.2) are not proportional anymore, and then they are not diagonalized by the same transformation. In particular, left-handed light quarks are coupled with $b_{R}^{\prime}$ with coupling constants which can be in principle of order $O(1)$. In fact, the Higgs couplings with quarks are:

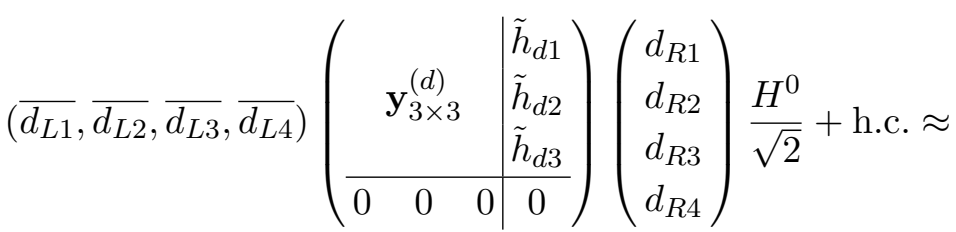

$$
\begin{aligned}
& \approx\left(\overline{d_{L}}, \overline{s_{L}}, \overline{b_{L}}, \overline{b_{L}^{\prime}}\right) L^{(d) \dagger}\left(\begin{array}{cccc}
y_{d} & 0 & 0 & h_{d 1} \\
0 & y_{s} & 0 & h_{d 2} \\
0 & 0 & y_{b} & h_{d 3} \\
0 & 0 & 0 & 0
\end{array}\right) R^{(d)}\left(\begin{array}{c}
d_{R} \\
s_{R} \\
b_{R} \\
b_{R}^{\prime}
\end{array}\right) \frac{H^{0}}{\sqrt{2}}+\text { h.c. } \approx \\
& \approx\left(\overline{d_{L}}, \overline{s_{L}}, \overline{b_{L}}, \overline{b_{L}^{\prime}}\right)\left(\begin{array}{cccc}
c_{L 1}^{d} y_{d} & \tilde{s}_{R 2}^{d *} h_{d 1} & \tilde{s}_{R 3}^{d *} h_{d 1} & h_{d 1} \\
\tilde{s}_{R 1}^{d *} h_{d 2} & y_{s} & \tilde{s}_{R 3}^{d *} h_{d 2} & h_{d 2} \\
\tilde{s}_{R 1}^{d *} h_{d 3} & \tilde{s}_{R 2}^{d *} h_{d 3} & y_{b} & h_{d 3} \\
-\tilde{s}_{L 1}^{d *} y_{d} & -\tilde{s}_{L 2}^{d *} y_{s} & -\tilde{s}_{L 3}^{d *} y_{b} & \sum_{i=1}^{3}\left(-\tilde{s}_{L i}^{d *} h_{d i}\right)
\end{array}\right)\left(\begin{array}{c}
d_{R} \\
s_{R} \\
b_{R} \\
b_{R}^{\prime}
\end{array}\right) \frac{H^{0}}{\sqrt{2}}+\text { h.c. }
\end{aligned}
$$

where $L^{(d)}$ is defined in (4.11) and $R^{(d)}$ is analogously defined. Then in principle FC couplings between light quarks emerge at tree level. However the mixing angles of the SM right-handed quarks with $b_{R}^{\prime}$ are much smaller than angles in the left-handed sector, $s_{R i}^{d} \approx \frac{y_{i}\left|h_{d i}\right| v_{w}^{2}}{M_{b^{\prime}}^{2}}$, where $s_{R i}^{d}$ is defined in the same way as $s_{L i}^{d}$ in eqs. (4.11), (4.12).

It should also be noticed that, because of the large mixing with the first family, the extra quark $b^{\prime}$ would mainly decay into $u$ or $d$ quark via the couplings with $W, Z, H$. The CMS experiment put lower limits on the mass of vector-like quarks coupling to light quarks, which in our scenario imply $M_{b^{\prime}} \gtrsim 700 \mathrm{GeV}$ [82]. It should be noticed that, with this constraint, $\left|V_{u b^{\prime}}\right| \simeq 0.03$ can be obtained if $\left|h_{d 1}\right| \gtrsim 0.1$, much larger than the Yukawa constant of the bottom quark $y_{b}$. In turn, by taking $\left|V_{u b^{\prime}}\right|>0.03$ in $M_{b^{\prime}}=\left|h_{d 1}\right| v_{w} /\left|V_{u b^{\prime}}\right|$, and assuming (for the perturbativity) $\left|h_{d 1}\right| \lesssim 1$, there is an upper limit on the extra quark mass, $M_{b^{\prime}} \lesssim 6 \mathrm{TeV}$.

In the following sections experimental limits from FCNC and electroweak observables are examined. The results are summarized in section 4.5, in table 6 and figures 11, 12 .

In table 4 well determined basic quantities and SM parameters employed in the computations are collected. Values are taken from Particle Data Group (PDG) [20].

\subsection{Limits from rare kaon decays}

\subsection{1 $\quad K^{+} \rightarrow \pi^{+} \nu \bar{\nu}$}

The decay $K^{+} \rightarrow \pi^{+} \nu \bar{\nu}$ is one of the golden modes for testing the SM, since long-distance contributions are negligibly small. The effective Lagrangian comes from a combination of 


\begin{tabular}{|lrlr|}
\hline Quantity & Value & Quantity & Value \\
\hline$\left|V_{c d}\right|$ & $0.221(4)$ & $M_{t}[\mathrm{GeV}]$ & $172.76(30)$ \\
$\left|V_{c b}\right|$ & $0.0410(14)$ & $m_{H}[\mathrm{GeV}]$ & $125.10(14)$ \\
$\left|V_{t s}\right|$ & $0.0388(11)$ & $m_{c}\left(m_{c}\right)[\mathrm{GeV}]$ & $1.27(2)$ \\
$\left|V_{t d}\right|$ & $0.0080(3)$ & $m_{b}\left(m_{b}\right)[\mathrm{GeV}]$ & $4.18(3)$ \\
$\left|V_{u b}\right|$ & $0.00382(24)$ & $m_{K^{0}}[\mathrm{MeV}]$ & $497.611(13)$ \\
$\left|V_{c s}\right|$ & $0.987(11)$ & $\tau_{K_{L}}[\mathrm{~s}]$ & $5.116(21) \cdot 10^{-8}$ \\
$\lambda$ & $0.22653(48)$ & $\tau_{K_{S}}[\mathrm{~s}]$ & $8.954(4) \cdot 10^{-11}$ \\
$\bar{\rho}$ & $0.123(32)$ & $M_{K^{+}}[\mathrm{MeV}]$ & $493.677(16)$ \\
$A$ & $0.799(28)$ & $\tau_{K^{+}}[\mathrm{s}]$ & $1.2380(20) \cdot 10^{-8}$ \\
$\bar{\eta}$ & $0.382(29)$ & $\tau_{D^{+}}[\mathrm{s}]$ & $1.040(7) \cdot 10^{-12}$ \\
$\alpha_{s}\left(M_{Z}\right)$ & $0.1185(16)$ & $M_{D^{+}}[\mathrm{MeV}]$ & $1869.65(5)$ \\
$\alpha\left(M_{Z}\right)^{-1}$ & $127.952(9)$ & $\tau_{D^{0}}[\mathrm{~s}]$ & $4.101(15) \cdot 10^{-13}$ \\
$\sin ^{2} \theta_{W}\left(M_{Z}\right)$ & $0.23121(4)$ & $m_{D^{0}}[\mathrm{MeV}]$ & $1864.83(5)$ \\
$G_{F}\left[\mathrm{GeV}{ }^{-2}\right]$ & $1.1663787(6) \cdot 10^{-5}$ & $\tau_{B_{d}^{0}}[\mathrm{~s}]$ & $1.519(4) \cdot 10^{-12}$ \\
$m_{W}[\mathrm{GeV}]$ & $80.379(12)$ & $M_{B_{d}^{0}}[\mathrm{MeV}]$ & $5279.65(12)$ \\
$m_{Z}[\mathrm{GeV}]$ & $91.1876(21)$ & $\tau_{B_{s H}^{0}}[\mathrm{~s}]$ & $1.620(7) \cdot 10^{-12}$ \\
$m_{\mu}[\mathrm{MeV}]$ & $105.6583745(24)$ & $M_{B_{s}^{0}}[\mathrm{MeV}]$ & $5366.88(14)$ \\
\hline
\end{tabular}

Table 4. The central values are employed in the computations. All values are taken from Particle Data Group (PDG) [20].

$Z$-penguin and box-diagram and it is given by [83]:

$$
\mathcal{L}_{\mathrm{SM}}=-\frac{4 G_{F}}{\sqrt{2}} \frac{\alpha\left(M_{Z}\right)}{2 \pi \sin ^{2} \theta_{\mathrm{W}}} \sum_{\ell=e, \mu, \tau}\left[V_{c s}^{*} V_{c d} X^{\ell}\left(x_{c}\right)+V_{t s}^{*} V_{t d} X^{\ell}\left(x_{t}\right)\right]\left(\overline{s_{L}} \gamma^{\mu} d_{L}\right)\left(\overline{\nu_{\ell L}} \gamma_{\mu} \nu_{\ell L}\right)+\text { h.c. }
$$

$X\left(x_{a}\right)$ are the Inami-Lim function including QCD and electroweak corrections, with $x_{a}=$ $m_{a}^{2} / M_{W}^{2}, a=c, t$. The index $\ell$ denotes the lepton flavor. The dependence on the charged lepton mass is negligible for the top contribution due to $m_{t} \gg m_{\ell}, X^{\ell}\left(x_{t}\right)=X\left(x_{t}\right)$, whereas, being $m_{\tau}$ comparable with $m_{c}$, for the c-quark contribution the box with $\ell=\tau$ gives somewhat different contribution from $\ell=e, \mu[83], X^{e}\left(x_{c}\right)=X^{\mu}\left(x_{c}\right) \neq X^{\tau}\left(x_{c}\right)$. Therefore, an averaged value $\bar{X}=\frac{1}{3}\left(X^{e}\left(x_{c}\right)+X^{\mu}\left(x_{c}\right)+X^{\tau}\left(x_{c}\right)\right)$ is used. Then the effective Lagrangian (4.22) can also be written as:

$$
\begin{aligned}
\mathcal{L}_{\mathrm{SM}} & =-\frac{4 G_{F}}{\sqrt{2}} \mathcal{F}_{K}\left(\overline{s_{L}} \gamma^{\mu} d_{L}\right) \sum_{e, \mu, \tau}\left(\overline{\nu_{\ell L}} \gamma_{\mu} \nu_{\ell L}\right) \\
\mathcal{F}_{K} & =\frac{\alpha\left(M_{Z}\right)}{2 \pi \sin ^{2} \theta_{\mathrm{W}}}\left(V_{t s}^{*} V_{t d} X\left(x_{t}\right)+\left(V_{c s}^{*} V_{c d}\right) \bar{X}\left(x_{c}\right)\right)= \\
& =-\frac{\alpha\left(M_{Z}\right)}{2 \pi \sin ^{2} \theta_{\mathrm{W}}} \lambda^{5}\left(A^{2}(1-\rho-i \eta) X\left(x_{t}\right)+\left(1-\frac{\lambda^{2}}{2}\right) P_{c}(X)\right)
\end{aligned}
$$




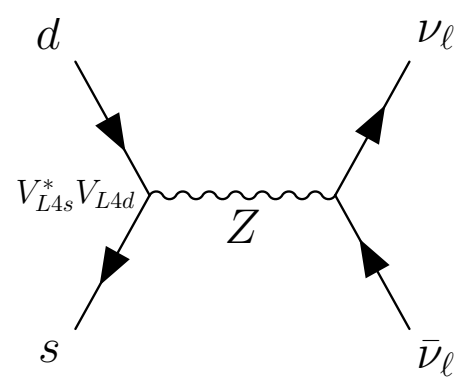

Figure 6. Tree level contribution to the rare kaon decays $K^{+} \rightarrow \pi^{+} \nu \bar{\nu}, K^{0} \rightarrow \pi^{0} \nu \bar{\nu}$ arising from the mixing of the SM families with the extra downtype vectorlike quark.

where $P_{c}(X)=\frac{1}{\lambda^{4}} \bar{X}$, and we used the Wolfenstein parameterization:

$$
\operatorname{Re}\left(V_{c s}^{*} V_{c d}\right)=-\lambda\left(1-\frac{\lambda^{2}}{2}\right), \quad \operatorname{Re}\left(V_{t s}^{*} V_{t d}\right)=-\left(1-\frac{\lambda^{2}}{2}\right) A^{2} \lambda^{5}(1-\rho), \quad \operatorname{Im}\left(V_{t s}^{*} V_{t d}\right)=A^{2} \lambda^{5} \eta .
$$

The top contribution gives $X\left(x_{t}\right)=1.481$ [84] (central value). For the charm contribution, using the values in table 4 , from the formula in ref. [85] we obtain $P_{c}(X) \approx 0.351$. By using $\rho=0.126, \eta=0.392$ and the central values in table 4 we have $\mathcal{F}_{K} \approx(-3.7+i 1.2) \times 10^{-6}$, $\left|\mathcal{F}_{K}\right| \approx 3.9 \times 10^{-6}$. The predicted SM contribution for the branching ratio can be written as:

$$
\operatorname{Br}\left(K^{+} \rightarrow \pi^{+} \nu \bar{\nu}\right)_{\mathrm{SM}} \approx k_{K^{+}}\left|\mathcal{F}_{K}\right|^{2}, \quad k_{K^{+}}=\frac{G_{F}^{2} \tau_{K^{+}} M_{K^{+}}^{5} f_{+}^{2}(0) \mathcal{I}_{\nu}^{+}}{64 \pi^{3}} \approx 5.43
$$

where $f_{+}(0)=0.9699(15)$ is the form factor, $\mathcal{I}_{\nu}^{+}=0.15269$ is the phase space integral [86] and we have used the values in table 4 for the kaon mass and mean life. In this way, we obtain our benchmark value $\operatorname{Br}\left(K^{+} \rightarrow \pi^{+} \nu \bar{\nu}\right)_{\mathrm{SM}} \approx 0.82 \cdot 10^{-10}$, which is within the range of the estimate reported by $\mathrm{PDG} \operatorname{Br}\left(K^{+} \rightarrow \pi^{+} \nu \bar{\nu}\right)_{\mathrm{SM}}=(0.85 \pm 0.05) \cdot 10^{-10}$ [20]. The SM expectation is compatible with the experimental branching ratio [87]:

$$
\operatorname{Br}\left(K^{+} \rightarrow \pi^{+} \nu \bar{\nu}\right)_{\exp }<1.78 \times 10^{-10} \quad(90 \% \text { C.L. })
$$

With future experimental precision, any deviation from the SM prediction of this golden mode branching ratio would indicate towards new physics.

In our BSM scenario with extra vectorlike $b^{\prime}$ quark, the non-diagonal couplings of $Z$-boson with light quarks in eqs. (4.9) induce at tree level the operator with the same structure as the SM one (4.22), as shown in the diagram in figure 6:

$$
\mathcal{L}_{\text {new }}=-\frac{4 G_{F}}{\sqrt{2}} \frac{1}{2} V_{L 4 s}^{*} V_{L 4 d}\left(\overline{s_{L}} \gamma^{\mu} d_{L}\right) \sum_{e, \mu, \tau}\left(\overline{\nu_{\ell \mathrm{L}}} \gamma_{\mu} \nu_{\ell \mathrm{L}}\right)
$$

Thus, the new operator (4.27) contributes to the decay $K^{+} \rightarrow \pi^{+} \nu \bar{\nu}$, in interference with the SM (4.22). The total branching ratio becomes:

$$
\operatorname{Br}\left(K^{+} \rightarrow \pi^{+} \nu \bar{\nu}\right)_{\text {tot }} \approx k_{K^{+}}\left|\frac{1}{2} V_{L 4 s}^{*} V_{L 4 d}+\mathcal{F}_{K}\right|^{2}=\operatorname{Br}\left(K^{+} \rightarrow \pi^{+} \nu \bar{\nu}\right)_{\mathrm{SM}}\left|\frac{\frac{1}{2} V_{L 4 s}^{*} V_{L 4 d}}{\mathcal{F}_{K}}+1\right|^{2}
$$


By using the experimental upper limit (4.26) we have:

$$
\operatorname{Br}\left(K^{+} \rightarrow \pi^{+} \nu \bar{\nu}\right)_{\text {tot }}<1.78 \times 10^{-10}, \quad\left|\frac{\frac{1}{2} V_{L 4 s}^{*} V_{L 4 d}}{\mathcal{F}_{K}}+1\right|<1.5
$$

Figure 11 shows the constraint (4.29) in terms of the modulus and phase of the elements $V_{L 4 d}, V_{L 4 s}$, using $V_{L 4 s}^{*} V_{L 4 d}=\left|V_{L 4 s}^{*} V_{L 4 d}\right| e^{i \delta_{L 21}^{d}}, \delta_{L 21}^{d}=\delta_{L 2}^{d}-\delta_{L 1}^{d}$, from eqs. (4.12) and (4.13). Depending on the unknown relative phase, the limit results in the constraint on the modulus:

$$
\left|V_{L 4 s}^{*} V_{L 4 d}\right|<(0.4 \div 1.9) \times 10^{-5}
$$

As can be seen, in the most conservative case of destructive interference, the new amplitude can be up to 2.5 times the SM amplitude:

$$
\frac{\frac{1}{2}\left|V_{L 4 s}^{*} V_{L 4 d}\right|}{\left|\mathcal{F}_{K}\right|} \lesssim \Delta_{K^{+}}=2.5
$$

In this case we can express the experimental limit in terms of the ratio $\Delta_{K^{+}}$:

$$
\left|V_{L 4 s}^{*} V_{L 4 d}\right| \lesssim 1.9 \cdot 10^{-5}\left[\frac{\Delta_{K^{+}}}{2.5}\right]\left[\frac{\left|\mathcal{F}_{K}\right|}{3.9 \cdot 10^{-6}}\right]
$$

\subsection{2 $\quad K_{\mathrm{L}} \rightarrow \pi^{0} \nu \bar{\nu}$}

The second golden mode is the decay $K_{\mathrm{L}} \rightarrow \pi^{0} \nu \bar{\nu}$. In the Standard Model it is described by the same Lagrangian as in (4.23). However this decay proceeds almost entirely through direct $\mathrm{CP}$ violation, hence it is completely dominated by short-distance loop diagrams with top quark exchanges and the charm contribution can be neglected [88]. In fact, with the phase convention $C\left|K^{0}\right\rangle=\left|\bar{K}^{0}\right\rangle$ (or $C P\left|K^{0}\right\rangle=-\left|\bar{K}^{0}\right\rangle$ ) we have:

$$
\begin{aligned}
\left\langle\pi^{0}\left|(\bar{d} s)_{\mathrm{V}-\mathrm{A}}\right| \bar{K}^{0}\right\rangle & =-\left\langle\pi^{0}\left|(\bar{s} d)_{\mathrm{V}-\mathrm{A}}\right| K^{0}\right\rangle \\
K_{\mathrm{L}} & =\frac{1}{\sqrt{2}}\left[(1+\epsilon) K^{0}+(1-\epsilon) \bar{K}^{0}\right]
\end{aligned}
$$

However, the terms of indirect $(\Delta S=2) \mathrm{CP}$ violation (proportional to $\epsilon$ ) can be neglected in this decay and the dominant contribution comes from the imaginary part of the operator (4.23):

$$
\left\langle\pi^{0} \nu \bar{\nu}\left|\mathcal{L}_{\mathrm{SM}}\right| \frac{K^{0}+\bar{K}^{0}}{\sqrt{2}}\right\rangle=-\frac{4 G_{F}}{\sqrt{2}} \frac{i 2 \operatorname{Im} \mathcal{F}_{K}}{\sqrt{2}}\left\langle\pi^{0}\left|\left(\overline{s_{L}} \gamma^{\mu} d_{L}\right)\right| K^{0}\right\rangle \sum_{\ell=e, \mu, \tau}\left\langle\overline{\nu_{\ell \mathrm{L}}} \gamma_{\mu} \nu_{\ell \mathrm{L}}\right\rangle
$$

The SM predicted branching ratio can be written as:

$$
\operatorname{Br}\left(K_{L} \rightarrow \pi^{0} \nu \bar{\nu}\right)_{\mathrm{SM}} \approx k_{K_{L}}\left|\mathcal{F}_{K}\right|^{2}, \quad k_{K_{L}}=\frac{G_{F}^{2} \tau_{K_{L}} M_{K^{0}}^{5} f_{+}^{2}(0) \mathcal{I}_{\nu}^{L}}{64 \pi^{3}}
$$

where $\mathcal{I}_{\nu}^{+}=0.16043(31)$ is the phase space integral [86]. Putting the values of kaon mass and lifetime (see table 4) we obtain $\operatorname{Br}\left(K^{+} \rightarrow \pi^{+} \nu \bar{\nu}\right)_{\mathrm{SM}} \approx 3.5 \times 10^{-11}$, which practically agrees with the estimate reported by PDG $\operatorname{Br}\left(K_{\mathrm{L}} \rightarrow \pi^{0} \nu \bar{\nu}\right)_{\mathrm{SM}}=(3.0 \pm 0.2) \times 10^{-11}[20]$. 
On the other hand, the experimental limit on this decay is [20]:

$$
\operatorname{Br}\left(K_{\mathrm{L}} \rightarrow \pi^{0} \nu \bar{\nu}\right)_{\exp }<3.0 \times 10^{-9} \quad(90 \% \text { C.L. })
$$

which is two orders of magnitude larger than the SM expectation. Therefore, there is still much room for new physics.

In our BSM scenario with extra vectorlike $b^{\prime}$ quark, the new contribution comes from the imaginary part of the Lagrangian in eq. (4.27) Since the interference term with the SM contribution can be neglected, the experimental limit can be directly applied to the new contribution:

$$
\operatorname{Br}\left(K_{\mathrm{L}} \rightarrow \pi^{0} \nu \bar{\nu}\right)_{\text {new }} \approx k_{K_{L}} \frac{1}{4}\left(\operatorname{Im}\left(V_{L 4 s}^{*} V_{L 4 d}\right)\right)^{2}<\operatorname{Br}_{\exp }
$$

So in the given parameterization (4.12), (4.13) we have:

$$
\left|\operatorname{Im}\left(V_{L 4 s}^{*} V_{L 4 d}\right)\right|=\left|V_{L 4 s}^{*} V_{L 4 d}\right|\left|\sin \left(\delta_{L 21}^{d}\right)\right|<2.2 \times 10^{-5}\left[\frac{\mathrm{Br}_{\exp }}{3.0 \times 10^{-9}}\right]^{\frac{1}{2}}
$$

This condition is shown in figure 11. Then, the present experimental limit allows the new contribution to be one order of magnitude larger than the SM contribution:

$$
\frac{\left|\operatorname{Im}\left(V_{L 4 s}^{*} V_{L 4 d}\right)\right|}{2\left|\operatorname{Im} \mathcal{F}_{K}\right|}<9.3\left[\frac{\mathrm{Br}_{\exp }}{3.0 \cdot 10^{-9}}\right]^{\frac{1}{2}}
$$

So the discovery of the decay $K_{L} \rightarrow \pi^{0} \nu \bar{\nu}$ with branching ratio larger than the SM expectation can be a signal for BSM physics.

\subsection{3 $\quad K_{\mathrm{L}} \rightarrow \pi^{0} e^{+} e^{-}$}

The decay $K_{\mathrm{L}} \rightarrow \pi^{0} e^{+} e^{-}$contains a direct $\mathrm{CP}$ violating contribution, indirect $\mathrm{CP}$ violating contribution, interference between them, and also a small CP conserving contribution [20]. The CP conserving contribution to the amplitude is dominated by a two photon exchange $K_{L} \rightarrow \pi^{0} \gamma \gamma \rightarrow \pi^{0} e^{+} e^{-}$, with both an absorptive and a dispersive part. Using the decay $K_{L} \rightarrow \pi^{0} \gamma \gamma$, it is estimated that the CP-conserving branching ratio is of order $\sim O\left(10^{-13}\right)[20]$. The indirectly $\mathrm{CP}$ violating amplitude also derives from the coupling of leptons to photons, it is given by the long-distance dominated $K_{S} \rightarrow \pi^{0} e^{+} e^{-}$amplitude times the CP parameter $\epsilon_{K}$ [83]. The complete CP-violating contribution to the rate assuming a positive sign for the interference term is estimated to be $\operatorname{Br}\left(K_{\mathrm{L}} \rightarrow \pi^{0} e^{+} e^{-}\right)_{\mathrm{CPV}} \approx(3.1 \pm 0.9) \times 10^{-11}$, where the three contributions from indirect, interference and direct $\mathrm{CP}$ violation are $(1.76,0.9,0.45) \times 10^{-11}$ respectively [20].

Only the direct $\mathrm{CP}$-violating amplitude can be calculated in detail within the SM, since it is short distance dominated. The relevant operator contains the vector part of the hadronic current and both axial and vector component of the leptonic current, and it is given by:

$$
\mathcal{L}_{\mathrm{SM}, \mathrm{SD}}=-\frac{G_{F} \alpha\left(M_{Z}\right)}{\sqrt{2} 2 \pi} V_{t s}^{*} V_{t d}\left(\bar{s} \gamma_{\mu} d\right)\left(\tilde{y}_{V}\left(\bar{e} \gamma^{\mu} e\right)+\tilde{y}_{A}\left(\bar{e} \gamma^{\mu} \gamma^{5} e\right)\right)+\text { h.c. }
$$




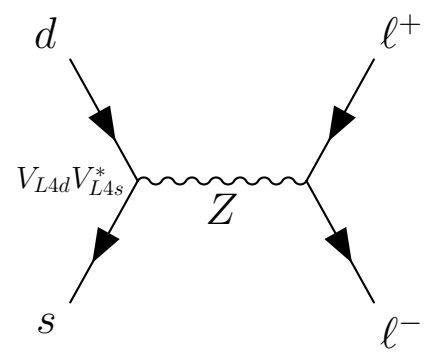

Figure 7. Tree level contribution to the rare kaon decays $K^{0} \rightarrow \pi^{0} \ell^{+} \ell^{-}, K^{0} \rightarrow \mu^{+} \mu^{-}(\ell=e, \mu)$ arising from the mixing of the SM families with the extra downtype vector-like quark.

where $\tilde{y}_{V, A}$ are a combination of Inami-Lim functions of box and $Z$ and $\gamma$ penguin diagrams, as defined in ref. [89]. The direct CP-violating branching ratio in the SM can be written as:

$$
\begin{aligned}
\operatorname{Br}\left(K_{\mathrm{L}} \rightarrow \pi^{0} e^{+} e^{-}\right)_{\mathrm{SM}, \mathrm{SD}} & =k_{K e}\left(\frac{G_{F} \alpha\left(M_{Z}\right)}{\sqrt{2} 2 \pi}\right)^{2}\left(\frac{2 \operatorname{Im} V_{t s}^{*} V_{t d}}{\sqrt{2}}\right)^{2}\left(\tilde{y}_{A}^{2}+\tilde{y}_{V}^{2}\right) \\
k_{K e} & =\frac{1}{2} \frac{M_{K^{0}}^{5} \tau_{K_{L}} f_{+}^{2}(0) \mathcal{I}_{e}}{192 \pi^{3}}
\end{aligned}
$$

where $\mathcal{I}_{e}=0.16043(31)$ [86] is the phase space integral.

The new effective Lagrangian contributing to this decay (as shown in figure 7) is:

$$
\mathcal{L}_{\text {new }}=-\frac{G_{F}}{\sqrt{2}} V_{L 4 s}^{*} V_{L 4 d}\left(\bar{s} \gamma^{\mu} d\right)\left[g_{V} \bar{e} \gamma_{\mu} e+g_{A} \bar{e} \gamma_{\mu} \gamma_{5} e\right]+\text { h.c. }
$$

where $g_{A}=\frac{1}{2}, g_{V}=-\frac{1}{2}+2 \sin ^{2} \theta_{\mathrm{W}} \approx-0.038$. The experimental limit on this decay is [20]:

$$
\operatorname{Br}\left(K_{\mathrm{L}} \rightarrow \pi^{0} e^{+} e^{-}\right)_{\exp }<2.8 \times 10^{-10} \quad(90 \% \text { C.L. })
$$

which is one order of magnitude bigger than the SM expectation. Then, we can consider the SM contribution as negligible and let the new contribution to be the dominant one, imposing a limit directly on the new contribution:

$$
\operatorname{Br}\left(K_{\mathrm{L}} \rightarrow \pi^{0} e^{+} e^{-}\right)_{\text {new }}=k_{K e} G_{F}^{2}\left[\operatorname{Im}\left(V_{L 4 s} V_{L 4 d}^{*}\right)\right]^{2}\left[g_{V}^{2}+g_{A}^{2}\right]<\operatorname{Br}_{\exp }
$$

In the given parameterization (4.12), (4.13) then:

$$
\left|\operatorname{Im}\left(V_{L 4 s} V_{L 4 d}^{*}\right)\right|=\left|V_{L 4 s} V_{L 4 d}^{*}\right|\left|\sin \left(\delta_{L 12}^{d}\right)\right|<1.7 \times 10^{-5}\left[\frac{\mathrm{Br}_{\exp }}{2.8 \cdot 10^{-10}}\right]^{\frac{1}{2}}
$$

where the central values of all the quantities have been used. Also the decay $K_{L} \rightarrow \pi^{0} \mu^{+} \mu^{-}$ gives a comparable constraint, being the experimental limit $\operatorname{Br}\left(K_{\mathrm{L}} \rightarrow \pi^{0} \mu^{+} \mu^{-}\right)_{\exp }<3.8 \times$ $10^{-10}$ (90\% C.L.), to be compared with the SM expectation $\operatorname{Br}\left(K_{\mathrm{L}} \rightarrow \pi^{0} \mu^{+} \mu^{-}\right)=(1.5 \pm$ $0.3) \times 10^{-11}$ (assuming positive interference between the direct- and indirect-CP violating components) [20]. 


\subsection{4 $\quad K_{\mathrm{L}} \rightarrow \mu^{+} \mu^{-}$}

The rare decay $K_{\mathrm{L}} \rightarrow \mu^{+} \mu^{-}$is a $\mathrm{CP}$ conserving decay. Its short-distance part is given by $Z$-penguins and box diagrams. However, this decay is dominated by a long-distance contribution from a two-photon intermediate state. In fact, the full branching ratio can be written as [83]:

$$
\operatorname{Br}\left(K_{\mathrm{L}} \rightarrow \mu^{+} \mu^{-}\right)=|\operatorname{Re} A|^{2}+|\operatorname{Im} A|^{2}, \quad \operatorname{Re} A=A_{\mathrm{SD}}+A_{\mathrm{LD}}
$$

with $\operatorname{Re} A$ and $\operatorname{Im} A$ denoting the dispersive and absorptive contributions, respectively. The absorptive (imaginary) part of the long-distance component is determined by the measured rate for $K_{L} \rightarrow \gamma \gamma$ to be $\operatorname{Br}_{\text {abs }}\left(K_{L} \rightarrow \mu^{+} \mu^{-}\right)=(6.64 \pm 0.07) \cdot 10^{-9}$ and it almost completely saturates the observed rate $\operatorname{Br}\left(K_{\mathrm{L}} \rightarrow \mu^{+} \mu^{-}\right)_{\exp }=(6.84 \pm 0.11) \cdot 10^{-9}$ [20]. The real part of the long-distance amplitude cannot be derived directly from experiment. However in ref. [90] it is estimated an upper bound on the short distance contribution:

$$
\operatorname{Br}\left(K_{\mathrm{L}} \rightarrow \mu^{+} \mu^{-}\right)_{\mathrm{SD}}<2.5 \cdot 10^{-9}
$$

As shown below, the $\mathrm{SM}$ prediction for the short distance contribution results: $\operatorname{Br}\left(K_{\mathrm{L}} \rightarrow\right.$ $\left.\mu^{+} \mu^{-}\right)_{\mathrm{SD}, \mathrm{SM}} \approx 0.9 \cdot 10^{-9}$ [89]. Then the condition (4.49) provides a constraint on new physics scenarios.

Since only the axial component of both hadronic and lepton current contributes to the decay, the effective Lagrangian describing the short distance contribution to the decay in the SM can be written as [83]:

$$
\begin{aligned}
\mathcal{L}_{\mathrm{SM}, \mathrm{SD}} & =\frac{G_{F}}{\sqrt{2}} \frac{\alpha\left(M_{Z}\right)}{2 \pi \sin ^{2} \theta_{\mathrm{W}}}\left(V_{c s}^{*} V_{c d} Y\left(x_{c}\right)+V_{t s}^{*} V_{t d} Y\left(x_{t}\right)\right)\left(\bar{s} \gamma^{\mu} \gamma_{5} d\right)\left(\bar{\mu} \gamma_{\mu} \gamma_{5} \mu\right)+\text { h.c. }= \\
& =\frac{G_{F}}{\sqrt{2}} \mathcal{F}_{L 2}\left(\bar{s} \gamma^{\mu} \gamma_{5} d\right)\left(\bar{\mu} \gamma_{\mu} \gamma_{5} \mu\right)+\text { h.c. }
\end{aligned}
$$

$Y\left(x_{a}\right), x_{a}=m_{a}^{2} / M_{W}^{2}$, are the Inami-Lim functions including QCD and electroweak corrections, whose leading order term is a linear combination of the axial components of $Z$-penguins and box-diagrams. Numerically $Y\left(x_{c}\right) \approx 3.3 \times 10^{-4}, Y\left(x_{t}\right) \approx 0.97$ [83]. In eq. (4.50) we defined the constant $\mathcal{F}_{L 2} \approx(-2.1+i 0.78) \times 10^{-6}$.

In the scenario with extra isosinglet quark, the non-diagonal couplings of $Z$-boson with SM families due to the mixing of light quarks with the $b^{\prime}$-quark leads to the Lagrangian analogous to (4.44), contributing to the decay $K_{\mathrm{L}} \rightarrow \mu^{+} \mu^{-}$at tree level (figure 7 ):

$$
\mathcal{L}_{\text {new }}=\frac{G_{F}}{\sqrt{2}} V_{L 4 s}^{*} V_{L 4 d}\left(\bar{s} \gamma_{\mu} \gamma_{5} d\right) \frac{1}{2}\left(\bar{\mu} \gamma^{\mu} \gamma_{5} \mu\right)+\text { h.c. }
$$

By using again the phase convention $C\left|K^{0}\right\rangle=+\left|\bar{K}^{0}\right\rangle$ we have:

$$
\left\langle 0\left|\bar{d} \gamma_{\mu} \gamma_{5} s\right| \bar{K}^{0}\right\rangle=+\left\langle 0\left|\bar{s} \gamma_{\mu} \gamma_{5} d\right| K^{0}\right\rangle
$$

and neglecting indirect $\mathrm{CP}$ violation:

$$
K_{L} \approx K_{2}=\frac{1}{\sqrt{2}}\left(K^{0}+\bar{K}^{0}\right)
$$


Then we get:

$$
\begin{aligned}
\left\langle\mu^{+} \mu^{-}\left|\mathcal{L}_{\mathrm{SM}, \mathrm{SD}}\right| K_{L}\right\rangle & =\frac{G_{F}}{\sqrt{2}} \frac{2 \operatorname{Re}\left(\mathcal{F}_{L 2}\right)}{\sqrt{2}}\left\langle 0\left|\bar{s} \gamma_{\mu} \gamma_{5} d\right| K^{0}\right\rangle\left[\bar{u}\left(p^{(\mu)}\right) \gamma^{\mu} \gamma_{5} v\left(p^{(\bar{\mu})}\right)\right] \\
\left\langle\mu^{+} \mu^{-}\left|\mathcal{L}_{\text {new }}\right| K_{L}\right\rangle & =\frac{G_{F}}{\sqrt{2}} \frac{\operatorname{Re}\left(V_{L 4 s}^{*} V_{L 4 d}\right)}{\sqrt{2}}\left\langle 0\left|\bar{s} \gamma_{\mu} \gamma_{5} d\right| K^{0}\right\rangle\left[\bar{u}\left(p^{(\mu)}\right) \gamma^{\mu} \gamma_{5} v\left(p^{(\bar{\mu})}\right)\right]
\end{aligned}
$$

Then we can define the branching ratio given by the amplitude of the short distance contribution:

$$
\operatorname{Br}\left(K_{\mathrm{L}} \rightarrow \mu^{+} \mu^{-}\right)_{\mathrm{SD}}=k\left[\operatorname{Re}\left(2 \mathcal{F}_{L 2}+V_{L 4 s}^{*} V_{L 4 d}\right)\right]^{2}, \quad k=\frac{\operatorname{Br}\left(K^{+} \rightarrow \mu^{+} \nu_{\mu}\right) \tau_{K_{L}} M_{K^{0}} \sqrt{1-4 \frac{m_{\mu}^{2}}{M_{K^{0}}^{2}}}}{\tau\left(K^{+}\right) M_{K^{+}}\left(1-\frac{m_{\mu}^{2}}{M_{K^{+}}^{2}}\right)^{2}\left|V_{u s}\right|^{2}}
$$

where we have used $\left\langle 0\left|\bar{s} \gamma_{\mu} \gamma_{5} d\right| K^{0}\right\rangle=i p_{\mu} f_{K}, \operatorname{Br}\left(K^{+} \rightarrow \mu^{+} \nu_{\mu}\right)=0.6356(11)$ and $\left|V_{u s}\right|=$ 0.2252. In absence of new physics, the short distance contribution coincides with the SM expectation $\operatorname{Br}\left(K_{\mathrm{L}} \rightarrow \mu^{+} \mu^{-}\right)_{\mathrm{SD}, \mathrm{SM}}$.

By using the upper bound in eq. (4.49) on the branching ratio (4.56) we have:

$$
\left|\operatorname{Re}\left(2 \mathcal{F}_{L 2}+V_{L 4 s}^{*} V_{L 4 d}\right)\right|<6.9 \times 10^{-6}
$$

This constraint is shown in figure 11 in terms of the modulus $\left|V_{L 4 s}^{*} V_{L 4 d}\right|$ and relative phase $\delta_{L 21}^{d}$, as parameterized in (4.12), (4.13). In terms of the ratio between the new amplitude (4.51) and the SM amplitude (4.50), eq. (4.57) implies:

$$
\left|1+\frac{\operatorname{Re}\left(V_{L 4 s}^{*} V_{L 4 d}\right)}{2 \operatorname{Re}\left(\mathcal{F}_{L 2}\right)}\right|<1.7, \quad 0.7<\frac{\left|\operatorname{Re}\left(V_{L 4 s}^{*} V_{L 4 d}\right)\right|}{2\left|\operatorname{Re} \mathcal{F}_{L 2}\right|}<2.7
$$

or, by using $\operatorname{Re}\left(V_{L 4 s}^{*} V_{L 4 d}\right)=\left|V_{L 4 s}^{*} V_{L 4 d}\right| \cos \delta_{L 21}^{d}$ we have:

$$
-0.28 \times 10^{-5}<\left|V_{L 4 s}^{*} V_{L 4 d}\right| \cos \delta_{L 21}^{d}<1.11 \times 10^{-5},
$$

where we used the Wolfenstein parameterization (4.24).

\subsection{5 $\quad K_{\mathrm{S}} \rightarrow \mu^{+} \mu^{-}$}

The effective Lagrangian (4.50) gives the short distance contribution to the decay $K_{\mathrm{S}} \rightarrow \mu^{+} \mu^{-}$:

$$
\operatorname{Br}\left(K_{\mathrm{S}} \rightarrow \mu^{+} \mu^{-}\right)_{\mathrm{SD}, \mathrm{SM}}=4 k_{K_{S}}\left[\operatorname{Im}\left(\mathcal{F}_{L 2}\right)\right]^{2}
$$

where $k_{K_{S}}$ is the same as $k$ in eq. (4.56) with the change $\tau_{K_{L}} \rightarrow \tau_{K_{S}}$. It is obtained $\operatorname{Br}\left(K_{\mathrm{S}} \rightarrow \mu^{+} \mu^{-}\right)_{\mathrm{SD}} \approx 0.2 \cdot 10^{-12}$. There are also long distance contributions arising from the two photon intermediate state which result in the rate $\operatorname{Br}\left(K_{\mathrm{S}} \rightarrow \mu^{+} \mu^{-}\right)_{\mathrm{LD}} \approx 5.1 \cdot 10^{-12}$ [91]. The experimental upper limit for this decay was found by the LHCb collaboration [92]:

$$
\operatorname{Br}\left(K_{\mathrm{S}} \rightarrow \mu^{+} \mu^{-}\right)_{\exp }<2.1 \times 10^{-10} \quad(90 \% \text { C.L. })
$$




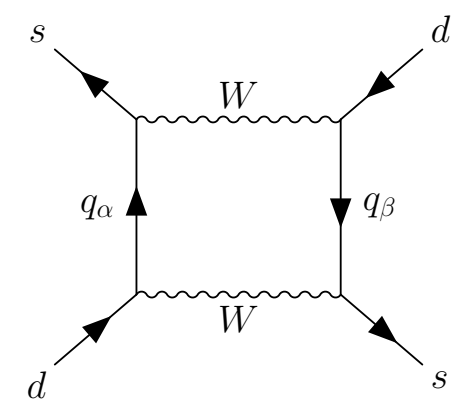

Figure 8. Box diagram for the transition $K^{0} \rightarrow \bar{K}^{0}$ in the SM, $q_{\alpha}, q_{\beta}=u, c, t$.

Then, an extra contribution is allowed to be higher than both the short and long-distance contributions to the decay, so we can impose an upper limit on the new contribution arising in the scenario with the extra isosinglet. The new decay channel is described by the effective Lagrangian (4.51) and gives the rate:

$$
\operatorname{Br}\left(K_{\mathrm{S}} \rightarrow \mu^{+} \mu^{-}\right)_{\text {new }}=k_{K_{S}}\left[\operatorname{Im}\left(V_{L 4 s}^{*} V_{L 4 d}\right)\right]^{2}<\operatorname{Br}_{\text {exp }}
$$

We have:

$$
\left|\operatorname{Im}\left(V_{L 4 s}^{*} V_{L 4 d}\right)\right|=\left|V_{L 4 s}^{*} V_{L 4 d}\right|\left|\sin \delta_{L 21}^{d}\right|<4.8 \times 10^{-5}\left[\frac{\mathrm{Br}_{\exp }}{2.1 \cdot 10^{-10}}\right]^{1 / 2}
$$

where we used the parameterization in eqs. (4.12), (4.13).

\subsection{Limits from neutral mesons systems}

\subsection{1 $K^{0}-\bar{K}^{0}$ mixing}

In the SM the short-distance contribution to the transition $K^{0}(d \bar{s}) \leftrightarrow \bar{K}^{0}(\bar{d} s)$ arises from weak box diagrams (figure 8). The effective Lagrangian describing this contribution is given by:

$$
\mathcal{L}_{\Delta S=2}^{\mathrm{SM}}=-\frac{G_{F}^{2} m_{W}^{2}}{4 \pi^{2}}\left[\lambda_{c}^{2} S_{0}\left(x_{c}\right)+\lambda_{t}^{2} S_{0}\left(x_{t}\right)+2 \lambda_{c} \lambda_{t} S_{0}\left(x_{c}, x_{t}\right)\right]\left(\overline{s_{L}} \gamma^{\mu} d_{L}\right)^{2}+\text { h.c. }
$$

where $\lambda_{a}=V_{a s}^{*} V_{a d}, x_{a}=\frac{m_{a}^{2}}{m_{W}^{2}}$ and $S_{0}\left(x_{i}\right)$ are the Inami-Lim functions [93]:

$$
\begin{aligned}
& S_{0}(x)=x\left(\frac{4-11 x+x^{2}}{4(1-x)^{2}}-\frac{3 x^{2} \ln x}{2(1-x)^{3}}\right) \\
& S_{0}\left(x_{j}, x_{k}\right)=x_{j} x_{k}[\left(\frac{1}{4}-\frac{3}{2\left(x_{j}-1\right)}-\frac{3}{4\left(x_{j}-1\right)^{2}}\right) \frac{\log x_{j}}{x_{j}-x_{k}}+ \\
&\left(\frac{1}{4}-\frac{3}{2\left(x_{k}-1\right)}-\frac{3}{4\left(x_{k}-1\right)^{2}}\right) \frac{\log x_{k}}{x_{k}-x_{j}} \\
&\left.-\frac{3}{4\left(x_{j}-1\right)\left(x_{k}-1\right)}\right]
\end{aligned}
$$


The weak short-distance contribution to the mass splitting $\Delta m_{K}=m_{K_{L}}-m_{K_{S}}$ and the CP-violating parameter $\epsilon_{K}$ are described by the off-diagonal term $M_{12}$ of the mass matrix of neutral kaons, which is given by:

$$
M_{12}=-\frac{1}{2 m_{K^{0}}}\left\langle K^{0}\left|\mathcal{L}_{\Delta S=2}\right| \bar{K}^{0}\right\rangle
$$

In the SM it reads [96]:

$$
M_{12}^{\mathrm{SM}}=\frac{G_{F}^{2} m_{W}^{2}}{12 \pi^{2}}\left(\eta_{1} \lambda_{c}^{* 2} S_{0}\left(x_{c}\right)+\eta_{2} \lambda_{t}^{* 2} S_{0}\left(x_{t}\right)+2 \eta_{3} \lambda_{c}^{*} \lambda_{t}^{*} S_{0}\left(x_{c}, x_{t}\right)\right) f_{K}^{2} m_{K^{0}} B_{K}
$$

where $f_{K}$ is the kaon decay constant, which can be estimated in lattice QCD to be $f_{K}=$ $155.7(0.7) \mathrm{MeV}$ [18], $m_{K^{0}}$ is the neutral kaons mass and we also added the factors $\eta_{1}=$ $1.38 \pm 0.20, \eta_{2}=0.57 \pm 0.01, \eta_{3}=0.47 \pm 0.04$ which describe short-distance QCD effects [83]. The factor $B_{K}$ is the correction to the vacuum insertion approximation (VIA) which is estimated from lattice QCD calculations, giving $B_{K}=0.7625(97)$ [18].

The modulus $\left|M_{12}\right|$ and the imaginary part $\operatorname{Im} M_{12}$ respectively describe short-distance contributions in the mass splitting and CP-violation in $\bar{K}^{0} \rightarrow K^{0}$ transition. In the SM, in the standard parameterization of $V_{\mathrm{CKM}}$ we have:

$$
\begin{gathered}
\left|M_{12}^{\mathrm{SM}}\right| \approx\left|\operatorname{Re} M_{12}^{\mathrm{SM}}\right| \approx \frac{G_{F}^{2} m_{W}^{2}}{12 \pi^{2}} \eta_{1}\left|\lambda_{c}^{2}\right| S_{0}\left(x_{c}\right) f_{K}^{2} m_{K^{0}} B_{K} \approx \frac{G_{F}^{2}}{12 \pi^{2}} \eta_{1}\left|\lambda_{c}^{2}\right| m_{c}^{2} f_{K}^{2} m_{K^{0}} B_{K} \\
\operatorname{Im} M_{12}^{\mathrm{SM}}=-\frac{G_{F}^{2} m_{W}^{2}}{6 \pi^{2}} f_{K}^{2} m_{K^{0}} B_{K}[ \\
\eta_{1} \operatorname{Re}\left(\lambda_{c}\right) \operatorname{Im}\left(\lambda_{c}\right) S_{0}\left(x_{c}\right)+ \\
\left.+\left(\eta_{2} \operatorname{Re}\left(\lambda_{t}\right) S_{0}\left(x_{t}\right)+\eta_{3} \operatorname{Re}\left(\lambda_{c}\right) S_{0}\left(x_{c}, x_{t}\right)\right) \operatorname{Im}\left(\lambda_{t}\right)\right]
\end{gathered}
$$

The mass difference between mass eigenstates is given by [83]:

$$
\Delta m_{K} \approx 2\left|M_{12}\right|+\Delta m_{\mathrm{LD}}
$$

where $\Delta m_{\mathrm{LD}}$ is the long-distance contribution. Although efforts are made, the longdistance contribution is still difficult to evaluate [94, 95]. Nevertheless, the short distance contribution $\Delta m_{K} \approx 2\left|M_{12}^{\mathrm{SM}}\right| \simeq 2.2 \cdot 10^{-15} \mathrm{GeV}$ gives a dominant contribution to the experimentally measured value [20]:

$$
\Delta M_{K}=(3.484 \pm 0.006) \times 10^{-15} \mathrm{GeV}
$$

The CP violation is parameterized by $\epsilon_{K}$, which, with the phase choice $C P\left|K^{0}\right\rangle=-\left|\bar{K}^{0}\right\rangle$, is almost determined by short distance physics [96]:

$$
\left|\epsilon_{K}\right| \approx \frac{\left|\operatorname{Im}\left[M_{12}\right]\right|}{\sqrt{2} \Delta m_{K}}
$$

in the standard parameterization of $V_{\mathrm{CKM}}$. From experimental data it is obtained [20]:

$$
\left|\epsilon_{K}\right|=(2.228 \pm 0.011) \times 10^{-3}
$$

In our BSM scenario with extra vectorlike $b^{\prime}$ quark, the non-diagonal couplings of $Z$ and Higgs bosons with light quarks in eqs. (4.9), (4.21) induce the same operator as in (4.64), 

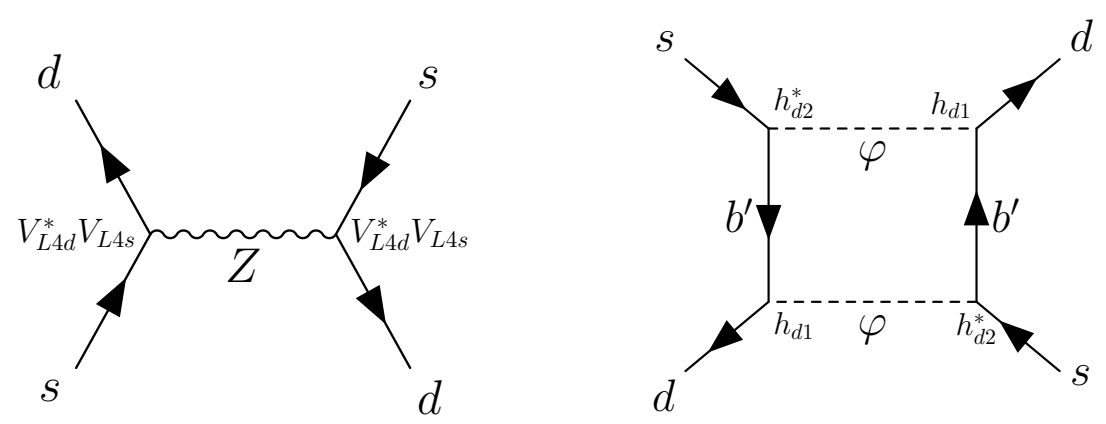

Figure 9. Contributions to $\bar{K}^{0}-K^{0}$ transition due to the insertion of the additional vectorlike down-type weak isosinglet couple $b_{L}^{\prime}, b_{R}^{\prime}$ in quark mixing.

both at tree level and loop level, interfering with the SM in the transition $\bar{K}^{0} \leftrightarrow K^{0}$, as shown in figure 9.

The new tree level contribution translates into the effective Lagrangian:

$$
\mathcal{L}_{\Delta S=2}^{\text {tree }}=-\frac{G_{F}}{\sqrt{2}}\left(V_{L 4 d}^{*} V_{L 4 s}\right)^{2}\left(\overline{d_{L}} \gamma^{\mu} s_{L}\right)^{2}+\text { h.c. }
$$

However if $M_{b^{\prime}}$ is of order few $\mathrm{TeV}$, the loop contribution to $\bar{K}^{0}-K^{0}$ mixing becomes important. In particular, for $M_{b^{\prime}}=3.1 \mathrm{TeV}$ the effective operator for the box diagram with Higgs and $Z$ bosons exchange in figure 9 gives the same contribution as the effective operator of the tree level diagram. Moreover the box diagram contribution grows as $\propto M_{b^{\prime}}^{2}$. In fact, the effective Lagrangian for the loop contribution is:

$$
\mathcal{L}_{\Delta S=2}^{\text {box }} \approx-\frac{\left(h_{d 1} h_{d 2}^{*}\right)^{2}}{128 \pi^{2} M_{b^{\prime}}^{2}}\left(\overline{d_{L}} \gamma^{\mu} s_{L}\right)^{2}+\text { h.c. }
$$

where $h_{d i}$ are the Yukawa couplings defined in eq. (4.1). However, we are interested in fixing a value for the elements of the mixing matrix rather than setting the Yukawas $h_{d i}$. Then, it is convenient to express the Lagrangian explicitly in terms of the mixing elements. From eqs. (4.13), (4.15), we have:

$$
h_{d 1} \approx-V_{L 4 d}^{*} \frac{M_{b^{\prime}}}{v_{w}}, \quad h_{d 2} \approx-V_{L 4 s}^{*} \frac{M_{b^{\prime}}}{v_{w}} .
$$

Then, using $G_{F} / \sqrt{2}=1 / 4 v_{w}^{2}$, the Lagrangian can be written as:

$$
\mathcal{L}_{\Delta S=2}^{\text {box }} \approx-\frac{G_{F}^{2} M_{b^{\prime}}^{2}}{16 \pi^{2}}\left(V_{L 4 d}^{*} V_{L 4 s}\right)^{2}\left(\overline{d_{L}} \gamma^{\mu} s_{L}\right)^{2}+\text { h.c. }
$$

Hence, the complete new contribution is:

$$
\begin{aligned}
\mathcal{L}_{\Delta S=2}^{\text {new }} & =\mathcal{L}_{\Delta S=2}^{\text {tree }}+\mathcal{L}_{\Delta S=2}^{\text {box }} \approx \\
& \approx-\frac{G_{F}}{\sqrt{2}}\left(V_{L 4 d}^{*} V_{L 4 s}\right)^{2}\left(1+\frac{G_{F} M_{b^{\prime}}^{2}}{8 \sqrt{2} \pi^{2}}\right)\left(\overline{d_{L}} \gamma^{\mu} s_{L}\right)^{2}+\text { h.c. }= \\
& =-\frac{G_{F}}{\sqrt{2}}\left(V_{L 4 d}^{*} V_{L 4 s}\right)^{2} f\left(M_{b^{\prime}}\right)\left(\overline{d_{L}} \gamma^{\mu} s_{L}\right)^{2}+\text { h.c. } \\
f\left(M_{b^{\prime}}\right) & =1+\left(\frac{M_{b^{\prime}}}{3.1 \mathrm{TeV}}\right)^{2}
\end{aligned}
$$


where we defined the function $f\left(M_{b^{\prime}}\right)$ of the mass of the extra quark $b^{\prime}$. From the Lagrangian (4.79), there is an additive contribution $M_{12}^{\text {new }}$ to the mixing mass $M_{12}$ (see eq. (4.67)), participating both in the mass splitting and CP-violating effects. Then, the new contribution is constrained by both $\mathrm{CP}$-conserving and $\mathrm{CP}$-violating observables.

Regarding the CP-conserving part, which originates the mass splitting $\Delta m_{K}$, we can confront the moduli of the two components of the mixing mass and impose that the new contribution $\left|M_{12}^{\text {new }}\right|$ is less than a fraction $\Delta_{K}$ of the short-distance SM contribution $\left|M_{12}^{\mathrm{SM}}\right|$ :

$$
\left|M_{12}^{\text {new }}\right|<\left|M_{12}^{\mathrm{SM}}\right| \Delta_{K}
$$

where we defined real and positive $\Delta_{K}$. This is analogous to comparing the modulus of the coefficients of the effective operators in eqs. (4.64) and (4.79). We evaluate the constraint at leading order of both SM and new physics contributions, neglecting QCD corrections. Hence, eq. (4.81) corresponds to:

$$
\frac{G_{F}}{\sqrt{2}} f\left(M_{b^{\prime}}\right)\left|V_{L 4 d}^{*} V_{L 4 s}\right|^{2}<\frac{G_{F}^{2} m_{c}^{2}}{4 \pi^{2}}\left|\lambda_{c}^{2}\right| \Delta_{K}
$$

eq. (4.81) can also be translated into a constraint on the scale of the new contribution:

$$
\frac{G_{F}}{\sqrt{2}} f\left(M_{b^{\prime}}\right)\left|\left(V_{L 4 d}^{*} V_{L 4 s}\right)^{2}\right|<\frac{1}{\Lambda_{s d}^{2}}, \quad \Delta_{K}=\left(\frac{1.9 \cdot 10^{3} \mathrm{TeV}}{\Lambda_{d s}}\right)^{2}
$$

where we defined the minimum allowed equivalent scale $\Lambda_{s d}^{2}$ of the new contribution. We estimate the constraint with $\Delta_{K}=1$, that is by imposing the condition $\left|M_{12}^{\text {new }}\right|<\left|M_{12}^{\mathrm{SM}}\right|$, meaning that the additional processes contribute to $\Delta m_{K}$ at most as the SM short-distance contribution. Anyway, we leave $\Delta_{K}$ as free parameter in next equations so to allow to reevaluate the results with a different constraint. Then, using eqs. (4.12), (4.13), from the condition (4.82) we have:

$$
\left|V_{L 4 d}^{*} V_{L 4 s}\right|<1.7 \times 10^{-4}\left[\frac{f(1 \mathrm{TeV})}{f\left(M_{b^{\prime}}\right)}\right]^{1 / 2}\left[\Delta_{K}\right]^{1 / 2}
$$

where we evaluated the constraint for the benchmark value $M_{b^{\prime}}=1 \mathrm{TeV}$.

Regarding the contribution to the CP-violating parameter $\epsilon_{K}$, the imaginary part of the new contribution to the mixing mass $\operatorname{Im} M_{12}^{\text {new }}$ can be constrained to be a fraction $\Delta_{\epsilon_{K}}$ of the SM contribution:

$$
\left|\operatorname{Im} M_{12}^{\text {new }}\right|<\left|\operatorname{Im} M_{12}^{\mathrm{SM}}\right| \Delta_{\epsilon_{K}},
$$

where we defined real and positive $\Delta_{\epsilon_{K}}$. At leading order of the new physics contribution, eq. (4.85) is equivalent to comparing the magnitude of the imaginary part of operators (4.64) and (4.79):

$$
\begin{aligned}
\frac{G_{F}}{\sqrt{2}} f\left(M_{b^{\prime}}\right)\left|\operatorname{Im}\left[\left(V_{L 4 d}^{*} V_{L 4 s}\right)^{2}\right]\right|< & \frac{G_{F}^{2} m_{W}^{2}}{4 \pi^{2}} 2 \mid \eta_{1} \operatorname{Re}\left(\lambda_{c}\right) \operatorname{Im}\left(\lambda_{c}\right) S_{0}\left(x_{c}\right)+ \\
& +\left(\eta_{2} \operatorname{Re}\left(\lambda_{t}\right) S_{0}\left(x_{t}\right)+\eta_{3} \operatorname{Re}\left(\lambda_{c}\right) S_{0}\left(x_{c}, x_{t}\right)\right) \operatorname{Im}\left(\lambda_{t}\right) \mid \Delta_{\epsilon_{K}}
\end{aligned}
$$


eq. (4.86) can also be translated into a constraint on the scale of the new contribution:

$$
\frac{G_{F}}{\sqrt{2}} f\left(M_{b^{\prime}}\right)\left|\operatorname{Im}\left[\left(V_{L 4 d}^{*} V_{L 4 s}\right)^{2}\right]\right|<\frac{1}{\Lambda_{s d, \operatorname{Im}}^{2}}, \quad \Delta_{\epsilon_{K}}=\left(\frac{1.6 \cdot 10^{4} \mathrm{TeV}}{\Lambda_{d s, \operatorname{Im}}}\right)^{2}
$$

where we defined the minimum allowed equivalent scale $\Lambda_{s d, \text { Im }}^{2}$ of the new contribution. The numerical value is obtained by using the Wolfenstein parameterization as in eq. (4.24), with $\operatorname{Im}\left(V_{c s}^{*} V_{c d}\right)=-A^{2} \lambda^{5} \eta$. We make an estimation choosing $\Delta_{\epsilon_{K}}=0.4$, corresponding to $\Lambda_{d s, \operatorname{Im}}=2.5 \cdot 10^{4} \mathrm{TeV}$. Using eqs. (4.12) and (4.13), from the condition (4.86) we have:

$$
\left|V_{L 4 d}^{*} V_{L 4 s}\right| \sqrt{\left|\sin \left[2\left(\delta_{L 12}^{d}\right)\right]\right|}<1.3 \cdot 10^{-5}\left[\frac{f(1 \mathrm{TeV})}{f\left(M_{b^{\prime}}\right)}\right]^{1 / 2}\left[\frac{\Delta_{\epsilon_{K}}}{0.4}\right]^{1 / 2}
$$

where we evaluated the constraint for the benchmark value $M_{b^{\prime}}=1 \mathrm{TeV}$. The constraints are also shown in figure 11 for $M_{b^{\prime}}=1 \mathrm{TeV}$, in terms of the modulus and relative phase of the elements $V_{L 4 d}^{*} V_{L 4 s}$.

Let us elaborate a little more about the two contributions to $\mathcal{L}_{\Delta S=2}^{\text {new }}$ from the tree and box diagrams. The tree level operator (4.79) can be written in terms of the Yukawa constants $h_{d i}$, in order to be compared with the operator (4.76). Using $G_{F} / \sqrt{2}=1 / 4 v_{w}^{2}$ and eq. (4.77), we have:

$$
\mathcal{L}_{\Delta S=2}^{\text {tree }}=-\frac{\left(h_{d 1} h_{d 2}^{*}\right)^{2}}{4} \frac{v_{w}^{2}}{M_{b^{\prime}}^{4}}\left(\overline{d_{L}} \gamma^{\mu} s_{L}\right)^{2}+\text { h.c. }
$$

which parametrically is a factor $v_{w}^{2} / M_{b^{\prime}}^{2}$ less than the box operator (4.76). That is why, after compensating the loop factor, for $M_{b^{\prime}}>3.1 \mathrm{TeV}$ the box contribution becomes comparable or more important than the tree level contribution.

\subsection{2 $\quad B_{d, s}^{0}-\bar{B}_{d, s}^{0}$ mixing}

In the SM the dominant contribution to $B_{d}^{0}(\bar{b} d)-\bar{B}_{d}^{0}(\bar{d} b)$ and $B_{s}^{0}(\bar{b} s)-\bar{B}_{s}^{0}(\bar{s} b)$ mixings comes from box-diagrams with internal top-quark, while the charm quark and the mixed topcharm contributions are entirely negligible. The effective Lagrangians for $B_{d, s}^{0}-\bar{B}_{d, s}^{0}$ mixings are [83]:

$$
\mathcal{L}_{\Delta B(d)=2}^{\mathrm{SM}}=-\frac{G_{F}^{2}}{4 \pi^{2}} m_{W}^{2} S_{0}\left(x_{t}\right)\left(V_{t b}^{*} V_{t d}\right)^{2}\left(\overline{b_{L}} \gamma_{\mu} d_{L}\right)^{2}+\text { h.c. }
$$

where $S_{0}(x)$ is the Inami-Lim function (4.65), and analogously for $B_{s}^{0}$ system with the substitution $d \rightarrow s$. Then, analogously to the kaons system,

$$
\begin{aligned}
2 M_{B_{d, s}^{0}} M_{12}^{(d, s) *} & =\left\langle\bar{B}_{d, s}^{0}\left|-\mathcal{L}_{\Delta B(d, s)=2}\right| B_{d, s}^{0}\right\rangle \\
\Delta M_{d, s} & =2\left|M_{12}^{(d, s)}\right|
\end{aligned}
$$

In the SM:

$$
\Delta M_{d, s}^{\mathrm{SM}}=\frac{G_{F}^{2} m_{W}^{2}}{6 \pi^{2}} f_{B_{d, s}}^{2} m_{B_{d, s}} B_{B_{d, s}}\left|\left(V_{t b} V_{t d / s}^{*}\right)^{2}\right| \eta_{B} S_{0}\left(x_{t}\right)
$$


where $\eta_{B}$ is the QCD factor, $\eta_{B}=0.551$ [83], and the factors $B_{B_{d, s}}$ are the corrective factors to the vacuum insertion approximation. From lattice QCD calculations: $f_{B_{d}} \sqrt{B_{B_{d}}}=$ $225(9) \mathrm{MeV}, f_{B_{s}} \sqrt{B_{B_{s}}}=274(8) \mathrm{MeV}$ [18]. In contrast to $\Delta m_{K}$, long distance contributions are estimated to be very small in neutral B meson systems, and $\Delta M_{d, s}$ is very well approximated by the relevant box diagrams [83]. The experimental results are [20]:

$$
\begin{aligned}
& \Delta M_{d \exp }=(3.334 \pm 0.013) \cdot 10^{-13} \mathrm{GeV} \\
& \Delta M_{s \exp }=(1.1683 \pm 0.0013) \cdot 10^{-11} \mathrm{GeV}
\end{aligned}
$$

In our BSM scenario with extra vectorlike $b^{\prime}$ quark, the non-diagonal couplings of $Z$ boson and Higgs boson with light quarks in eqs. (4.9), (4.21) induce the operator with the same structure as in (4.90), interfering with the SM in the transition $\bar{B}_{d, s}^{0} \leftrightarrow B_{d, s}^{0}$. The new contributions at tree level and loop level are analogous to the operators giving $K^{0}$ mixing (4.75), (4.78), with diagrams analogous to the ones in figure 9. Then, analogously to the neutral kaons system, the new contribution to neutral B-mesons mixing is:

$$
\begin{aligned}
\mathcal{L}_{\Delta B(d)=2}^{\text {new }} & \approx-\frac{G_{F}}{\sqrt{2}}\left(V_{L 4 d}^{*} V_{L 4 b}\right)^{2} f\left(M_{b^{\prime}}\right)\left(\overline{d_{L}} \gamma^{\mu} b_{L}\right)^{2}+\text { h.c. } \\
\mathcal{L}_{\Delta B(s)=2}^{\text {new }} & \approx-\frac{G_{F}}{\sqrt{2}}\left(V_{L 4 s}^{*} V_{L 4 b}\right)^{2} f\left(M_{b^{\prime}}\right)\left(\overline{s_{L}} \gamma^{\mu} b_{L}\right)^{2}+\text { h.c. }
\end{aligned}
$$

where $f\left(M_{b^{\prime}}\right)$ is defined in eq. (4.80). From the Lagrangians (4.96), (4.97), there is an additive contribution $M_{12}^{\text {new }}$ to the mixing mass $M_{12}^{(d, s)}$ (4.91), and thus to the mass difference $\Delta M_{d, s}$ (4.92). We can constrain the new contribution to be less than a fraction $\Delta_{B_{(d, s)}}$ of the SM contribution $\Delta M_{d, s}^{\mathrm{SM}}=2\left|M_{12}^{(d, s) \mathrm{SM}}\right|$ given in eq. (4.93). This is analogous to comparing the coefficients of the effective operators in eqs. (4.96), (4.97) with the SM ones (4.90):

$$
\frac{G_{F}}{\sqrt{2}} f\left(M_{b^{\prime}}\right)\left|V_{L 4 d / s}^{*} V_{L 4 b}\right|^{2}<\frac{G_{F}^{2}}{4 \pi^{2}} m_{W}^{2}\left|V_{t d / s}\right|^{2} S\left(x_{t}\right) \cdot \Delta_{B_{d / s}}=\frac{1}{\Lambda_{b d / s}^{2}}
$$

where we evaluate the constraint at leading order of both SM and new physics contributions, neglecting QCD corrections. We use $\Delta_{B_{(d, s)}}=0.3$ as a benchmark value, corresponding to the scales $\Lambda_{b d}=1.0 \cdot 10^{3} \mathrm{TeV}, \Lambda_{b s}=2.1 \cdot 10^{2} \mathrm{TeV}$. Then we obtain:

$$
\begin{aligned}
& \left|V_{L 4 d}^{*} V_{L 4 b}\right|<3.3 \times 10^{-4}\left[\frac{f(1 \mathrm{TeV})}{f\left(M_{b^{\prime}}\right)}\right]^{1 / 2}\left[\frac{\Delta_{B_{d}}}{0.3}\right]^{1 / 2} \\
& \left|V_{L 4 s}^{*} V_{L 4 b}\right|<1.6 \times 10^{-3}\left[\frac{f(1 \mathrm{TeV})}{f\left(M_{b^{\prime}}\right)}\right]^{1 / 2}\left[\frac{\Delta_{B_{s}}}{0.3}\right]^{1 / 2}
\end{aligned}
$$

\subsection{3 $\quad D^{0}-\bar{D}^{0}$ mixing}

In the SM $D^{0}(c \bar{u})-\bar{D}^{0}(\bar{c} u)$ mixing receives contributions from box diagrams, dipenguin diagrams and from long-distance effects [96]. In box diagrams only the internal strange and down quarks contribute effectively. Differently from the case of kaons and B-mesons, the masses of $s$ and $d$ are small compared to the mass of the external $c$ quarks, which then 


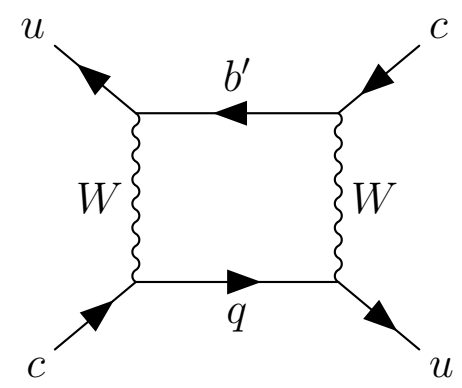

Figure 10. New contribution to $D^{0}-\bar{D}^{0} \operatorname{mixing}, q=d, s, b, b^{\prime}$.

cannot be neglected and an extra operator appears in the effective Lagrangian. In VIA approximation, the box contribution results $\left|M_{12}\right| \sim 10^{-17} \div 10^{-16} \mathrm{GeV}$ [96]. Also dipenguin diagrams can give a contribution not much smaller than box diagrams [97]. Long-distance effects are expected to be large [98]. However, since their contribution is non-perturbative and difficult to compute, there is room for new physics, which in principle can be the dominant contribution to the mass difference $\Delta m_{D}$ in $D^{0}$ system. In fact, a nonzero mass difference in the $D^{0}$ meson system was recently observed by the LHCb collaboration [99]:

$$
\Delta m_{D \exp }=(6.4 \pm 0.9) \times 10^{-15} \mathrm{GeV}
$$

which allows values two orders of magnitude bigger than the SM short-distance expectation.

In the scenario with extra down-type quark, the additional box diagrams with internal $b^{\prime}$ quarks contribute to $D$ mesons mixing, as shown in figure 10 . The corresponding effective Lagrangian is:

$$
\mathcal{L}_{\Delta C=2}^{\text {new }} \approx-\frac{G_{F}^{2} m_{W}^{2}}{4 \pi^{2}}\left(V_{u b^{\prime}} V_{c b^{\prime}}^{*}\right)^{2} S_{0}\left(x_{b^{\prime}}\right)\left(\overline{u_{L}} \gamma^{\mu} c_{L}\right)^{2}+\text { h.c. }
$$

with $x_{b^{\prime}}=\frac{M_{b^{\prime}}^{2}}{m_{W}^{2}}$ (taking $M_{b^{\prime}} \simeq 1 \mathrm{TeV}$, the two mixed contributions would be competitive only if $\left|V_{u b^{\prime}} V_{c b^{\prime}}^{*}\right|<2 \cdot 10^{-7}$, but we are considering scenarios with larger mixings). Since the new effective operator (4.102) originates from charged currents, this process involves directly the elements $V_{u b^{\prime}}, V_{c b^{\prime}}$ of the enlarged CKM matrix $\tilde{V}_{\mathrm{CKM}}$ (4.6). This operator gives an additional contribution $M_{12 \text { new }}^{D}$ to the mixing mass and thus contributes to the mass difference $\Delta m_{D}$ :

$$
\Delta m_{D \text { new }}=2\left|M_{12 \text { new }}^{D}\right| \simeq \frac{G_{F}^{2} m_{W}^{2}}{6 \pi^{2}}\left(V_{u b^{\prime}} V_{c b^{\prime}}^{*}\right)^{2} S_{0}\left(x_{b^{\prime}}\right) f_{D}^{2} m_{D^{0}}
$$

where $f_{D}=212.0 \pm 0.7 \mathrm{MeV}[18]$ is the decay constant. Since we do not know long distance contributions, the new contribution can be regarded as the dominant one, and we can think that the new operator can account up to the entire mass difference in D-mesons system. Then, a rough estimate can be made by imposing on $\Delta m_{D \text { new }}$ to stay within twice the error-bar of the experimental value (4.101):

$$
\begin{aligned}
& \Delta m_{D \text { new }}<8.2 \times 10^{-15} \mathrm{GeV} \\
& \left|V_{u b^{\prime}} V_{c b^{\prime}}^{*}\right|<3.9 \times 10^{-4}\left[\frac{\Delta m_{D \exp }}{8.2 \cdot 10^{-15}}\right]^{1 / 2}\left[\frac{1 \mathrm{TeV}}{M_{b^{\prime}}}\right]
\end{aligned}
$$

The last scaling in eq. (4.105) holds since $S_{0}\left(x_{b^{\prime}}\right) \sim \frac{1}{4} x_{b^{\prime}}$ for $M_{b^{\prime}} \gtrsim 2 \mathrm{TeV}$. 


\subsection{Limits from rare $B$ mesons decays}

\subsection{1 $B_{d, s}^{0} \rightarrow \mu^{+} \mu^{-}$}

The decays $B_{d, s}^{0} \rightarrow \ell^{+} \ell^{-}, \ell=e, \mu, \tau$, are dominated by the $Z$-penguin and box diagrams involving top quark exchanges. The charm contributions are fully negligible here and the effective Lagrangian in the SM is [89]:

$$
\mathcal{L}_{\mathrm{SM}}=\frac{G_{F}^{2} m_{W}^{2}}{2 \pi^{2}} V_{t b}^{*} V_{t q} Y\left(x_{t}\right)\left(\bar{b} \gamma^{\mu} \gamma^{5} q\right)\left(\bar{\ell} \gamma_{\mu} \gamma_{5} \ell\right)
$$

with $q=d, s . Y\left(x_{t}\right)$ is the Inami-Lim function, including QCD and electroweak corrections, which at leading order is a linear combination of Z-penguin and box diagrams, the same as in eq. (4.50). Here we use $Y\left(x_{t}\right)=0.935$ from ref. [100], inserting the values in table 4 .

The non-diagonal couplings of $Z$-boson with SM families lead to the same extra tree level contributions as in eqs. (4.120), (4.121). In this case only the axial part of both quark and leptons is involved:

$$
\mathcal{L}_{\text {new }}=\frac{G_{F}}{\sqrt{2}} \frac{1}{2} V_{L 4 b}^{*} V_{L 4 q}\left(\bar{b} \gamma^{\mu} \gamma^{5} q\right)\left(\bar{\ell} \gamma_{\mu} \gamma_{5} \ell\right)
$$

for $q=d, s$. Considering the case $\ell=\mu$, the branching ratios of the decays $B_{d, s}^{0} \rightarrow \mu^{+} \mu^{-}$ would be:

$$
\operatorname{Br}\left(B_{d / s}^{0} \rightarrow \mu^{+} \mu^{-}\right)_{\text {tot }}=k_{d / s} \frac{G_{F}^{2}}{2}\left|\frac{\alpha}{2 \pi \sin ^{2} \theta_{W}} Y\left(x_{t}\right) V_{t b}^{*} V_{t d / s}+\frac{1}{2} V_{L 4 b}^{*} V_{L 4 d / s}\right|^{2}
$$

where $B_{q}$ is the flavour eigenstate $(\bar{b} q), f_{B_{s}}=230.3(1.3) \mathrm{MeV}, f_{B_{d}}=190.0(1.3) \mathrm{MeV}[18]$ are the decay constants defined by $\left\langle 0\left|\bar{b} \gamma_{\mu} \gamma_{5} q\right| B_{q}^{0}(p)\right\rangle=i p_{\mu} f_{B_{q}}$, and:

$$
k_{d}=\tau_{B_{d}^{0}} \frac{1}{2 \pi} f_{B_{d}}^{2} m_{\mu}^{2} M_{B_{d}^{0}} \sqrt{1-4 m_{\mu}^{2} / M_{B_{d}^{0}}^{2}}, \quad k_{s}=\tau_{B_{s H}^{0}} \frac{1}{2 \pi} f_{B_{s}}^{2} m_{\mu}^{2} M_{B_{s}^{0}} \sqrt{1-4 m_{\mu}^{2} / M_{B_{s}^{0}}^{2}}
$$

In absence of new physics, the branching ratios (4.108) give the SM expectations: $\operatorname{Br}\left(B_{d}^{0} \rightarrow \mu^{+} \mu^{-}\right)_{\mathrm{SM}} \approx 8.6 \times 10^{-11}, \operatorname{Br}\left(B_{s}^{0} \rightarrow \mu^{+} \mu^{-}\right)_{\mathrm{SM}} \approx 3.2 \times 10^{-9}$, obtained using the central values of the quantities in table 4 .

The experimental branching ratio of the decay $B_{s}^{0} \rightarrow \mu^{+} \mu^{-}$is [20]:

$$
\operatorname{Br}\left(B_{s}^{0} \rightarrow \mu^{+} \mu^{-}\right)_{\exp }=(2.9 \pm 0.4) \times 10^{-9}
$$

which is in agreement with the SM expectation. Regarding the decay $B_{d}^{0} \rightarrow \mu^{+} \mu^{-}$, the experimental limit is [101]:

$$
\operatorname{Br}\left(B_{d}^{0} \rightarrow \mu^{+} \mu^{-}\right)_{\exp }<2.1 \times 10^{-10} \quad 95 \% \text { C.L. }
$$

Then, we should set a limit on the decay rates (4.108), in order to not contradict experimental results. At $95 \%$ C.L. we can take:

$$
\begin{array}{r}
\operatorname{Br}\left(B_{d}^{0} \rightarrow \mu^{+} \mu^{-}\right)_{\text {tot }}<2.1 \times 10^{-10} \\
2.1 \times 10^{-9}<\operatorname{Br}\left(B_{s}^{0} \rightarrow \mu^{+} \mu^{-}\right)_{\text {tot }}<3.7 \times 10^{-9}
\end{array}
$$


Depending on the relative unknown phase of the mixing elements $V_{L 4 b}, V_{L 4 d / s}\left(\delta_{L 32}^{d}\right.$ and $\delta_{L 31}^{d}$ in terms of the parameterization $\left.(4.12),(4.13)\right)$, the above upper limits correspond to:

$$
\begin{aligned}
& \left|V_{L 4 b}^{*} V_{L 4 d}\right|<(0.4 \div 2.2) \times 10^{-4} \\
& \left|V_{L 4 b}^{*} V_{L 4 s}\right|<(0.3 \div 8.1) \times 10^{-4}
\end{aligned}
$$

We can express the limits (4.112), (4.113) in terms of comparison between the two effective operators (4.106) and (4.107). By defining:

$$
\mathcal{F}_{B_{q}^{0}}=\frac{\alpha}{2 \pi \sin ^{2} \theta_{W}} Y\left(x_{t}\right) V_{t b}^{*} V_{t q}
$$

the constraint can be written as:

$$
\frac{\frac{1}{2}\left|V_{L 4 b}^{*} V_{L 4 q}\right|}{\left|\mathcal{F}_{B_{q}^{0}}\right|}<\Delta_{B_{q}^{0}}
$$

Then, in the case of destructive interference, the limits (4.114), (4.115) correspond to:

$$
\begin{array}{r}
\left|V_{L 4 b}^{*} V_{L 4 d}\right|<2.2 \times 10^{-4}\left[\frac{\Delta_{B_{d}^{0}}}{2.7}\right]\left[\frac{\mathcal{F}_{B_{d}^{0}}}{4.1 \times 10^{-5}}\right] \\
{\left[\frac{\Delta_{B_{s}^{0}}}{1.8}\right] 7.1 \times 10^{-4}<\left|V_{L 4 b}^{*} V_{L 4 s}\right|<8.1 \times 10^{-4}\left[\frac{\Delta_{B_{s}^{0}}}{2.1}\right]\left[\frac{\mathcal{F}_{B_{s}^{0}}}{2.0 \times 10^{-4}}\right]}
\end{array}
$$

or $\left|V_{L 4 b}^{*} V_{L 4 s}\right|<0.7 \times 10^{-4}\left[\frac{\Delta_{B_{s}^{0}}}{0.2}\right]$ in the second case, for $\delta_{L 31}^{d}=2.72$ and $\delta_{L 32}^{d}=\pi$ respectively.

\subsubsection{Rare semileptonic B decays}

Results on rare B-decays can constrain mixings of the extra vector-like quark with SM families because of the new contributions to FCNC processes involving $b \rightarrow s(d)$ originated at tree level by non-diagonal couplings of $Z$-boson with SM quarks.

The new effective Lagrangian contributing to $b \rightarrow s(d) \ell^{+} \ell^{-}$decays are:

$$
\begin{aligned}
& \mathcal{L}_{\text {new }}=-\frac{4 G_{F}}{\sqrt{2}} V_{L 4 d} V_{L 4 b}^{*}\left(\overline{b_{L}} \gamma^{\mu} d_{L}\right)\left[\left(-\frac{1}{2}+\sin ^{2} \theta_{W}\right)\left(\overline{\ell_{L}} \gamma_{\mu} \ell_{L}\right)+\sin ^{2} \theta_{W}\left(\overline{\ell_{R}} \gamma_{\mu} \ell_{R}\right)\right] \\
& \mathcal{L}_{\text {new }}=-\frac{4 G_{F}}{\sqrt{2}} V_{L 4 b}^{*} V_{L 4 s}\left(\overline{b_{L}} \gamma^{\mu} s_{L}\right)\left[\left(-\frac{1}{2}+\sin ^{2} \theta_{W}\right)\left(\overline{\ell_{L}} \gamma_{\mu} \ell_{L}\right)+\sin ^{2} \theta_{W}\left(\overline{\ell_{R}} \gamma_{\mu} \ell_{R}\right)\right]
\end{aligned}
$$

giving both exclusive decays, such as $B \rightarrow \pi \ell^{+} \ell^{-}$and $B \rightarrow K \ell^{+} \ell^{-}$, and inclusive decays $B \rightarrow X_{s(d)} \ell^{+} \ell^{-}$, where $X_{s(d)}$ stands for anything with a $s(d)$ quark.

As regards exclusive decays $b \rightarrow s \ell^{+} \ell^{-}$, experimental branching fractions are below SM predictions [20]. The decays $B^{0} \rightarrow K^{0 *} \ell^{+} \ell^{-}$and $B^{+} \rightarrow K^{+} \ell^{+} \ell^{-}$are the best studied and tensions with SM were found related to the quantity $P_{5}^{\prime}$ and to lepton universality test [20] (and references therein). As regards inclusive decays, experimental measurements of $B \rightarrow X_{s} \ell^{+} \ell^{-}$were made by both Belle [102] and BaBar [103]. Their data are consistent with SM expectations at $95 \%$ C.L. [104]. In order to estimate the constraint on the mixings $\left|V_{L 4 d} V_{L 4 b}^{*}\right|,\left|V_{L 4 s} V_{L 4 b}^{*}\right|$, we can consider the total branching fraction averaged between electrons and muons, which experimentally results [20]:

$$
\operatorname{Br}\left(B \rightarrow X_{s} \ell^{+} \ell^{-}\right) \exp =(5.8 \pm 1.3) \times 10^{-6}
$$


SM calculations predict $\operatorname{Br}\left(B \rightarrow X_{s} \ell^{+} \ell^{-}\right)_{\mathrm{SM}}=(4.6 \pm 0.8) \times 10^{-6}$ [105]. Let us define the branching ratio which would be given only by the new contribution:

$$
\begin{aligned}
\left|A_{\text {new }}\right|^{2} & =\operatorname{Br}\left(B \rightarrow X_{s} \ell^{+} \ell^{-}\right)_{\text {new }} \simeq \\
& \simeq \operatorname{Br}\left(B \rightarrow X_{c} \ell^{+} \nu_{\ell}\right) \frac{\left|V_{L 4 b}^{*} V_{L 4 s}\right|^{2}\left[\left(-\frac{1}{2}+\sin ^{2} \theta_{W}\right)^{2}+\sin ^{4} \theta_{W}\right]}{\left|V_{c b}\right|^{2}}<\operatorname{Br}\left(B \rightarrow X_{s} \ell^{+} \ell^{-}\right)_{\max }
\end{aligned}
$$

$\operatorname{Br}\left(B \rightarrow X_{s} \ell^{+} \ell^{-}\right)_{\max }$ is defined here as the maximal allowed value for the branching ratio associated to the amplitude of the new contribution alone and we use the experimental branching ratio $\operatorname{Br}\left(B \rightarrow X_{c} \ell^{+} \nu_{\ell}\right)=0.1065(16)$ [20]. Similarly $\left|A_{\mathrm{SM}}\right|^{2}=\operatorname{Br}(B \rightarrow$ $\left.X_{s} \ell^{+} \ell^{-}\right)_{\mathrm{SM}}$. In order to have a rough indication of the constraint on the mixings, we can limit the new prediction with twice the error-bars of the experimental result:

$$
3.25 \times 10^{-6}<\left|A_{\text {new }}+A_{\mathrm{SM}}\right|^{2}<8.35 \times 10^{-6}
$$

Then, we can obtain the least stringent constraint from destructive interference:

$$
\left|V_{L 4 b}^{*} V_{L 4 s}\right|<1.8 \times 10^{-3}\left[\frac{\operatorname{Br}\left(B \rightarrow X_{s} \ell^{+} \ell^{-}\right)_{\max }}{2.5 \times 10^{-5}}\right]^{\frac{1}{2}}
$$

and, for this case of destructive interference, $\left|V_{L 4 b}^{*} V_{L 4 s}\right|>1.4 \times 10^{-3}$.

As regards $b \rightarrow d \ell^{+} \ell^{-}$transitions, the LHCb collaboration measured the branching ratio for the decay $B^{+} \rightarrow \pi^{+} \mu^{+} \mu^{-}[20,106]$ :

$$
\operatorname{Br}\left(B^{+} \rightarrow \pi^{+} \mu^{+} \mu^{-}\right) \exp =(1.78 \pm 0.23) \times 10^{-8}
$$

which agrees with SM predictions, for example $\operatorname{Br}\left(B^{+} \rightarrow \pi^{+} \mu^{+} \mu^{-}\right) \mathrm{SM}=1.88_{-0.21}^{+0.32} \times$ $10^{-8}$ [107], $\operatorname{Br}\left(B^{+} \rightarrow \pi^{+} \mu^{+} \mu^{-}\right)_{\mathrm{SM}}=(2.04 \pm 0.21) \times 10^{-8}$ [108]. Other than that, upper limits on exclusive decays include Belle constraint $\operatorname{Br}\left(B^{+} \rightarrow \pi^{+} \ell^{+} \ell^{-}\right)<4.9 \cdot 10^{-8}$ (90\% C.L.) [109] and BaBar result [110]:

$$
\operatorname{Br}\left(B^{0} \rightarrow \pi^{0} \ell^{+} \ell^{-}\right) \exp <5.3 \cdot 10^{-8} \quad 90 \% \text { C.L. }
$$

$\ell=e$ or $\mu$. The SM expectation for the $B^{0}$ decay can be obtained from the predictions of $B^{+}$ branching ratios by multiplying by the factor $\left(\tau_{B^{0}} / \tau_{B^{+}}\right) / 2$, where $\tau_{B^{0}} / \tau_{B^{+}}=1.076(4)$ [20]. Let us define as before the decay rate which would arise only from the new contribution:

$$
\left|A_{B^{ \pm, 0} \rightarrow \pi^{ \pm, 0} \ell^{+} \ell^{-}}^{\text {new }}\right|^{2}=\operatorname{Br}\left(B^{ \pm, 0} \rightarrow \pi^{ \pm, 0} \ell^{+} \ell^{-}\right)_{\text {new }} \simeq k_{B^{ \pm, 0}}\left|V_{L 4 d} V_{L 4 b}^{*}\right|^{2}
$$

Then we should have:

$$
\begin{array}{r}
1.3 \times 10^{-8}<\left|A_{B^{ \pm} \rightarrow \pi^{ \pm} \ell^{+} \ell^{-}}^{\text {new }}+A_{B^{ \pm} \rightarrow \pi^{ \pm} \ell^{+} \ell^{-}}^{\text {SM }}\right|^{2}<2.2 \times 10^{-8} \\
\left|A_{B^{0} \rightarrow \pi^{0} \ell^{+} \ell^{-}}^{\text {new }}+A_{B^{0} \rightarrow \pi^{0} \ell^{+} \ell^{-}}^{\text {SM }}\right|^{2}<5.3 \cdot 10^{-8}
\end{array}
$$


where we defined $\left|A_{\mathrm{SM}}\right|^{2}=\mathrm{Br}_{\mathrm{SM}}$. We used the $2 \sigma$ interval of the experimental result (4.126) and the upper limit (4.127). In order to have the least stringent constraint, we can consider the case of destructive interference between the new contribution and the SM one:

$$
\begin{aligned}
& \left|V_{L 4 d} V_{L 4 b}^{*}\right|<2.5 \times 10^{-4}\left[\frac{\operatorname{Br}\left(B^{ \pm} \rightarrow \pi^{ \pm} \ell^{+} \ell^{-}\right)_{\max }}{1.1 \times 10^{-7}}\right]^{\frac{1}{2}} \\
& \left|V_{L 4 d} V_{L 4 b}^{*}\right|<4.2 \times 10^{-4}\left[\frac{\operatorname{Br}\left(B^{0} \rightarrow \pi^{0} \ell^{+} \ell^{-}\right)_{\max }}{1.1 \times 10^{-7}}\right]^{\frac{1}{2}}
\end{aligned}
$$

where $\mathrm{Br}_{\max }$ is defined as in eq. (4.123) as the maximal allowed value for the branching ratio induced by the amplitude of the new contribution alone, and we used

$$
\begin{aligned}
k_{B^{ \pm}} & =2 \frac{\operatorname{Br}\left(B^{ \pm} \rightarrow \pi^{0} \ell^{+} \nu_{\ell}\right)\left[\left(-\frac{1}{2}+\sin ^{2} \theta_{W}\right)^{2}+\sin ^{4} \theta_{W}\right]}{\left|V_{u b}\right|^{2}} \\
k_{B^{0}} & =\frac{1}{2} \frac{\operatorname{Br}\left(B^{0} \rightarrow \pi^{-} \ell^{+} \nu_{\ell}\right)\left[\left(-\frac{1}{2}+\sin ^{2} \theta_{W}\right)^{2}+\sin ^{4} \theta_{W}\right]}{\left|V_{u b}\right|^{2}}
\end{aligned}
$$

with $\operatorname{Br}\left(B^{0} \rightarrow \pi^{-} \ell^{+} \nu_{\ell}\right)=(1.50 \pm 0.06) \times 10^{-4}, \operatorname{Br}\left(B^{ \pm} \rightarrow \pi^{0} \ell^{+} \nu_{\ell}\right)=(7.80 \pm 0.27) \times 10^{-5}$, $\ell=e$ or $\mu$ [20]. Regarding the measured decay $B^{ \pm} \rightarrow \pi^{ \pm} \ell^{+} \ell^{-}$, in this case we also obtain $\left|V_{L 4 b}^{*} V_{L 4 d}\right|>2.2 \times 10^{-4}$.

\subsection{Limits from $Z$-boson physics}

The presence of additional vector-like quarks also affects the diagonal couplings of Z-boson with standard quarks, changing the prediction of many observables related to the $Z$-boson physics e.g. the $Z$ total width $\Gamma_{Z}$, the partial decay width into hadrons $\Gamma(Z \rightarrow$ had $)$, the partial decay widths $R_{c}=\Gamma(c \bar{c}) / \Gamma(Z \rightarrow$ had $), R_{b}=\Gamma(b \bar{b}) / \Gamma(Z \rightarrow$ had $), \Gamma(Z \rightarrow q \bar{q})$, $q=u, d, s, c, b$, etc. Constraints obtained from these quantities are analized in this section. Experimental values and SM predictions are taken from Particle Data Group [20], as reported in table 5 .

The predicted partial decay width of Z-boson decaying into $b \bar{b}$ is $\Gamma(Z \rightarrow b \bar{b})_{\mathrm{SM}} \approx$ $375.75 \mp 0.18 \mathrm{MeV}$, which should be compared to the experimental value $\Gamma(Z \rightarrow b \bar{b})_{\exp } \simeq$ $377.3 \mp 1.2 \mathrm{MeV}$, (using data from PDG [20], as reported in table 5). The SM prediction of the partial decay rate of $Z \rightarrow b \bar{b}$ at tree level is given by:

$$
\Gamma(Z \rightarrow b \bar{b})_{\mathrm{SM}}=\frac{G_{F} M_{Z}^{3}}{\sqrt{2} \pi}\left[\left(-\frac{1}{2}+\frac{1}{3} \sin ^{2} \theta_{W}\right)^{2}+\left(\frac{1}{3} \sin ^{2} \theta_{W}\right)^{2}\right]
$$

In order to compare with the experimental result, we should include QCD corrections, which are given by a multiplicative factor $\approx 1.021[20]$. By inserting the vector-like isosinglet down-quark, the decay rate at tree level changes in:

$$
\Gamma(Z \rightarrow b \bar{b})=\frac{G_{F} M_{Z}^{3}}{\sqrt{2} \pi}\left[\left(-\frac{1}{2}\left(1-\left|V_{L 4 b}\right|^{2}\right)+\frac{1}{3} \sin ^{2} \theta_{W}\right)^{2}+\left(\frac{1}{3} \sin ^{2} \theta_{W}\right)^{2}\right]
$$

which means:

$$
\Gamma(Z \rightarrow b \bar{b})-\Gamma(Z \rightarrow b \bar{b})_{\mathrm{SM}} \approx \frac{G_{F} M_{Z}^{3}}{\sqrt{2} \pi}\left(-\frac{1}{2}+\frac{1}{3} \sin ^{2} \theta_{W}\right)\left|V_{L 4 b}\right|^{2}<0
$$




\begin{tabular}{|lll|}
\hline Quantity & Experimental value & SM prediction \\
\hline$\Gamma_{Z}$ & $2.4952 \pm 0.0023 \mathrm{GeV}$ & $2.4942 \pm 0.0009 \mathrm{GeV}$ \\
$\Gamma($ had $)$ & $1.7444 \pm 0.0020 \mathrm{GeV}$ & $1.7411 \pm 0.0008 \mathrm{GeV}$ \\
$R_{b}$ & $0.21629 \pm 0.00066$ & $0.21581 \pm 0.00002$ \\
$R_{c}$ & $0.1721 \pm 0.0030$ & $0.17221 \pm 0.00003$ \\
$A_{F B}^{(0, b)}$ & $0.0992 \pm 0.0016$ & $0.1030 \pm 0.0002$ \\
$A_{F B}^{(0, c)}$ & $0.0707 \pm 0.0035$ & $0.0736 \pm 0.0002$ \\
$A_{F B}^{(0, s)}$ & $0.0976 \pm 0.0114$ & $0.1031 \pm 0.0002$ \\
$A_{b}$ & $0.923 \pm 0.020$ & 0.9347 \\
$A_{c}$ & $0.670 \pm 0.027$ & $0.6677 \pm 0.0001$ \\
$A_{s}$ & $0.895 \pm 0.091$ & 0.9356 \\
$g_{A V}^{e u}$ & & -0.1888 \\
$g_{A V}^{e d}$ & & 0.3419 \\
$Q_{W}(C s)$ & $-72.82 \pm 0.42$ & $-73.23 \pm 0.01$ \\
$Q_{W}(T \ell)$ & $-116.4 \pm 3.6$ & $-116.87 \pm 0.02$ \\
$g_{A V}^{e p}$ & $-0.0356 \pm 0.0023$ & -0.0357 \\
$g_{A V}^{e n}$ & $0.4927 \pm 0.0031$ & 0.4950 \\
$2 g_{A V}^{e u}-g_{A V}^{e d}$ & $-0.7165 \pm 0.0068$ & -0.7195 \\
\hline
\end{tabular}

Table 5. Values of interest from Particle Data Group [20].

So the prediction for the decay rate is lowered, not going towards the direction of a better agreement with the experimental value. Then, the extra contribution to the rate should be constrained:

$$
\left|\Gamma(Z \rightarrow b \bar{b})-\Gamma(Z \rightarrow b \bar{b})_{\mathrm{SM}}\right|<\Delta \Gamma_{Z b b}
$$

We may choose $\Delta \Gamma_{Z b b}$ so that $\Gamma(Z \rightarrow b \bar{b})_{\text {tot }}$ lays in the $95 \%$ C.L. interval of the experimental value, which implies:

$$
\begin{aligned}
\left|\Gamma(Z \rightarrow b \bar{b})-\Gamma(Z \rightarrow b \bar{b})_{\mathrm{SM}}\right| & <8.6 \times 10^{-4} \mathrm{GeV} \\
\left|V_{L 4 b}\right| & <3.2 \cdot 10^{-2}\left[\frac{\Delta \Gamma_{Z b b}}{8.6 \times 10^{-4} \mathrm{GeV}}\right]^{1 / 2}
\end{aligned}
$$

The SM predictions for the $\mathrm{Z}$ decay rate and partial decay rate into hadrons are [20]:

$$
\Gamma(Z)_{\mathrm{SM}}=2.4942 \pm 0.0009 \mathrm{GeV}, \quad \Gamma(Z \rightarrow \operatorname{hadr})_{\mathrm{SM}}=1.7411 \pm 0.0008 \mathrm{GeV}
$$

to be compared with the experimental results [20]:

$$
\Gamma(Z)_{\exp }=2.4952 \pm 0.0023 \mathrm{GeV}, \quad \Gamma(Z \rightarrow \text { hadr })_{\exp }=1.7444 \pm 0.0020 \mathrm{GeV}
$$

In this BSM scenario, the deviation from the SM expectation of the new predicted $Z$ partial decay rate into hadrons $\Gamma(Z \rightarrow$ had) (which also corresponds to the deviation of the total 
$Z$ decay rate $\Gamma(Z)$ if there are not additional leptons) is:

$$
\begin{aligned}
& \Gamma(Z \rightarrow \text { had })-\Gamma(Z \rightarrow \text { had })_{\mathrm{SM}}=\Gamma(Z)-\Gamma(Z)_{\mathrm{SM}}= \\
& =\frac{G_{F} M_{Z}^{3}}{\sqrt{2} \pi}\left[\sum_{i, j=d, s, b}\left|-\frac{1}{2} \sum_{k=1}^{3} V_{L k i}^{*} V_{L k j}+\frac{1}{3} \sin ^{2} \theta_{W} \delta_{i j}\right|^{2}-3\left(-\frac{1}{2}+\frac{1}{3} \sin ^{2} \theta_{W}\right)^{2}\right] \approx \\
& \approx \frac{G_{F} M_{Z}^{3}}{\sqrt{2} \pi}\left(-\frac{1}{2}+\frac{1}{3} \sin ^{2} \theta_{W}\right)\left(\left|V_{L 4 d}\right|^{2}+\left|V_{L 4 s}\right|^{2}+\left|V_{L 4 b}\right|^{2}\right)<0
\end{aligned}
$$

QED+QCD corrections should also be included, which amount to a multiplicative factor $\approx 1.041$ for $d, s$-quarks and $\approx 1.021$ for $b$-quark (there is additional correction for the bottom quark due to a loop with the $\mathrm{W}$ boson and the top quark) [20]. As shown in eq. (4.143), the prediction for the decay rate is lowered with respect to the SM expectation $\Gamma(Z \rightarrow$ hadr) $)_{\mathrm{SM}}$. Then, since the SM expectation (4.141) is below the experimental result (4.142), the contribution of the extra quarks is not leading towards a better agreement. Therefore, in order to set a constraint on the new expected decay rate, we can impose that the new expectation $\Gamma(Z \rightarrow$ had $)$ should be in the $95 \%$ C.L. interval of the experimental value $\Gamma(Z \rightarrow \text { had })_{\exp }$, using in eq. (4.143) the limit value for the SM prediction $\Gamma(Z \rightarrow \text { had })_{\mathrm{SM}}=$ $1.7419 \mathrm{GeV}$. That gives:

$$
\begin{aligned}
\Gamma(Z \rightarrow \text { had })_{\mathrm{SM}}-\Gamma(Z \rightarrow \mathrm{had}) & <\Delta \Gamma_{Z} \\
\left|V_{L 4 d}\right|^{2}+\left|V_{L 4 s}\right|^{2}+\left|V_{L 4 b}\right|^{2} & <1.7 \times 10^{-3}\left[\frac{\Delta \Gamma_{Z}}{1.4 \times 10^{-3} \mathrm{GeV}}\right]
\end{aligned}
$$

With $\left|V_{L 4 d}\right|=0.03$, the constraint means $\left|V_{L 4 s}\right|^{2}+\left|V_{L 4 b}\right|^{2}<0.0008$, which is satisfied for example if both $\left|V_{L 4 s}\right|,\left|V_{L 4 b}\right|<0.02$. If $V_{L 4 s}=V_{L 4 b}=0$, this constraint implies:

$$
\left|V_{L 4 d}\right|^{2}<1.7 \times 10^{-3}, \quad\left|V_{L 4 d}\right|<0.041
$$

which is extremely close to the value needed to solve the CKM unitarity problem (for example, at $95 \%$ C.L. $\left|V_{L 4 d}\right|=0.043_{-0.015}^{+0.011}$ (4.8) using our conservative averages for $V_{u d}$ and $V_{u s}$ values (2.10), (2.14)). This means that an extra weak singlet could not completely explain the CKM unitarity anomaly with more extreme values of the determinations of CKM elements, as can be seen for example by comparing eq. (4.145) to the needed values $\left|V_{L 4 d}\right|^{2}=\delta_{\mathrm{CKM}}$ displayed in table 2 .

Constraints are expected also from Z-pole asymmetry analyses of $e^{+} e^{-} \rightarrow f f$ processes. In particular, left-right asymmetries $A_{L R}$, forward-backward asymmetries $A_{F B}$ and left-right forward-backward asymmetries $A_{L R F B}$ [111] were measured at LEP. Cross sections for $Z$-boson exchange are usually written in terms of the asymmetry parameters $A_{f}$, $f=e, \mu, \tau, b, c, s, q$, which contain final-state couplings. For example, they are related as $A_{F B}^{(0, f)}=\frac{3}{4} A_{e} A_{f}, A_{L R F B}^{(0, f)}=\frac{3}{4} A_{f}$ (where the superscript 0 indicates the quantity corrected for radiative effects). The presence of an additional isosinglet changes the couplings of quarks with the $Z$ boson as in eq. (4.9). Consequently the predictions for the asymmetries are also changed:

$$
A_{q}=\frac{\left(1-\left|V_{L 4 q}\right|^{2}\right)^{2}-4\left|Q_{q}\right| \bar{s}_{q}^{2}\left(1-\left|V_{L 4 q}\right|^{2}\right)}{\left(1-\left|V_{L 4 q}\right|^{2}\right)^{2}-4\left|Q_{q}\right| \bar{s}_{q}^{2}\left(1-\left|V_{L 4 q}\right|^{2}\right)+8 Q_{q}^{2} \bar{s}_{q}^{4}}
$$


where $\bar{s}_{f}^{2}$ are the effective weak angles which take into account EW radiative corrections. Then, the mixing $\left|V_{L 4 q}\right|$ with the isosinglet quark makes the prediction for $A_{q}$ lower than the SM one. The most precise result for quarks regards the asymmetry for b-quark final state. Taking the data from Particle Data Group [20] (also listed in table 5), in principle the mixing $\left|V_{L 4 b}\right|$ modifies the couplings in the "good" direction with respect to the experimental determinations of both $A_{b}$ and $A_{F B}^{(0, b)}$. However, considering the other constraints from $Z$ decays, a mixing $\left|V_{L 4 b}\right| \lesssim 0.03$ (4.140) would only give a relative change to $A_{b}$ at most of $\sim 0.016 \%$, two orders of magnitude less than the relative experimental error.

\subsection{Summary of experimental limits}

As illustrated in section 2, the analysis of the latest determinations of $V_{u s}$ obtained from kaon decays and the ones of $V_{u d}$ from beta decays results in a deviation from unitarity in the first row of the CKM matrix. The SM unitarity relation (1.3) can be modified into the relation in eq. (4.7) if an extra vector-like down-type quark $d_{L 4}, d_{R 4}$ participates in the mixing with SM families. In section 4 it was shown that a quite large mixing with the first family, $\left|V_{u b^{\prime}}\right| \approx\left|V_{L 4 d}\right| \approx 0.043(7)$ (4.8), is needed in order to explain the data (see also table 2 , where in this case $\delta_{\mathrm{CKM}}=\left|V_{u b^{\prime}}\right|^{2}$ ). Then, we need to verify if such large mixing is compatible with experimental constraints from flavour changing decays and electroweak observables.

In table 6 the relevant constraints extracted in this section are listed.

Limits from each flavour changing kaon process are shown in figure 11, where $V_{L 4 d}$ and $V_{L 4 s}$ are elements of the mixing matrix $V_{L}^{(d)}(4.4)$. In order to not contradict any bound, depending on the relative phase, the product $\left|V_{L 4 d} V_{L 4 s}^{*}\right|$ cannot exceed the limit:

$$
\left|V_{L 4 d} V_{L 4 s}^{*}\right| \lesssim(0.3 \div 1.7) \times 10^{-5}
$$

taking $M_{b^{\prime}}=1 \mathrm{TeV}$. Using the parameterization in eqs. (4.12), (4.13), the maximum and minimum values are obtained for $\delta_{L 21}^{d} \approx 1.6 \pi$ and $\delta_{L 21}^{d}=\pi$ respectively. Taking into account the needed values to recover the unitarity of the CKM matrix in eq. (4.8), we use $\left|V_{u b^{\prime}}\right| \approx\left|V_{L 4 d}\right|=0.03$ as a conservative benchmark value. Then, from eq. (4.147) we have:

$$
\left|V_{L 4 s}\right|<(0.9 \div 5.0) \times 10^{-4}\left[\frac{0.03}{\left|V_{u b^{\prime}}\right|}\right]
$$

Constraints on the value of $\left|V_{t b^{\prime}}\right| \approx\left|V_{L 4 b}\right|$ are obtained from flavour changing B-mesons decays. The present most stringent bound is given by the decay $B^{0} \rightarrow \mu^{+} \mu^{-}$(4.114):

$$
\left|V_{L 4 b}^{*} V_{L 4 d}\right|<(0.4 \div 2.2) \times 10^{-4}, \quad\left|V_{L 4 b}\right|<(1.4 \div 7.4) \times 10^{-3}\left[\frac{0.03}{\left|V_{u b^{\prime}}\right|}\right]
$$

depending on the relative phase $\delta_{L 31}^{d}$, where the maximum and minimum value are obtained respectively for $\delta_{L 31}^{d}=2.72$ and $\delta_{L 31}^{d}=-0.42$.

Then, taking into account that (4.13), (4.15):

$$
V_{L 4 d} \approx-\frac{h_{d 1}^{*} v_{w}}{M_{b^{\prime}}}, \quad V_{L 4 s} \approx-\frac{h_{d 2}^{*} v_{w}}{M_{b^{\prime}}}, \quad V_{L 4 b} \approx-\frac{h_{d 3}^{*} v_{w}}{M_{b^{\prime}}}
$$




\begin{tabular}{|lll|}
\hline Process & Constraint & \\
\hline$K^{+} \rightarrow \pi^{+} \nu \bar{\nu}$ & $\left|V_{L 4 s}^{*} V_{L 4 d}\right|<1.9 \times 10^{-5}$ & $\left|V_{L 4 s}\right|<6.4 \times 10^{-4}\left[\frac{0.03}{\left|V_{u b^{\prime}}\right|}\right]$ \\
$K_{\mathrm{L}} \rightarrow \pi^{0} \nu \bar{\nu}$ & $\left|V_{L 4 s}^{*} V_{L 4 d}\right|\left|\sin \delta_{L 21}^{d}\right|<2.2 \times 10^{-5}$ & $\left|V_{L 4 s}\right|<\frac{7.4 \times 10^{-4}}{\left|\sin \delta_{L 21}^{d}\right|}\left[\frac{0.03}{\left|V_{u b^{\prime}}\right|}\right]$ \\
$K_{\mathrm{L}} \rightarrow \pi^{0} e^{+} e^{-}$ & $\left|V_{L 4 s}^{*} V_{L 4 d}\right|\left|\sin \delta_{L 21}^{d}\right|<1.7 \times 10^{-5}$ & $\left|V_{L 4 s}\right|<\frac{5.5 \times 10^{-4}}{\left|\sin \delta_{L 21}^{d}\right|}\left[\frac{0.03}{\left|V_{u b^{\prime}}\right|}\right]$ \\
$K_{\mathrm{L}} \rightarrow \mu^{+} \mu^{-}$ & $-0.3 \times 10^{-5}<\left|V_{L 4 s}^{*} V_{L 4 d}\right| \cos \delta_{L 21}^{d}<1.1 \times 10^{-5}$ & $\left|V_{L 4 s}\right|<\frac{3.7 \times 10^{-4}}{\left|\cos \delta_{L 21}^{d}\right|}\left[\frac{0.03}{\left|V_{u b^{\prime}}\right|}\right]$ \\
$K_{S} \rightarrow \mu^{+} \mu^{-}$ & $\left|V_{L 4 s}^{*} V_{L 4 d}\right|\left|\sin \delta_{L 21}^{d}\right|<4.8 \times 10^{-5}$ & $\left|V_{L 4 s}\right|<\frac{1.6 \times 10^{-3}}{\left|\sin \delta_{L 21}^{d}\right|}\left[\frac{0.03}{\left|V_{u b^{\prime}}\right|}\right]$ \\
$K^{0}-\bar{K}^{0}$ & $\left|V_{L 4 s}^{*} V_{L 4 d}\right|<1.7 \times 10^{-4}$ & $\left|V_{L 4 s}\right|<5.8 \times 10^{-3}\left[\frac{0.03}{\left|V_{u b^{\prime}}\right|}\right]$ \\
& $\left|V_{L 4 s}^{*} V_{L 4 d}\right| \sqrt{\left|\sin \left(2 \delta_{L 21}^{d}\right)\right|<1.3 \times 10^{-5}}$ & $\left|V_{L 4 s}\right|<\frac{4.4 \times 10^{-4}}{\sqrt{\mid \sin \left(2 \delta_{L 21}^{d}\right)}\left[\frac{0.03}{\left|V_{u b^{\prime}}\right|}\right]}$ \\
\hline$B^{0}-\bar{B}^{0}$ & $\left|V_{L 4 b} V_{L 4 d}^{*}\right|<3.3 \times 10^{-4}$ & $\left|V_{L 4 b}\right|<1.1 \times 10^{-2}\left[\frac{0.03}{\left|V_{u b^{\prime}}\right|}\right]$ \\
$B^{0} \rightarrow \pi^{0} \ell^{+} \ell^{-}$ & $\left|V_{L 4 b} V_{L 4 d}^{*}\right|<4.2 \times 10^{-4}$ & $\left|V_{L 4 b}\right|<1.4 \times 10^{-2}\left[\frac{0.03}{\left|V_{u b^{\prime}}\right|}\right]$ \\
$B^{ \pm} \rightarrow \pi^{ \pm} \ell^{+} \ell^{-}$ & $\left|V_{L 4 b} V_{L 4 d}^{*}\right|<2.5 \times 10^{-4}$ & $\left|V_{L 4 b}\right|<8.4 \times 10^{-3}\left[\frac{0.03}{\left|V_{u b^{\prime}}\right|}\right]$ \\
$B^{0} \rightarrow \mu^{+} \mu^{-}$ & $\left|V_{L 4 b} V_{L 4 d}^{*}\right|<2.2 \times 10^{-4}$ & $\left|V_{L 4 b}\right|<7.4 \times 10^{-3}\left[\frac{0.03}{\left|V_{u b^{\prime}}\right|}\right]$ \\
\hline$B_{s}^{0}-\bar{B}_{s}^{0}$ & $\left|V_{L 4 b} V_{L 4 s}^{*}\right|<1.6 \times 10^{-3}$ & \\
$B \rightarrow X_{s} \ell^{+} \ell^{-}$ & $\left|V_{L 4 b} V_{L 4 s}^{*}\right|<1.8 \times 10^{-3}$ & $\left|V_{c b^{\prime}}\right|<1.3 \times 10^{-2}\left[\frac{0.03}{\left|V_{u b^{\prime}}\right|}\right]$ \\
$B_{s}^{0} \rightarrow \mu^{+} \mu^{-}$ & $\left|V_{L 4 b} V_{L 4 s}^{*}\right|<8.1 \times 10^{-4}$ & \\
\hline$D^{0}-\bar{D}^{0}$ & $\left|V_{u b^{\prime}} V_{c b^{\prime}}^{*}\right|<3.9 \times 10^{-4}\left[\frac{1 \mathrm{TeV}}{M_{b^{\prime}}}\right]$ & \\
\hline$Z \rightarrow b \bar{b}$ & $\left|V_{L 4 b}\right|<3.2 \cdot 10^{-2}$ & \\
\hline$\Gamma_{Z}, Z \rightarrow h a d r$ & $\left|V_{L 4 d}\right|<0.041$ & \\
\hline & & \\
\hline$a b$ &
\end{tabular}

Table 6. Limits on the mixing of the SM three families with a fourth down-type vector-like isosinglet. As in eq. (4.12), $\left(\delta_{L i}^{d}-\delta_{L j}^{d}\right)=\delta_{L i j}^{d}$. Regarding the elements of CKM matrix, $V_{u b^{\prime}} \approx$ $-V_{L 4 d}^{*}, V_{c b^{\prime}} \approx-V_{L 4 d}^{*} V_{c d}-V_{L 4 s}^{*} V_{c s}-V_{L 4 b}^{*} V_{c b}, V_{t b^{\prime}} \approx-V_{L 4 b}^{*}$ as in eqs. (4.18), (4.19), (4.20). For details see the text.

where $h_{d i}$ are the Yukawa couplings defined in eq. (4.1), from eqs. (4.148), (4.149) it follows that the Yukawa couplings $h_{d 2}$ and $h_{d 3}$ should be respectively at least 50 times and 4 times smaller than the coupling of the first family $h_{d 1}$.

As regards the new column of the enlarged CKM matrix (4.6), we have $\left|V_{u b^{\prime}}\right| \approx\left|V_{L 4 d}\right|$, $\left|V_{t b^{\prime}}\right| \approx\left|V_{L 4 b}\right|$ and, from eq. (4.19):

$$
\begin{aligned}
\left|V_{c b^{\prime}}\right| & \approx\left|-V_{L 4 d}^{*} V_{c d}-V_{L 4 s}^{*} V_{c s}-V_{L 4 b}^{*} V_{c b}\right|= \\
& =|| V_{c d}|| V_{u b^{\prime}}|-| V_{L 4 s}\left|e^{i \delta_{L 21}^{d}}-\right| V_{c b}|| V_{L 4 b}\left|e^{i \delta_{L 31}^{d} \mid}\right| \approx\left|V_{c d}\right|\left|V_{u b^{\prime}}\right|
\end{aligned}
$$

where in the last step we have taken into account the constraints (4.148), (4.149). In fact, in order to have the needed value $\left|V_{u b^{\prime}}\right| \approx 0.043$, there is no much room in the parameter space to accommodate the relation (4.151) without contradicting experimental constraints from $D^{0}$ mixing and flavour changing kaon decays, as shown in figure 12 . There, the blue 


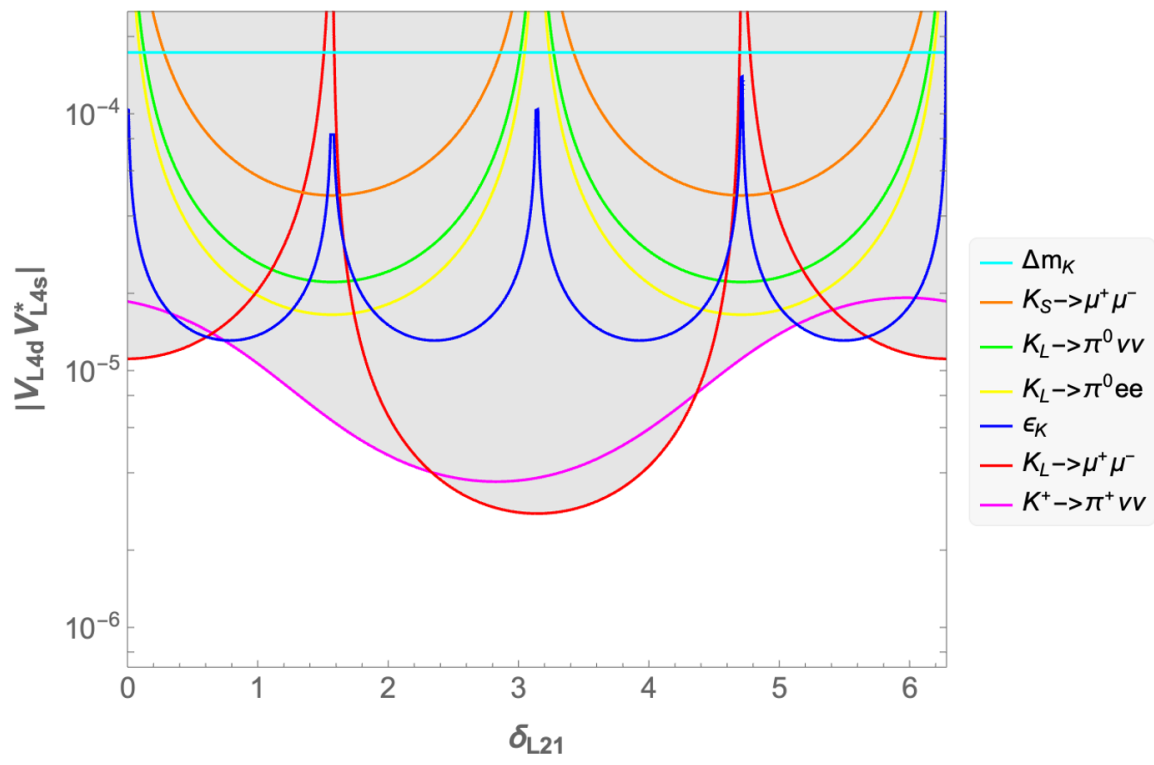

Figure 11. Upper limits obtained from kaon decays and neutral kaon mixing on the product $\left|V_{L 4 s}^{*} V_{L 4 d}\right|$ of the elements of the mixing matrix $V_{L}^{(d)}(4.4)$, as a function of their relative phase $\delta_{L 21}^{d}$.

area is excluded by flavour changing processes involving K-mesons (4.148). We use the indicative upper limit obtained for $\delta_{L 21}^{d}=\operatorname{Arg}\left(V_{L 4 s}^{*} V_{L 4 d}\right)=0,\left|V_{L 4 s}^{*} V_{L 4 d}\right|<1.1 \times 10^{-5}$, which is allowed by every single process for $-3 / 2 \pi \lesssim \delta_{L 21}^{d} \lesssim 0.3 \pi$ (for other choices the constraint should be more stringent, see figure 11). The green region is excluded by the relation in eq. (4.151), for any value of the relative phase $\delta_{L 31}^{d}$ and for the considered values of the relative phase $\delta_{L 21}^{d}$ (other values of $\delta_{L 21}^{d}$ only allow a slightly larger opening angle of the allowed "cone" on the upper side, which does not widen the allowed range of values of $V_{c b^{\prime}}$, because the constraint from kaon physics is more stringent for those other values of the phase $\left.\delta_{L 21}^{d}\right)$. The red area is excluded by the constraints on $\left|V_{c b^{\prime}}\right|$ from $D^{0}$ systems (4.105) with $M_{b^{\prime}}=1 \mathrm{TeV}$ :

$$
\left|V_{u b^{\prime}} V_{c b^{\prime}}^{*}\right|<3.9 \times 10^{-4}\left[\frac{\Delta m_{D \exp }}{8.2 \cdot 10^{-15}}\right]^{1 / 2}\left[\frac{1 \mathrm{TeV}}{M_{b^{\prime}}}\right]
$$

which, together with eq. (4.151) (and flavour changing kaon processes), implies:

$$
\left|V_{u b^{\prime}}\right| \lesssim 0.042\left[\frac{\Delta m_{D \exp }}{8.2 \cdot 10^{-15}}\right]^{1 / 4}\left[\frac{1 \mathrm{TeV}}{M_{b^{\prime}}}\right]^{1 / 2}
$$

which already excludes the more extreme values needed to explain the Cabibbo angle anomaly (for example, at $95 \%$ C.L. $\left|V_{L 4 d}\right|=0.043_{-0.015}^{+0.011}$ (4.8) using our conservative average for $V_{u d}(2.10)$, or see also the needed values $\left|V_{L 4 d}\right|^{2}=\delta_{\text {CKM }}$ displayed in table 2). As discussed in section 4.2.3, the constraint is obtained by allowing the new contribution to the mass difference in $D^{0}$ mesons system to account for the experimental value $\Delta m_{D \text { exp }}$, in the $2 \sigma$ interval. The red dashed lines indicate the boundary of the excluded area under more stringent assumptions. In particular, the new contribution to D-mesons mass difference is limited to be equal to the central experimental value (4.101) (left) and one third of 

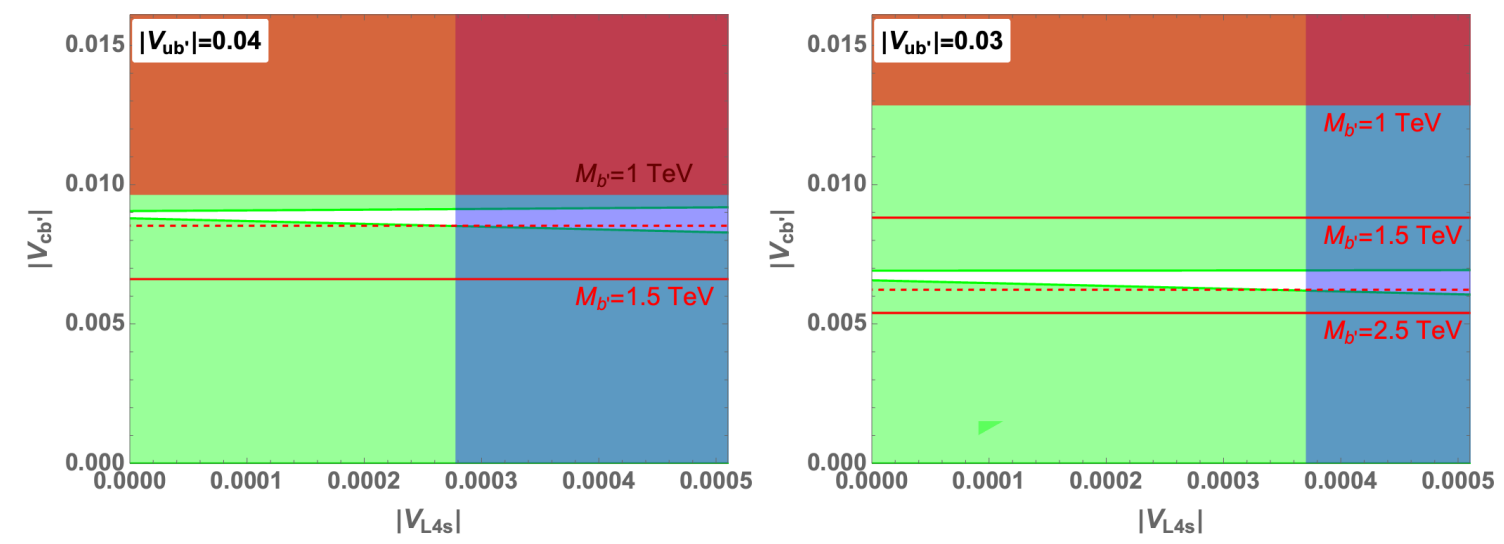

Figure 12. Constraints on $\left|V_{c b^{\prime}}\right|$ and $\left|V_{L 4 s}\right|$. On the left, $\left|V_{u b^{\prime}}\right| \approx\left|V_{L 4 d}\right|=0.04$. The red area is excluded by the constraints on $\left|V_{c b^{\prime}}\right|$ from $D^{0}$ systems, with $M_{b^{\prime}}=1 \mathrm{TeV}$, as reported in table 6 . The red lines show where the boundary shifts if the mass of the extra quark is taken as $M_{b^{\prime}}=1.5$. The red dashed line indicate the boundary of the excluded area if the new contribution to D-mesons mass difference is equal to the central experimental value (4.101). The blue area is excluded by flavour changing processes involving K-mesons, using the result $\left|V_{L 4 s}\right|<3.7 \cdot 10^{-4}$, from figure 11 , eq. (4.148), which is allowed by every single process for $-3 / 2 \pi \lesssim \delta_{L 21}^{d} \lesssim 0.3 \pi$. The green region is excluded by the relation in eq. (4.151), for the considered values of the relative phase $\delta_{L 21}^{d}$. On the right, the same constraints are shown setting $\left|V_{u b^{\prime}}\right| \approx\left|V_{L 4 d}\right|=0.03$. The constraint on $\left|V_{c b^{\prime}}\right|$ from D-mesons mixing (red area) is lowered to the red lines taking the mass of the extra quark as $M_{b^{\prime}}=1.5 \mathrm{TeV}, M_{b^{\prime}}=2.5 \mathrm{TeV}$. The red dashed line indicate the boundary of the excluded area under more stringent assumptions. In particular, the new contribution to D-mesons mass difference is limited as one third of the experimental value (4.101).

the experimental value (right). This means that a deeper knowledge of the long distance contribution (and corresponding sign) can exclude this possibility if it will saturate the experimental value or, viceversa, the extra singlet quark can be thought as a way to explain the value of the mass difference in neutral D-mesons system in case of deviation between experimental and expected value. A narrow allowed region (including $V_{L 4 s}=0$ ) can be found for values $V_{u b^{\prime}}<0.042$. However, in any case, the mass of the extra quark cannot exceed few $\mathrm{TeV}$. In fact, with larger values of $M_{b^{\prime}}$ the constraint from $D^{0}$ mixing becomes more stringent. For example, as shown in figure 12, the allowed region vanishes if $M_{b^{\prime}}=$ $1.5 \mathrm{TeV}$ for $\left|V_{u b^{\prime}}\right|=0.04$, and even with a conservative choice $\left|V_{u b^{\prime}}\right|=0.03$ the allowed area disappear with $b^{\prime}$ mass less than $M_{b^{\prime}}=2.5 \mathrm{TeV}$. Also the constraint from flavour changing processes of kaons shifts towards lower values with larger values of the extra quark mass.

In addition to that, results on $Z$-boson decay rate into hadrons imply that (4.145):

$$
\left|V_{L 4 d}\right|^{2}<1.7 \times 10^{-3}, \quad\left|V_{u b^{\prime}}\right| \approx\left|V_{L 4 d}\right|<0.041
$$

which is also in the range of values needed to solve the CKM unitarity problem (for example, at $95 \%$ C.L. $\left|V_{L 4 d}\right|=0.043_{-0.015}^{+0.011}$. This means that also precision measurements of $\mathrm{Z}$ boson decays can exclude down-type weak singlet as a good solution of the CKM unitarity problem. 
We can also notice that the mixings of $b^{\prime}$ with c-quark and t-quark $\left|V_{c b^{\prime}}\right|,\left|V_{t b^{\prime}}\right|$ should be at least four times smaller than the mixing with the u-quark. Moreover, $\left|V_{u b^{\prime}}\right| \sim 0.03$ is comparable to $\left|V_{c b}\right|$ and ten times larger than $\left|V_{u b}\right|$. However, although it may seem unnatural to expect a larger mixing of the 4th state with the lightest family than with the heavier ones, it cannot be excluded. Moreover, with $\left|V_{u b^{\prime}}\right|=0.03, M_{b^{\prime}} \lesssim 6 \mathrm{TeV}$ would be needed for the perturbativity, assuming $\left|h_{d 1}\right| \lesssim 1$.

\section{Extra up-type isosinglet}

The case of the addition of a vector-like up-type isosinglet couple of quarks $\left(u_{4 L}, u_{4 R}\right)$ is examined in this section. Analogously to the case of the extra down-type quark, there is an additional piece in the Yukawa Lagrangian:

$$
y_{i j}^{u} \tilde{\varphi} \overline{q_{L i}} u_{R j}+y_{i j}^{d} \varphi \overline{q_{L i}} d_{R j}+h_{u i} \tilde{\varphi} \overline{q_{L i}} u_{R 4}+M_{4 u} \overline{u_{L 4}} u_{R 4}+\text { h.c. }
$$

with $i, j=1,2,3$ and $\tilde{\varphi}=i \tau_{2} \varphi^{*}$. Then, the up-type quarks mass matrix looks in a way analogous to the mass matrix in eq. (4.2) (with substitution $d \rightarrow u$ ), and it is similarly diagonalized by two unitary $4 \times 4$ matrices with positive eigenvalues $m_{u, c, t}=y_{u, c, t} v_{w}$ and $M_{t^{\prime}}$. Weak and mass eigenstates and the unitary matrices $V_{L, R}^{(u)}$ can be defined as in eq. (4.4). Since only the three up-type quarks $u_{L 1}, u_{L 2}, u_{L 3}$ couple with $W$-bosons, the Lagrangian for the charged current interaction is:

$$
\begin{aligned}
\mathcal{L}_{\mathrm{cc}} & =\frac{g}{\sqrt{2}}\left(\overline{u_{L 1}} \overline{u_{L 2}} \overline{u_{L 3}}\right) \gamma^{\mu}\left(\begin{array}{c}
d_{L 1} \\
d_{L 2} \\
d_{L 3}
\end{array}\right) W_{\mu}^{+}+\text {h.c. }= \\
& =\frac{g}{\sqrt{2}}\left(\overline{u_{L}} \overline{c_{L}} \overline{t_{L}} \overline{t_{L}^{\prime}}\right) \gamma^{\mu} \tilde{V}_{\mathrm{CKM}}\left(\begin{array}{c}
d_{L} \\
s_{L} \\
b_{L}
\end{array}\right) W_{\mu}^{+}+\text {h.c. }
\end{aligned}
$$

where

$$
\tilde{V}_{\mathrm{CKM}}=\tilde{V}_{L}^{(u) \dagger}=\left(\begin{array}{ccc}
V_{u d} & V_{u s} & V_{u b} \\
V_{c d} & V_{c s} & V_{c b} \\
V_{t d} & V_{t s} & V_{t b} \\
V_{t^{\prime} d} & V_{t^{\prime} s} & V_{t^{\prime} b}
\end{array}\right)
$$

is a $4 \times 3$ matrix, $\tilde{V}_{L}^{(u)}$ is the $3 \times 4$ submatrix of $V_{L}^{(u)}$ without the last row and the unitary $3 \times 3$ matrix diagonalizing the down-type quark mass matrix from the left, $V_{L}^{(d)}$, is taken diagonal. Again $\tilde{V}_{\mathrm{CKM}}$ is not unitary:

$$
\tilde{V}_{\mathrm{CKM}} \tilde{V}_{\mathrm{CKM}}^{\dagger}=\tilde{V}_{L}^{(u) \dagger} \tilde{V}_{L}^{(u)} \neq \mathbf{1}
$$

In particular, for the first row it holds that

$$
\left|V_{u d}\right|^{2}+\left|V_{u s}\right|^{2}+\left|V_{u b}\right|^{2}=\left[\tilde{V}_{\mathrm{CKM}} \tilde{V}_{\mathrm{CKM}}^{\dagger}\right]_{11}=\left[\tilde{V}_{L}^{(u) \dagger} \tilde{V}_{L}^{(u)}\right]_{11}=1-\left|V_{L 4 u}\right|^{2}
$$


which is the same extended unitarity condition as in eqs. (2.1) and (4.7), where $\delta_{\mathrm{CKM}}=$ $\left|V_{L 4 u}\right|^{2}$ and $\left|V_{L 4 u}\right|$ has the same effect on the unitarity of the first row as $\left|V_{u b^{\prime}}\right|$. Then, the analysis of the determinations of $V_{u s}$ obtained from leptonic and semileptonic kaon decays and of $V_{u d}$ from beta decays gives the same result as in section 4, leading to the best fit point in eq. (4.8):

$$
\left|V_{L 4 u}\right|^{2}=1.83(55) \times 10^{-3}, \quad\left|V_{L 4 u}\right|=0.043(7)
$$

which shifts the values of $V_{u s}$ obtained from the three determinations as shown in figure 5 .

The mixing matrix $V_{L}^{(u)}$ induces non-standard couplings of $Z$-boson with the LH up quarks because of different weak isospin couplings, as also described in section 4 . Then, the weak neutral current Lagrangian for up quarks reads:

$$
\mathcal{L}_{\mathrm{nc}}=\frac{g}{\cos \theta_{W}}\left[\frac{1}{2}\left(\overline{u_{L}} \overline{c_{L}} \overline{t_{L}} \overline{t_{L}^{\prime}}\right) \gamma^{\mu} \tilde{V}_{L}^{(u) \dagger} \tilde{V}_{L}^{(u)}\left(\begin{array}{c}
u_{L} \\
c_{L} \\
t_{L} \\
t_{L}^{\prime}
\end{array}\right)-\frac{2}{3} \sin ^{2} \theta_{W}\left(\overline{\mathbf{u}_{L}} \gamma^{\mu} \mathbf{u}_{L}+\overline{\mathbf{u}_{R}} \gamma^{\mu} \mathbf{u}_{R}\right)\right] Z_{\mu}
$$

where $\mathbf{u}$ is the column vector of the four up-type quarks $u, c, t, t^{\prime}$. Then, as shown in eq. (5.7), the non-unitarity of $\tilde{V}_{L}^{(u)}$ is at the origin of non-diagonal couplings with $Z$ boson, which means flavor changing neutral currents (FCNC) at tree level. The weak isospin dependent $Z$ couplings are the same as in eq. (4.10) with substitutions $d \rightarrow u, s \rightarrow c$, $b \rightarrow t$. $V_{L}^{(u)}$ can be parameterized analogously to eqs. (4.11), (4.12). As it will be shown, in order to have $V_{L 4 u} \approx 0.03$, it should be $\left|V_{L 4 c}\right| \lesssim 4.2 \times 10^{-3},\left|V_{t^{\prime} b}\right| \lesssim 8.5 \times 10^{-2}$. Then, as regards the elements of the fourth row of $\tilde{V}_{\mathrm{CKM}}=\tilde{V}_{L}^{(u) \dagger}$, it holds that:

$$
\begin{aligned}
& V_{t^{\prime} d} \approx-V_{L 4 u} \\
& V_{t^{\prime} s} \approx-V_{L 4 u} V_{u s}-V_{L 4 c} V_{c s}-V_{L 4 t} V_{t s} \\
& V_{t^{\prime} b} \approx-V_{L 4 t}
\end{aligned}
$$

with:

$$
\begin{aligned}
V_{L 4 u} & =-\tilde{s}_{L 1}^{u} \approx \frac{h_{u 1}^{*} v_{w}}{M_{t^{\prime}}}, \quad V_{L 4 c} \approx-\tilde{s}_{L 2}^{u} \approx \frac{h_{u 2}^{*} v_{w}}{M_{t^{\prime}}}, \quad V_{L 4 t} \approx-\tilde{s}_{L 3}^{u} \approx \frac{h_{u 3}^{*} v_{w}}{M_{t^{\prime}}} \\
\tilde{s}_{L i}^{u} & =\sin \theta_{L i 4}^{u} e^{i \delta_{L i}^{u}=s_{L i}^{u} e^{i \delta_{L i}^{u}}}
\end{aligned}
$$

where $\tilde{s}_{L i}^{u}$ are complex sines of angles in the 14,24,34 family planes parameterizing the mixing of the first three families with the vector-like quark, as in (4.11), (4.12), and we can choose $\delta_{L 1}^{u}=0$ without loss of generality.

Since the matrix of Yukawa couplings and the mass matrix are not proportional, also the couplings of quarks with the real Higgs are not diagonal. In particular, left-handed SM quarks are coupled with $t_{R}^{\prime}$ with coupling constants which can be in principle of order $O(1)$, as shown in eq. (4.21) (with the replacements $\left.h_{d i} \rightarrow h_{u i}, d, s, b \rightarrow u, c, t\right)$.

Let us also notice that, because of the large mixing with the first family, the extra quark $t^{\prime}$ would mainly decay into $u$ or $d$ quark via the couplings with $W, Z, H$. The CMS 
experiment put a lower limit $M_{t^{\prime}} \gtrsim 685 \mathrm{GeV}$ [82], which implies that $\left|V_{t^{\prime} d}\right| \simeq 0.03$ can be obtained if $\left|h_{u 1}\right| \gtrsim 0.1$, much larger than the Yukawa constant of the bottom quark. In turn, by taking $\left|V_{L 4 u}\right|>0.03$ in $M_{t^{\prime}}=\left|h_{u 1}\right| v_{w} /\left|V_{t^{\prime} d}\right|$, and assuming (for the perturbativity) $h_{u 1} \lesssim 1$, there is an upper limit on the extra quark mass, $M_{t^{\prime}} \lesssim 6 \mathrm{TeV}$.

In the following sections experimental limits from FCNC and electroweak observables are examined. The results are summarized in section 5.4, in table 7 and figures 14, 16 .

\subsection{Limits from rare $D$ mesons decays}

D-mesons rare decays can limit mixings of the standard quarks with the additional vectorlike singlet up-quark $t^{\prime}$. In fact, the non-diagonal couplings of $Z$-boson with light quarks in eqs. (5.7) induce at tree level flavour changing leptonic and semileptonic decays of D-mesons:

$$
\mathcal{L}_{\text {new }}=\frac{4 G_{F}}{\sqrt{2}} V_{L 4 u}^{*} V_{L 4 c}\left(\overline{u_{L}} \gamma^{\mu} c_{L}\right)\left[\left(-\frac{1}{2}+\sin ^{2} \theta_{W}\right)\left(\overline{\ell_{L}} \gamma_{\mu} \ell_{L}\right)+\sin ^{2} \theta_{W}\left(\overline{\ell_{R}} \gamma_{\mu} \ell_{R}\right)\right]+\text { h.c. }
$$

The most stringent constraint from semileptonic decays comes from the experimental limit [20]:

$$
\operatorname{Br}\left(D^{+} \rightarrow \pi^{+} \mu^{+} \mu^{-}\right) \exp <7.3 \cdot 10^{-8} \quad 90 \% \text { C.L. }
$$

Neglecting the SM contribution, we can impose the experimental limit on the new contribution:

$$
\begin{aligned}
\operatorname{Br}\left(D^{+} \rightarrow \pi^{+} \mu^{+} \mu^{-}\right)_{\text {new }} & \simeq \operatorname{Br}\left(D^{+} \rightarrow \pi^{0} \mu^{+} \nu_{\mu}\right) \frac{2\left|V_{L 4 u}^{*} V_{L 4 c}\right|^{2}\left[\left(-\frac{1}{2}+\sin ^{2} \theta_{W}\right)^{2}+\sin ^{4} \theta_{W}\right]}{\left|V_{c d}\right|^{2}} \\
& <7.3 \cdot 10^{-8}
\end{aligned}
$$

from which

$$
\left|V_{L 4 u}^{*} V_{L 4 c}\right|<2.0 \cdot 10^{-3}\left[\frac{\mathrm{Br}_{\max }}{7.3 \cdot 10^{-8}}\right]^{\frac{1}{2}}
$$

where we take advantage of the experimental branching ratio $\operatorname{Br}\left(D^{+} \rightarrow \pi^{0} \mu^{+} \nu_{\mu}\right)=(3.50 \pm$ $0.15) \cdot 10^{-3}[20] . \mathrm{Br}_{\max }$ is defined as in eq. (4.123) as the maximum allowed branching ratio induced by the beyond SM amplitude alone.

The experimental constraint on the leptonic decay $D^{0} \rightarrow \mu^{+} \mu^{-}$is [20]:

$$
\operatorname{Br}\left(D^{0} \rightarrow \mu^{+} \mu^{-}\right)_{\exp }<6.2 \cdot 10^{-9} \quad 90 \% \text { C.L. }
$$

Neglecting the SM short- and long- distance contribution, the limit on the new contribution:

$$
\operatorname{Br}\left(D^{0} \rightarrow \mu^{+} \mu^{-}\right)_{\text {new }}=\operatorname{Br}\left(D^{+} \rightarrow \mu^{+} \nu_{\mu}\right) \frac{1}{2} \frac{\tau\left(D^{0}\right) M_{D^{0}} \sqrt{1-4 \frac{m_{\mu}^{2}}{M_{D^{0}}^{2}}}}{\tau\left(D^{+}\right) M_{D^{+}}\left(1-\frac{m_{\mu}^{2}}{M_{D^{+}}^{2}}\right)^{2}} \frac{\left|V_{L 4 u}^{*} V_{L 4 c}\right|^{2}}{\left|V_{c d}\right|^{2}}<6.2 \cdot 10^{-9}
$$

It is obtained that:

$$
\left|V_{L 4 u}^{*} V_{L 4 c}\right|<2.0 \cdot 10^{-3}\left[\frac{\mathrm{Br}_{\max }}{6.2 \cdot 10^{-9}}\right]^{\frac{1}{2}}
$$

using the values in table 4 , with $\operatorname{Br}\left(D^{+} \rightarrow \mu^{+} \nu_{\mu}\right)=(3.74 \pm 0.17) \cdot 10^{-4}$. 


\subsection{Limits from neutral mesons systems}

\subsection{1 $\quad D^{0}-\bar{D}^{0}$ mixing}

As described in section 4.2.3, the experimental result (4.101) for the mass difference in the $D^{0}$ system allows values which can be two orders of magnitude higher than the SM shortdistance expectation, while long-distance effects cannot be computed reliably. Then, new physics scenarios can in principle account for the experimental mass difference $\Delta m_{D \text { exp }}$ in eq. (4.101).

The non-diagonal couplings of Higgs and $Z$ bosons with quarks bring additional contributions to the transition $\bar{D}^{0} \leftrightarrow D^{0}$, both at tree level and loop level. Analogously to eq. (4.79), the corresponding effective Lagrangian reads:

$$
\begin{aligned}
\mathcal{L}_{\Delta C=2}^{\text {new }} & \approx-\frac{G_{F}}{\sqrt{2}}\left(V_{L 4 u}^{*} V_{L 4 c}\right)^{2}\left(1+\frac{G_{F} M_{t^{\prime}}^{2}}{8 \sqrt{2} \pi^{2}}\right)\left(\overline{u_{L}} \gamma^{\mu} c_{L}\right)^{2}+\text { h.c. }= \\
& =-\frac{G_{F}}{\sqrt{2}}\left(V_{L 4 u}^{*} V_{L 4 c}\right)^{2} f\left(M_{t^{\prime}}\right)\left(\overline{u_{L}} \gamma^{\mu} c_{L}\right)^{2}+\text { h.c. } \\
f\left(M_{t^{\prime}}\right) & =1+\left(\frac{M_{t^{\prime}}}{3.1 \mathrm{TeV}}\right)^{2}
\end{aligned}
$$

Then, the new operator (5.17) can generate the mass difference:

$$
\Delta m_{D \text { new }} \approx 2\left|M_{12 \text { new }}^{D}\right| \simeq \frac{2}{3} \frac{G_{F}}{\sqrt{2}} f\left(M_{t^{\prime}}\right)\left|V_{L 4 u}^{*} V_{L 4 c}\right|^{2} f_{D}^{2} m_{D^{0}}
$$

We can think that $\Delta m_{D \text { new }}$ is the dominant contribution to the mass difference. Then, as in section 4.2.3, we can set an indicative limit by using the experimental result (4.101) within two error bars, $\Delta m_{D \text { new }}<8.2 \times 10^{-15} \mathrm{GeV}$, as in (4.104):

$$
\left|V_{L 4 u}^{*} V_{L 4 c}\right|<1.3 \times 10^{-4}\left[\frac{f(1 \mathrm{TeV})}{f\left(M_{t^{\prime}}\right)}\right]^{1 / 2}\left[\frac{\Delta m_{D \max }}{8.2 \cdot 10^{-15}}\right]
$$

\subsection{2 $\quad K^{0}-\bar{K}^{0}$ mixing}

In the SM, the short-distance contribution to the transition $K^{0}(d \bar{s}) \leftrightarrow \bar{K}^{0}(\bar{d} s)$ arises from weak box diagrams (figure 8), which corresponds to the effective Lagrangian in eq. (4.64).

In the scenario with extra up-type quark, the mixing of the SM quarks with the $t^{\prime}$ quark in the charged current in eq. (5.2) gives additional contributions to the $K^{0}$ mixing, in interference with the SM. In fact, the same operator as in (4.64) is originated by box diagrams with internal $t^{\prime}$-quark running in the loop, as shown in figure 13:

$$
\begin{aligned}
\mathcal{L}_{\Delta S=2}^{\text {new }}=-\frac{G_{F}^{2} m_{W}^{2}}{4 \pi^{2}}( & \left(V_{t^{\prime} s}^{*} V_{t^{\prime} d}\right)^{2} S\left(x_{t^{\prime}}\right)+2\left(V_{t^{\prime} s}^{*} V_{t^{\prime} d}\right)\left(V_{c s}^{*} V_{c d}\right) S\left(x_{c}, x_{t^{\prime}}\right)+ \\
& \left.+2\left(V_{t^{\prime} s}^{*} V_{t^{\prime} d}\right)\left(V_{t s}^{*} V_{t d}\right) S\left(x_{t}, x_{t^{\prime}}\right)\right)\left(\overline{s_{L}} \gamma^{\mu} d_{L}\right)\left(\overline{s_{L}} \gamma^{\mu} d_{L}\right)+\text { h.c. }
\end{aligned}
$$

where $x_{a}=\frac{m_{a}^{2}}{m_{W}^{2}}$ and $S_{0}(x, y)$ are the Inami-Lim functions [93] in eqs. (4.65), (4.66).

We constrain the modulus and imaginary part of the BSM contribution (5.21) to the mixing mass following section 4.2.1. The mixing mass in the SM $M_{12}^{\mathrm{SM}}$ is given in eq. (4.68). 


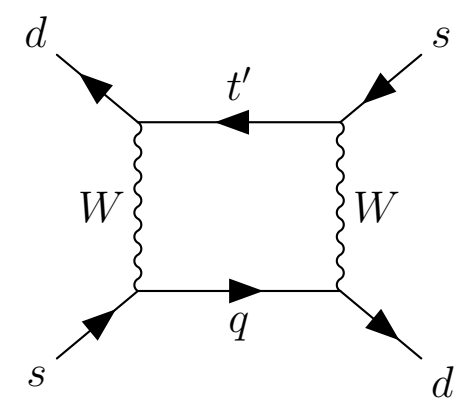

Figure 13. New contribution to $\bar{K}^{0} \rightarrow K^{0}$ mixing, $q=u, c, t, t^{\prime}$.

Regarding the CP-conserving part, in order to estimate the constraint on the new mixing elements we can impose:

$$
\left|M_{12}^{\text {new }}\right|<\left|M_{12}^{\mathrm{SM}}\right| \Delta_{K}
$$

where $\Delta_{K}$ is real and positive. As an indicative estimate, we will evaluate for $\Delta_{K}=1$, as in section 4.2.1. This is analogous to comparing the coefficients of the effective operators in eqs. (4.64) and (5.21). In this case the upper bound is basically determined by constraining the contribution of the box diagram with $t^{\prime}$ running in the loop:

$$
\begin{aligned}
\left|V_{t^{\prime} s}^{*} V_{t^{\prime} d}\right|^{2} S_{0}\left(x_{t^{\prime}}\right) & <S_{0}\left(x_{c}\right)\left(V_{c s}^{*} V_{c d}\right)^{2} \Delta_{K} \\
\left|V_{t^{\prime} s}^{*} V_{t^{\prime} d}\right| & <5.2 \times 10^{-4}\left[\frac{1 \mathrm{TeV}}{M_{t^{\prime}}}\right]\left[\Delta_{K}\right]^{1 / 2}
\end{aligned}
$$

The scaling with $t^{\prime}$ mass in eq. (5.23) holds since $S_{0}\left(x_{t^{\prime}}\right) \sim \frac{1}{4} x_{t^{\prime}}$ for $M_{t^{\prime}} \gtrsim 2 \mathrm{TeV}$.

Regarding the contribution to the CP-violating parameter $\epsilon_{K}$, we can estimate the upper bound on the new operator by constraining the imaginary part of the new contribution to the mixing mass $\left|\operatorname{Im} M_{12}^{\text {new }}\right|$ to be a fraction $\Delta_{\epsilon_{K}}$ of the SM contribution, as in eq. (4.85):

$$
\left|\operatorname{Im} M_{12}^{\text {new }}\right|<\left|\operatorname{Im} M_{12}^{\mathrm{SM}}\right| \Delta_{\epsilon_{K}}
$$

At leading order of the new physics contribution, eq. (5.24) is equivalent to comparing the magnitude of the imaginary part of operators (4.64) and (5.21), in the standard parameterization of CKM matrix:

$$
\begin{aligned}
& \mid \operatorname{Im}\left(\left(V_{t^{\prime} s}^{*} V_{t^{\prime} d}\right)^{2} S\left(x_{t^{\prime}}\right)+2\left(V_{t^{\prime} s}^{*} V_{t^{\prime} d}\right)\left(V_{c s}^{*} V_{c d}\right) S_{0}\left(x_{c}, x_{t^{\prime}}\right)+\right. \\
& \left.+2\left(V_{t^{\prime} s}^{*} V_{t^{\prime} d}\right)\left(V_{t s}^{*} V_{t d}\right) S_{0}\left(x_{t}, x_{t^{\prime}}\right)\right)|<| \operatorname{Im}\left(\mathcal{C}_{\mathrm{SM}}\right) \mid \Delta_{\epsilon_{K}}
\end{aligned}
$$

with

$$
\mathcal{C}_{\mathrm{SM}}=\eta_{1}\left(V_{c s}^{*} V_{c d}\right)^{2} S_{0}\left(x_{c}\right)+\eta_{2}\left(V_{t s}^{*} V_{t d}\right)^{2} S_{0}\left(x_{t}\right)+2 \eta_{3}\left(V_{c s}^{*} V_{c d}\right)\left(V_{t s}^{*} V_{t d}\right) S_{0}\left(x_{c}, x_{t}\right)
$$

The constraint depends on the mass of the extra quark, since the first term of eq. (5.21) scales as $S_{0}\left(x_{t^{\prime}}\right) \sim \frac{1}{4} M_{t^{\prime}}^{2} / m_{W}^{2}$ for $M_{t^{\prime}} \gtrsim 2 \mathrm{TeV}$, while the second and third components are logarithmically growing with $t^{\prime}$ mass. We make an estimation choosing $\Delta_{\epsilon_{K}}=0.4$, 


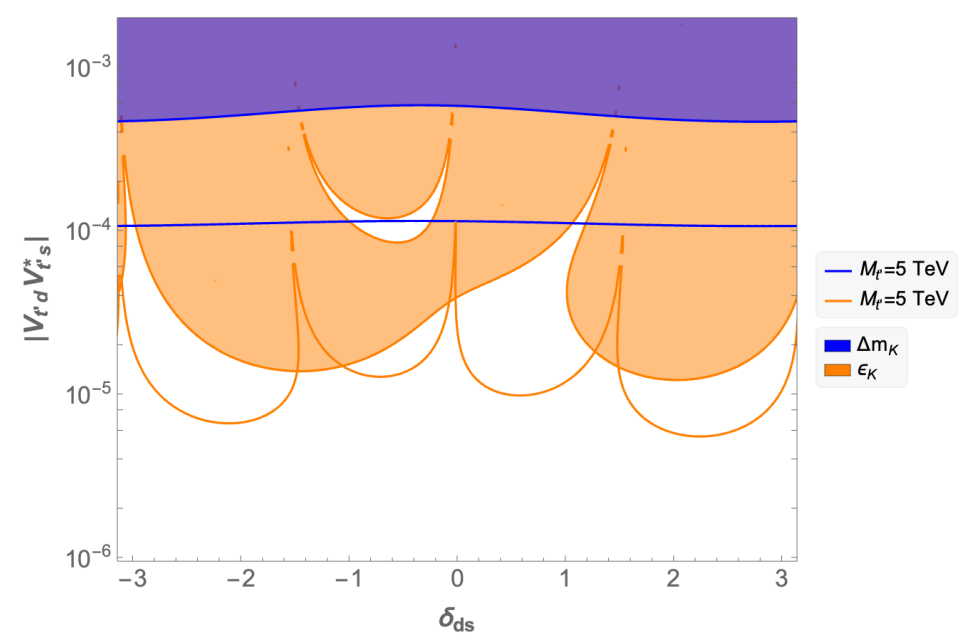

Figure 14. Upper limits on the product $\left|V_{t^{\prime} s}^{*} V_{t^{\prime} d}\right|$ from neutral kaon mixing, obtained from the constraint in eqs. (5.22), (5.24) with $\Delta_{K}=1, \Delta_{\epsilon_{K}}=0.4$, as a function of the relative phase $\delta_{d s}=\arg \left(V_{t^{\prime} s}^{*} V_{t^{\prime} d}\right)$. The blue and orange lines indicate where the upper limit shifts for $M_{t^{\prime}}=5 \mathrm{TeV}$.

corresponding to an equivalent scale of the new operator $\Lambda_{d s, \operatorname{Im}}=2.5 \cdot 10^{4} \mathrm{TeV}$, as defined in eq. (4.87). The upper bounds on the product $V_{t^{\prime} s}^{*} V_{t^{\prime} d}$ obtained with $\Delta_{K}=1, \Delta_{\epsilon_{K}}=0.4$ are shown in figure 14, where $\delta_{d s}=\arg \left(V_{t^{\prime} s}^{*} V_{t^{\prime} d}\right)$. The excluded region is shown for $M_{t^{\prime}}=1 \mathrm{TeV}$, while the lines show the shift of the upper limit for $M_{t^{\prime}}=5 \mathrm{TeV}$.

\subsection{3 $B_{s, d^{0}}^{0} \bar{B}_{s, d}^{0}$ mixing}

As illustrated in section 4.2 .2 , in the SM the dominant contribution to $B_{d}^{0}(\bar{b} d)-\bar{B}_{d}^{0}(\bar{d} b)$ and $B_{s}^{0}(\bar{b} s)-\bar{B}_{s}^{0}(\bar{s} b)$ mixings comes from box-diagrams with internal top-quark, with effective Lagrangian in eq. (4.90).

In the scenario with extra up-type quark, the mixing of the SM quarks with the $t^{\prime}$-quark in the charged current in eq. (5.2) contribute to neutral B-mesons mixing, in interference with the SM. In fact, the same operator as in (4.90) is originated by box diagrams with internal $t^{\prime}$ and $t, t^{\prime}$-quarks:

$$
\begin{aligned}
& \mathcal{L}_{\Delta B(d)=2}^{\text {new }}=-\frac{G_{F}^{2} m_{W}^{2}}{4 \pi^{2}}\left(\left(V_{t^{\prime} b}^{*} V_{t^{\prime} d}\right)^{2} S_{0}\left(x_{t^{\prime}}\right)+2\left(V_{t^{\prime} b}^{*} V_{t^{\prime} d}\right)\left(V_{t b}^{*} V_{t d}\right) S_{0}\left(x_{t}, x_{t^{\prime}}\right)\right)\left(\overline{b_{L}} \gamma^{\mu} d_{L}\right)^{2}+\text { h.c. } \\
& \mathcal{L}_{\Delta B(s)=2}^{\text {new }}=-\frac{G_{F}^{2} m_{W}^{2}}{4 \pi^{2}}\left(\left(V_{t^{\prime} b}^{*} V_{t^{\prime} s}\right)^{2} S_{0}\left(x_{t^{\prime}}\right)+2\left(V_{t^{\prime} b}^{*} V_{t^{\prime} s}\right)\left(V_{t b}^{*} V_{t s}\right) S_{0}\left(x_{t}, x_{t^{\prime}}\right)\right)\left(\overline{b_{L}} \gamma^{\mu} s_{L}\right)^{2}+\text { h.c. }
\end{aligned}
$$

where $x_{a}=\frac{m_{a}^{2}}{m_{W}^{2}}$ and $S\left(x_{a}\right)$ are the Inami-Lim functions [93] given in eqs. (4.65), (4.66). The c-quark contribution is negligible.

We can constrain the new contribution to be less than a fraction $\Delta_{B_{(d, s)}}$ of the SM contribution $\Delta M_{d, s}^{\mathrm{SM}}=2\left|M_{12}^{(d, s) \mathrm{SM}}\right|$ given in eq. (4.93), as:

$$
\left|M_{12}^{(d, s) \text { new }}\right|<\left|M_{12}^{(d, s) \mathrm{SM}}\right| \Delta_{B_{d, s}}
$$



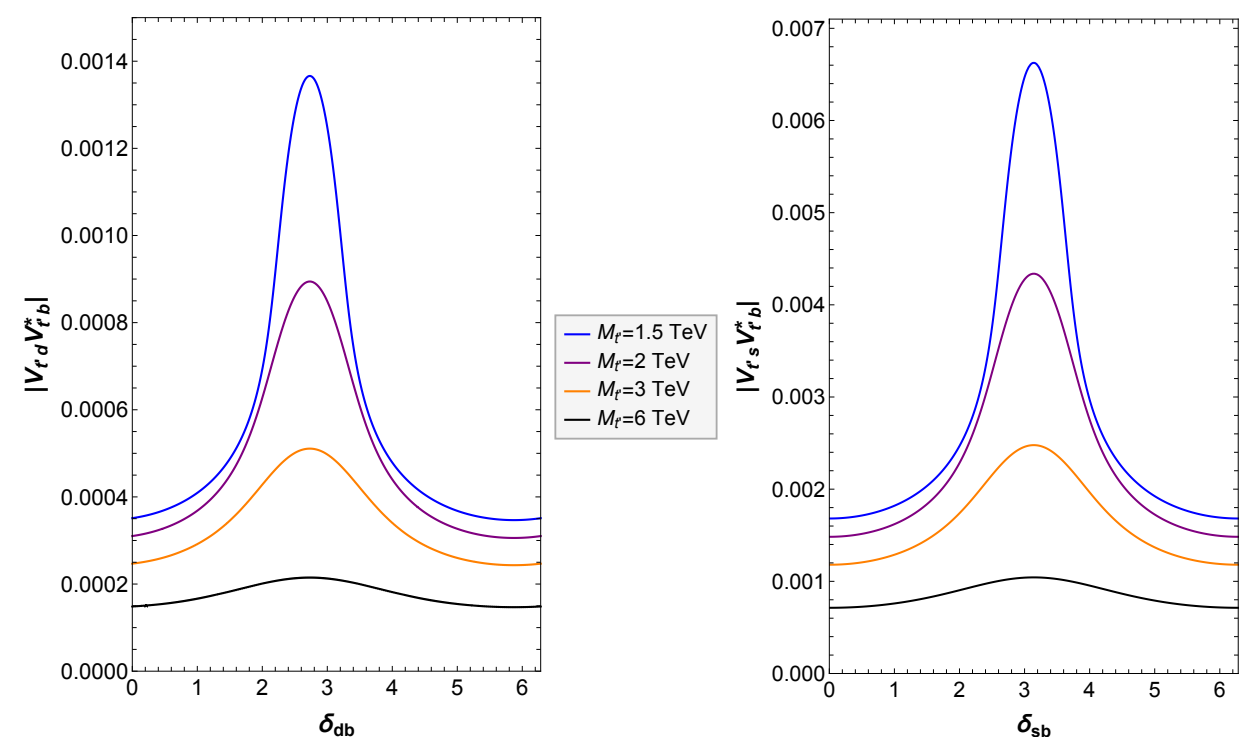

Figure 15. Upper limits on the products of the mixing elements $\left|V_{t^{\prime} s}^{*} V_{t^{\prime} d}\right|,\left|V_{t^{\prime} s}^{*} V_{t^{\prime} d}\right|$ from neutral B-mesons mixing, obtained from the constraint in eqs. eqs. (5.30) with $\Delta_{B_{d, s}}=0.3$, as a function of the relative phases $\delta_{d b}=\arg \left(V_{t^{\prime} b}^{*} V_{t^{\prime} d}\right), \delta_{s b}=\arg \left(V_{t^{\prime} b}^{*} V_{t^{\prime} s}\right)$, evaluated for the values of extra quark masses $M_{t^{\prime}}=1.5,2,3,6 \mathrm{TeV}$ (blue, purple, orange and black curves respectively).

This is analogous to comparing the coefficients of the effective operators in eqs. (5.27), (5.28) with the SM ones (4.90):

$$
\left|\left(V_{t^{\prime} b}^{*} V_{t^{\prime} d / s}\right)^{2} S_{0}\left(x_{t^{\prime}}\right)+2\left(V_{t^{\prime} b}^{*} V_{t^{\prime} d / s}\right)\left(V_{t b}^{*} V_{t d / s}\right) S_{0}\left(x_{t}, x_{t^{\prime}}\right)\right|<S\left(x_{t}\right)\left|V_{t b}^{*} V_{t d / s}\right|^{2} \Delta_{B_{d, s}}
$$

where we evaluate the constraint at leading order of both SM and new physics contributions. As in section 4.2 .2 , we use $\Delta_{B_{(d, s)}}=0.3$ as a benchmark value. Then, by taking $M_{t^{\prime}}=1 \mathrm{TeV}$, we obtain:

$$
\begin{aligned}
& \left|V_{t^{\prime} b}^{*} V_{t^{\prime} d}\right|<0.4 \div 2.6 \times 10^{-3} \\
& \left|V_{t^{\prime} b}^{*} V_{t^{\prime} s}\right|<0.2 \div 1.2 \times 10^{-2}
\end{aligned}
$$

depending on the relative phases $\delta_{d b}=\arg \left(V_{t^{\prime} b}^{*} V_{t^{\prime} d}\right), \delta_{s b}=\arg \left(V_{t^{\prime} b}^{*} V_{t^{\prime} s}\right)$. The constraints become more stringent for higher values of $t^{\prime}$ mass. The contribution from the mixed box diagrams with $t, t^{\prime}$ grows logarithmically with $M_{t^{\prime}}$, but the contribution from the boxdiagram with $t^{\prime}$ increases linearly with the mass for $M_{t^{\prime}} \gtrsim 2 \mathrm{TeV}$. Then, for $M_{t^{\prime}} \gtrsim 3 \mathrm{TeV}$ the upper limits start to decrease as $M_{t^{\prime}}^{-1}$. The upper bound on the mixing elements $\left|V_{t^{\prime} b}^{*} V_{t^{\prime} d}\right|,\left|V_{t^{\prime} b}^{*} V_{t^{\prime} d}\right|$ as a function of the relative phase of the elements is shown in figure 15 for increasing values of the extra quark $t^{\prime}$ mass.

\subsection{Limits from $Z$-boson physics}

The presence of vector-like quarks affect both off-diagonal couplings and diagonal couplings of Z-boson with quarks, changing the prediction of the observables related to the $Z$ boson physics. In this BSM scenario, the partial decay rate into hadrons $\Gamma(Z \rightarrow$ had) (which also 
corresponds to the deviation of the total $Z$ decay rate $\Gamma(Z)$, since there are not additional leptons) would be changed with respect to the SM expectation by:

$$
\begin{aligned}
& \Gamma(\mathrm{had})-\Gamma(\mathrm{had})_{\mathrm{SM}}=\Gamma(Z)-\Gamma(Z)_{\mathrm{SM}}= \\
& =\frac{G_{F} M_{Z}^{3}}{\sqrt{2} \pi}\left[\sum_{i, j=u, c}\left|\frac{1}{2} \sum_{k=1}^{3} V_{L k i}^{*} V_{L k j}-\frac{2}{3} \sin ^{2} \theta_{W} \delta_{i j}\right|^{2}-2\left(\frac{1}{2}-\frac{2}{3} \sin ^{2} \theta_{W}\right)^{2}\right]= \\
& =\frac{G_{F} M_{Z}^{3}}{\sqrt{2} \pi}\left[\sum_{q=u, c}\left(\frac{1}{2}\left(1-\left|V_{L 4 q}\right|^{2}\right)-\frac{2}{3} \sin ^{2} \theta_{W}\right)^{2}+\frac{1}{2}\left|V_{L 4 u}^{*} V_{L 4 c}\right|^{2}-2\left(\frac{1}{2}-\frac{2}{3} \sin ^{2} \theta_{W}\right)^{2}\right] \approx \\
& \approx \frac{G_{F} M_{Z}^{3}}{\sqrt{2} \pi}\left(-\frac{1}{2}+\frac{2}{3} \sin ^{2} \theta_{W}\right)\left(\left|V_{L 4 u}\right|^{2}+\left|V_{L 4 c}\right|^{2}\right)<0
\end{aligned}
$$

where QED and QCD corrections factor $\approx 1.050$ should be included. As shown in eq. (5.33), the prediction for the decay rate is lowered with respect to the SM one. Since the SM expectation (4.141) is below the experimental result (4.142), following section 4.4, we can choose to use the limit value of the SM prediction $\Gamma(Z \rightarrow \text { had })_{\mathrm{SM}}=1.7419 \mathrm{GeV}$. Then we can impose that $\Gamma(Z \rightarrow$ had $)$ should stay in the $95 \%$ C.L. interval of the experimental value $\Gamma(Z \rightarrow \text { had })_{\exp }$, which means:

$$
\left|V_{L 4 u}\right|^{2}+\left|V_{L 4 c}\right|^{2}<2.0 \cdot 10^{-3}\left[\frac{\Delta \Gamma_{Z}}{1.4 \times 10^{-3} \mathrm{GeV}}\right]
$$

where $\Delta \Gamma_{Z}$ is defined by $\Gamma(Z \rightarrow$ had $)-\Gamma(Z \rightarrow \text { had })_{\mathrm{SM}}<\Delta \Gamma_{Z}$ as in eq. (4.143). The constraint is satisfied if both $\left|V_{L 4 u, c}\right| \lesssim 0.032$. With $V_{L 4 c}=0$, the constraint implies:

$$
\left|V_{L 4 u}\right|^{2}<2.0 \cdot 10^{-3}, \quad\left|V_{L 4 u}\right|<0.044
$$

which is extremely close to the value needed to solve the CKM unitarity problem (for example, at $95 \%$ C.L. $\left|V_{L 4 u}\right|=0.043_{-0.015}^{+0.011}$ (4.8) using our conservative average for $V_{u d}(2.10)$ ).

The presence of the additional up-type isosinglet changes the predictions for the asymmetries as in eq. (4.146). However, the expected value of $A_{c}$ remains in the $95 \%$ C.L. interval of the experimental determination $A_{c}=0.670 \pm 0.027$ with $V_{L 4 c}<0.24$, and it is within one errorbar of the $A_{c}$ value extracted from $A_{F B}^{(0, c)}$ with $V_{L 4 c}<0.28$.

\subsection{Summary of experimental limits}

As illustrated in section 2, the analysis of the latest determinations of $V_{u s}$ obtained from kaon decays and of $V_{u d}$ from beta decays results in a deviation from unitarity of the first row of the CKM matrix. The SM unitarity relation (1.3) can be modified into the relation in eq. (5.5) if an extra vector-like up-type quark $u_{L 4}, u_{R 4}$ participates in the mixing of the SM families. A quite large mixing with the first family, $\left|V_{t^{\prime} d}\right| \approx\left|V_{L 4 u}\right| \approx 0.043(7)$ (5.6), is needed in order to explain the data, see eq. (5.6) (see also table 2, where in this scenario $\left.\delta_{\mathrm{CKM}}=\left|V_{L 4 u}\right|^{2}\right)$. In this way, the values of $V_{u s}$ obtained from the three determinations can be shifted as shown in figure 5 , where $\left|V_{t^{\prime} d}\right| \approx\left|V_{L 4 u}\right|$ plays the same role as $\left|V_{u b^{\prime}}\right|$. Then, we need to verify if such large mixing is compatible with experimental constraints from flavour changing decays and electroweak observables. 
In table 7 constraints obtained in this section on the mixing of the new up-type vectorlike quark with the SM families are summarized $\left(\left|V_{t^{\prime} d}\right| \approx\left|V_{L 4 u}\right|=0.03\right.$ is used as a conservative benchmark value).

In figure 16 the allowed area in the parameter space of $\left|V_{t^{\prime} s}\right|$ and $\left|V_{L 4 c}\right|$ is shown, using $\left|V_{t^{\prime} d}\right|=0.04$ and $\left|V_{t^{\prime} d}\right|=0.03$ as benchmark values.

The blue area is excluded by constraints on neutral K-mesons system, as obtained in section 5.2.2 and summarized in figure 14. Since the constraint on the CP violating contribution can be avoided for specific values of the phase $\delta_{d s}=\arg \left(V_{t^{\prime} s}^{*} V_{t^{\prime} d}\right)$, in figure 16 we conservatively use the bound on the contribution to the mass difference $\Delta m_{K}(5.23)$ for $M_{t^{\prime}}=1 \mathrm{TeV}$, which gives:

$$
\left|V_{t^{\prime} s}^{*} V_{t^{\prime} d}\right|<5.2 \times 10^{-4}\left[\frac{1 \mathrm{TeV}}{M_{t^{\prime}}}\right], \quad\left|V_{t^{\prime} s}\right|<1.7 \cdot 10^{-2}\left[\frac{0.03}{\left|V_{L 4 u}\right|}\right]\left[\frac{1 \mathrm{TeV}}{M_{t^{\prime}}}\right]
$$

The red area is excluded by the constraints on $\left|V_{L 4 c}\right|$ from $D^{0}$ systems, for $M_{t^{\prime}}=1 \mathrm{TeV}$, as reported in eq. (5.20):

$$
\left|V_{L 4 u}^{*} V_{L 4 c}\right|<1.3 \times 10^{-4}\left[\frac{f(1 \mathrm{TeV})}{f\left(M_{t^{\prime}}\right)}\right]^{1 / 2}, \quad\left|V_{L 4 c}\right|<4.2 \cdot 10^{-3}\left[\frac{0.03}{\left|V_{L 4 u}\right|}\right]
$$

where $f\left(M_{t^{\prime}}\right)$ is defined in eq. (5.18). As discussed in section 5.2.1, the constraint is obtained by allowing the new contribution to the mass difference in $D^{0}$ mesons system to account for the experimental value $\Delta m_{D \text { exp }}$, in the $2 \sigma$ interval. Then, also in this case, knowledge of the long distance contribution (and corresponding sign) can exclude this possibility if it will saturate the experimental value or, viceversa, the extra singlet quark can be thought as a way to explain the value of the mass difference in neutral D-mesons system in case of deviation between experimental and expected value. We can also notice that, taking into account the relations (5.9) $V_{L 4 u} \approx-h_{u 1}^{*} v_{w} / M_{t^{\prime}}, V_{L 4 c} \approx-h_{u 2}^{*} v_{w} / M_{t^{\prime}}$, where $h_{d i}$ are the Yukawa couplings defined in eq. (5.1), from eq. (5.37) follows that the coupling of the 4th species with the 2 nd family $h_{d 2}$ should be one order of magnitude smaller than the coupling with the first one $h_{d 1}$.

From eq. (5.8) we have:

$$
\begin{aligned}
\left|V_{t^{\prime} s}\right| & \approx\left|V_{t^{\prime} d} V_{u s}-V_{L 4 c} V_{c s}+V_{t^{\prime} b} V_{t s}\right| \approx \\
& \approx|| V_{t^{\prime} d}\left|V_{u s}+e^{i \delta_{L 21}^{u}}\right| V_{L 4 c}\left|V_{c s}-e^{-i \delta_{d b}}\right| V_{t^{\prime} b}|| V_{t s}||
\end{aligned}
$$

where we used the parameterization in eqs. (5.9), and $\arg \left(V_{t^{\prime} d}\right) \approx \delta_{L 1}^{u},-\delta_{d b}=\arg \left(V_{t^{\prime} b} V_{t^{\prime} d}^{*}\right)$ $\approx \delta_{L 31}^{u}=\delta_{L 3}^{u}-\delta_{L 1}^{u}$, and we can choose $\delta_{L 1}^{u}=0$ without loss of generality. The green region is excluded by the relation (5.38) for any value of the relative phases, using the values for $V_{t^{\prime} b}$ allowed by the limit from the B-mesons system in eq. (5.31), figure 15:

$$
\left|V_{t^{\prime} b}^{*} V_{t^{\prime} d}\right|<2.6 \times 10^{-3}, \quad\left|V_{t^{\prime} b}\right|<8.5 \times 10^{-2}\left[\frac{0.03}{\left|V_{t^{\prime} d}\right|}\right]
$$

The conditions on $V_{t^{\prime} s}$ and $V_{t^{\prime} b}$ also satisfy the constraint on the product $V_{t^{\prime} b}^{*} V_{t^{\prime} s}(5.32)$ for the relative phase needed in order to have the upper bounds (5.36), (5.39), $\delta_{s b}=$ $\operatorname{Arg}\left(V_{t^{\prime} b}^{*} V_{t^{\prime} s}\right)=\delta_{d b}-\delta_{d s} \approx 2.77$. 


\begin{tabular}{|lll|}
\hline Process & Constraint & \\
\hline$D^{+} \rightarrow \pi^{+} \mu^{+} \mu^{-}$ & $\left|V_{L 4 c} V_{L 4 u}^{*}\right|<2.0 \times 10^{-3}$ & $\left|V_{L 4 c}\right|<6.7 \times 10^{-2}\left[\frac{0.03}{\left.\mid V_{L 4 u}\right]}\right]$ \\
$D^{0} \rightarrow \pi^{0} e^{+} e^{-}$ & $\left|V_{L 4 c} V_{L 4 u}^{*}\right|<3.3 \times 10^{-2}$ & \\
$D^{0} \rightarrow \mu^{+} \mu^{-}$ & $\left|V_{L 4 c} V_{L 4 u}^{*}\right|<2.0 \times 10^{-3}$ & $\left|V_{L 4 c}\right|<6.8 \times 10^{-2}\left[\frac{0.03}{\mid V_{L 4 u}}\right]$ \\
$D^{0}-\bar{D}^{0}$ & $\left|V_{L 4 c} V_{L 4 u}^{*}\right|<1.3 \times 10^{-4}$ & $\left|V_{L 4 c}\right|<4.2 \times 10^{-3}\left[\frac{0.03}{\mid V_{L 4 u}}\right]$ \\
\hline$K^{0}-\bar{K}^{0}$ & $\left|V_{t^{\prime} s}^{*} V_{t^{\prime} d}\right|<(0.1 \div 5.8) \times 10^{-4}$ & $\left|V_{t^{\prime} s}\right|<1.9 \times 10^{-2}\left[\frac{0.03}{\left|V_{L 4 u}\right|}\right]$ \\
\hline$B^{0}-\bar{B}^{0}$ & $\left|V_{t^{\prime} b}^{*} V_{t^{\prime} d}\right|<(0.4 \div 2.6) \times 10^{-3}$ & $\left|V_{t^{\prime} b}\right|<8.5 \times 10^{-2}\left[\frac{0.03}{\left|V_{L 4 u}\right|}\right]$ \\
\hline$B_{s}^{0}-\bar{B}_{s}^{0}$ & $\left|V_{t^{\prime} b}^{*} V_{t^{\prime} s}\right|<(0.2 \div 1.2) \times 10^{-2}$ & \\
\hline$\Gamma_{Z}, \Gamma \rightarrow \operatorname{hadr}$ & $\left|V_{L 4 u}\right|^{2}+\left|V_{L 4 c}\right|^{2}<2.0 \cdot 10^{-3},\left|V_{L 4 u}\right|<0.044$ & \\
\hline
\end{tabular}

Table 7. Limits on the mixing of the SM three families with a vector-like up-type isosinglet $t^{\prime}$. The upper bounds obtained from neutral mesons mixing depend on the relative phases of the mixing elements and on the extra quark mass. Here the limits are computed for $M_{t^{\prime}}=1 \mathrm{TeV}$ (see the text for details). Regarding the elements of CKM matrix, $V_{t^{\prime} d} \approx-V_{L 4 u}, V_{t^{\prime} s} \approx-V_{L 4 u} V_{u s}-V_{L 4 c} V_{c s}-V_{L 4 t} V_{t s}$ and $V_{t^{\prime} b} \approx-V_{L 4 t}$, as in eq. (4.18).
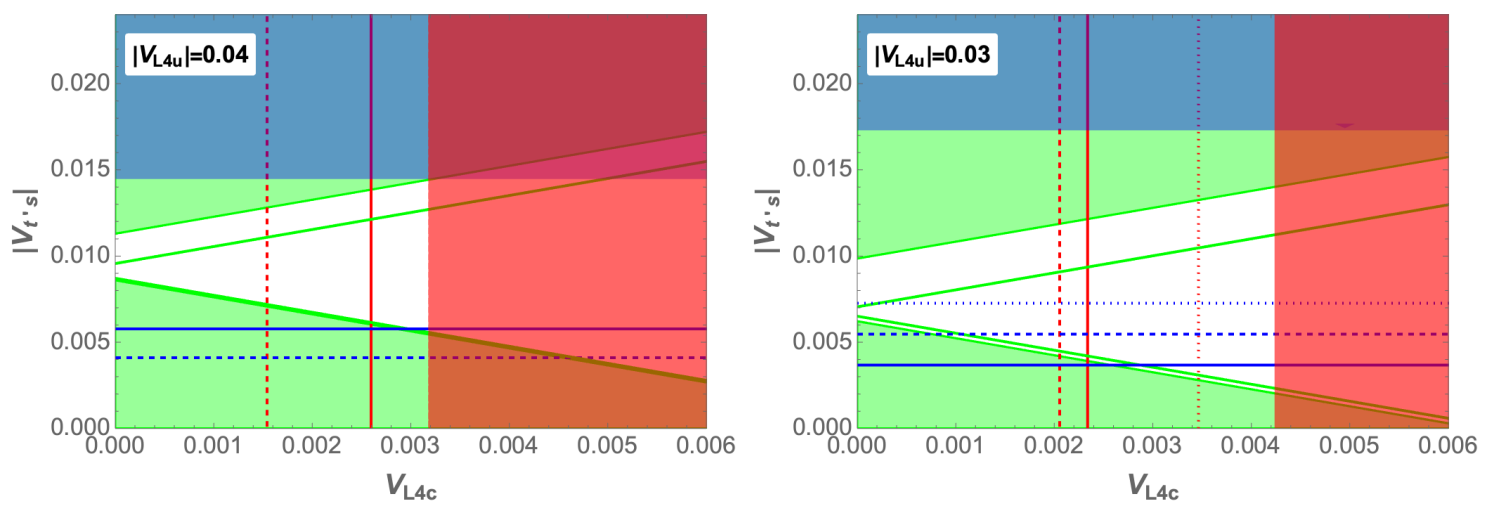

Figure 16. Constraints on $\left|V_{t^{\prime} s}\right|$ and $\left|V_{L 4 c}\right|$. On the left, $\left|V_{t^{\prime} d}\right| \approx\left|V_{L 4 u}\right|=0.04$. The red area is excluded by the constraints on $\left|V_{L 4 c}\right|$ from $D^{0}$ systems with $M_{t^{\prime}}=1 \mathrm{TeV}$, as reported in eq. (5.20). The red line shows where the boundary is shifted if the mass of the extra quark is taken as $M_{t^{\prime}}=$ $2.5 \mathrm{TeV}$. The blue area is excluded by neutral kaons mass difference, using the result $\left|V_{t^{\prime}}\right|<$ $1.4 \cdot 10^{-2}$ from eq. (5.23). The blue line indicates where the forbidden region expands if the mass of the extra quark is taken as $M_{t^{\prime}}=2.5 \mathrm{TeV}$. The green region is excluded by the relation in eq. (5.38), using the values for $V_{t^{\prime} b}$ allowed by the limit from the B-mesons system in eq. (5.31), figure 15 . On the right, the same constraints are shown setting $\left|V_{t^{\prime} d}\right| \approx\left|V_{L 4 u}\right|=0.03$ as a conservative benchmark value. The red and blue continuous (dotted) lines show where the boundaries from D-mesons and K-mesons shift if the mass of the extra quark is taken as $M_{t^{\prime}}=5 \mathrm{TeV}\left(M_{t^{\prime}}=2.5 \mathrm{TeV}\right)$. The dashed lines in both figures indicate the boundaries of the excluded area under more stringent assumptions. In particular, $\Delta_{K}=0.1$ is chosen in eq. (5.22) and the new contribution to D-mesons mass difference is limited as one third of the experimental value (4.101). 
However, in order to have the needed value $\left|V_{L 4 u}\right| \approx 0.043$, there is no much room to accommodate the relation in eq. (5.38) without contradicting experimental constraints from neutral mesons mixing. Some allowed region can be found for values $V_{L 4 u}<0.057$ with $M_{t^{\prime}}=1 \mathrm{TeV}$. In any case, the mass of the extra quark cannot exceed few $\mathrm{TeV}$. In fact, with larger values of $M_{t^{\prime}}$ constraints from neutral mesons mixing become more stringent (5.36), (5.37). In particular, as shown in figure 16 (left), for $\left|V_{L 4 u}\right| \simeq 0.04$ the mass of the extra quark should not exceed $\sim 2.5 \mathrm{TeV}$. Moreover, the constraint from K-mesons mixing in eq. (5.36) requires specific values of the phase $\delta_{d s}$ in order to avoid constraints from $\mathrm{CP}$ violation.

For $\left|V_{L 4 u}\right|=0.03$ (figure 16 right) the mass of the extra quark cannot exceed $\sim 5 \mathrm{TeV}$ in order to leave allowed values in the parameter space. If $M_{t^{\prime}} \approx 5 \mathrm{TeV}$, it should be that $\left|V_{t^{\prime} s}\right| \sim 3.8 \cdot 10^{-3},\left|V_{t^{\prime} b}\right| \sim 5.7 \cdot 10^{-3}$, meaning that in this case the mixing of $t^{\prime}$ with the second and third families is respectively eight times and five times smaller than the mixing with the d-quark. Moreover $\left|V_{t^{\prime} d}\right| \simeq 0.03$ is comparable to $\left|V_{c b}\right|$ and ten times larger than $\left|V_{u b}\right|$. This situation may seem unnatural, but it cannot be excluded.

The allowed parameter space can also be considerably reduced under more stringent assumptions. For example, dashed lines in figure 16 indicate the boundaries of the excluded area if $\Delta_{K}=0.1$ is chosen in eq. (5.22) and the new contribution to D-mesons mass difference is limited as one third of the experimental value (4.101). This means that precise knowledge could define the allowed area.

In addition to flavour changing constraints, the result for $Z$-boson decay rate into hadrons implies that (5.35):

$$
\left|V_{L 4 u}\right|^{2}<2.0 \cdot 10^{-3}, \quad\left|V_{L 4 u}\right|<0.044
$$

which is in the range of values needed to solve the CKM unitarity problem (for example, at $95 \%$ C.L. $\left|V_{L 4 u}\right|=0.043_{-0.015}^{+0.011}$ (4.8) using our conservative average for $V_{u d}(2.10)$ ). This means that also precision measurements of $\mathrm{Z}$ boson decay can exclude up-type weak singlet as a good solution of the CKM unitarity problem.

In any case, it should be $M_{t^{\prime}} \lesssim 5.8 \mathrm{TeV}$ with $\left|V_{t^{\prime} d}\right|=0.03$ and $M_{t^{\prime}} \lesssim 4.4 \mathrm{TeV}$ with $\left|V_{t^{\prime} d}\right|=0.04$ assuming for perturbativity $\left|h_{u 1}\right| \lesssim 1$.

\section{$6 \quad$ Extra weak isodoublet}

Weak isosinglets can be investigated in order to solve the problem of lack of unitarity in $V_{\text {CKM }}$ when $V_{u d}$ is extracted from superallowed beta decays and $V_{u s}$ from kaon decays. However, between the results from kaon physics there is inconsistency. In particular, it can be noticed that the lack of compatibility lies between vector and axial-vector couplings in weak charged currents. In fact, determination $B(2.13)$ is extracted from the axialvector coupling of leptonic kaon decays while determination $A(2.3)$ results from the vector coupling of semileptonic kaon decays. Besides, determination $C(2.14)$ is obtained from the vector coupling of beta decays. As displayed in figure 2, determination $A$ and $C$ are deviated from the axial-vector determination in opposite directions. In fact, there is $2.3 \sigma$ discrepancy between determination $B(2.13)$ and $C$ (2.14) and $3 \sigma$ discrepancy between 
determinations $A(2.3)$ and $B$. As it will be shown, the insertion of an extra vector-like isodoublet generates weak right currents which modify vector and axial-vector couplings thus offering the possibility to explain both the gaps. However, also in this case FCNC emerge at tree level and predictions for electroweak processes are modified, so experimental limits must be checked.

Let us introduce the additional vector-like SU(2)-doublet family:

$$
q_{L 4}=\left(\begin{array}{c}
u_{L 4} \\
d_{L 4}
\end{array}\right), \quad q_{R 4}=\left(\begin{array}{c}
u_{R 4} \\
d_{R 4}
\end{array}\right)
$$

New Yukawa couplings and mass terms should appear in the Lagrangian: $y_{i j}^{u \prime} \tilde{\varphi}{\overline{q_{L i}}}^{\prime} u_{R j}+$ $y_{i j}^{d \prime} \varphi{\overline{q_{L i}}}^{\prime} d_{R j}+m_{i}{\overline{q_{L i}}}^{\prime} q_{R 4}+$ h.c. with $i=1,2,3,4, j=1,2,3$. As regards the mass terms $m_{i} \overline{q_{L i}^{\prime}} q_{R 4}+$ h.c., since the four species of right-handed singlets $q_{L i}^{\prime}$ have identical quantum numbers, a unitary transformation can be applied on the four components $q_{L i}^{\prime}$ so that $m_{i}=0$ for $i=1,2,3$. Then the Yukawa couplings and the extra mass term are:

$$
\sum_{i=1}^{4} \sum_{j=1}^{3}\left[y_{i j}^{u} \tilde{\varphi} \overline{q_{L i}} u_{R j}+y_{i j}^{d} \varphi \overline{q_{L i}} d_{R j}\right]+M_{4 q} \overline{q_{L 4}} q_{R 4}+\text { h.c. }
$$

The down quark mass matrix looks like:

$$
\begin{aligned}
& \overline{d_{L i}} \mathbf{m}_{i j}^{(d)} d_{R j}+\text { h.c. }=
\end{aligned}
$$

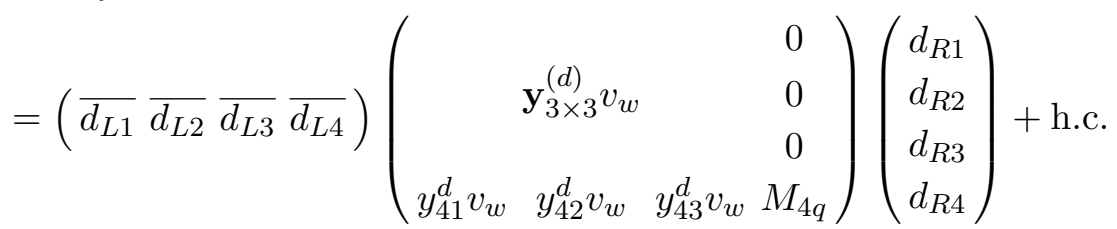

where $v_{w}=174 \mathrm{GeV}$ and $\mathbf{y}_{3 \times 3}^{(d)}$ is the $3 \times 3$ matrix of Yukawa couplings, and similarly for the up-type quarks. The mass matrices can be diagonalized with positive eigenvalues by biunitary transformations, as in (4.3) with mass eigenvalues $m_{d, s, b}=y_{d, s, b} v_{w}$ and $m_{u, c, t}=$ $y_{u, c, t} v_{w}$ and $M_{q} \approx M_{4 q} . V_{L, R}^{(d, u)}$ are unitary $4 \times 4$ matrices, as in (4.4) and analogously for up-type quarks. The charged-current Lagrangian is changed in:

$$
\begin{aligned}
\mathcal{L}_{c c}= & \frac{g}{\sqrt{2}} \sum_{i=1}^{4}\left(\overline{u_{L i}} \gamma^{\mu} d_{L i}\right) W_{\mu}^{+}+\frac{g}{\sqrt{2}} \overline{u_{4 R}} \gamma^{\mu} d_{4 R} W_{\mu}+\text { h.c. }= \\
= & \frac{g}{\sqrt{2}}\left(\overline{u_{L}} \overline{c_{L}} \overline{t_{L}} \overline{t_{L}^{\prime}}\right) \gamma^{\mu} V_{\mathrm{CKM}, \mathrm{L}}\left(\begin{array}{c}
d_{L} \\
s_{L} \\
b_{L} \\
b_{L}^{\prime}
\end{array}\right) W_{\mu}^{+}+\frac{g}{\sqrt{2}}\left(\overline{u_{R}} \overline{c_{R}} \overline{t_{R}} \overline{t_{R}^{\prime}}\right) \gamma^{\mu} V_{\mathrm{CKM}, \mathrm{R}}\left(\begin{array}{c}
d_{R} \\
s_{R} \\
b_{R} \\
b_{R}^{\prime}
\end{array}\right) W_{\mu}^{+} \\
& + \text {h.c. }
\end{aligned}
$$

where $V_{\mathrm{CKM}, \mathrm{L}}=V_{L}^{(u) \dagger} V_{L}^{(d)}$ is a $4 \times 4$ unitary matrix:

$$
\begin{aligned}
V_{\mathrm{CKM}, \mathrm{L}}^{\dagger} V_{\mathrm{CKM}, \mathrm{L}}=V_{\mathrm{CKM}, \mathrm{L}} V_{\mathrm{CKM}, \mathrm{L}}^{\dagger}=\mathbf{1} \\
V_{\mathrm{CKM}, \mathrm{L}}=V_{L}^{(u) \dagger} V_{L}^{(d)}=\left(\begin{array}{cccc}
V_{L u d} & V_{L u s} & V_{L u b} & V_{L u b^{\prime}} \\
V_{L c d} & V_{L c s} & V_{L c b} & V_{L c b^{\prime}} \\
V_{L t d} & V_{L t s} & V_{L t b} & V_{L t b^{\prime}} \\
V_{L t^{\prime} d} & V_{L t^{\prime} s} & V_{L t^{\prime} b} & V_{L t^{\prime} b^{\prime}}
\end{array}\right)
\end{aligned}
$$


In addition to that, in this case, as shown in eq. (6.4), the charged current Lagrangian $\mathcal{L}_{c c}$ also involves non-diagonal right weak charged currents originated by the mixing of the vector-like family with the SM families, with mixing matrix $V_{\mathrm{CKM}, \mathrm{R}}$ :

$$
\begin{aligned}
& V_{\mathrm{CKM}, \mathrm{R}}=V_{R}^{(u) \dagger} \operatorname{diag}(0,0,0,1) V_{R}^{(d)}= \\
& =\left(\begin{array}{cccc}
V_{R 4 u}^{*} V_{R 4 d} & V_{R 4 u}^{*} V_{R 4 s} & V_{R 4 u}^{*} V_{R 4 b} & V_{R 4 u}^{*} V_{R 4 b^{\prime}} \\
V_{R 4 c}^{*} V_{R 4 d} & V_{R 4 c}^{*} V_{R 4 s} & V_{R 4 c}^{*} V_{R 4 b} & V_{R 4 c}^{*} V_{R 4 b^{\prime}} \\
V_{R 4 t}^{*} V_{R 4 d} & V_{R 4 t}^{*} V_{R 4 s} & V_{R}^{*} V_{R} V_{R b} & V_{R 4 t}^{*} V_{R} 4 b^{\prime} \\
V_{R 4 t^{\prime}}^{*} V_{R 4 d} & V_{R 4 t^{\prime}}^{*} V_{R 4 s} & V_{R 4 t^{\prime}}^{*} V_{R 4 b} & V_{R 4 t^{\prime}}^{*} V_{R 4 b^{\prime}}
\end{array}\right)=\left(\begin{array}{cccc}
V_{R u d} & V_{R u s} & V_{R u b} & V_{R u b^{\prime}} \\
V_{R c d} & V_{R c s} & V_{R c b} & V_{R c b^{\prime}} \\
V_{R t d} & V_{R t s} & V_{R t b} & V_{R t b^{\prime}} \\
V_{R t^{\prime} d} & V_{R t^{\prime} s} & V_{R t^{\prime} b} & V_{R t^{\prime} b^{\prime}}
\end{array}\right)
\end{aligned}
$$

where $V_{R 4 \alpha}$ are the elements of the fourth row of the matrices $V_{R}^{(d)}, V_{R}^{(u)}$ and we defined the elements $V_{R \alpha \beta}$. Clearly, $V_{\mathrm{CKM}, \mathrm{R}}$ is not unitary.

Regarding the LH particles, the matrices $V_{L}^{(d)}, V_{L}^{(u)}$ can be parameterized with the same parameterization as in eq. (4.11). However, in this scenario mixings in the left-handed sector are much smaller and indeed negligible:

$$
\tilde{s}_{L i}^{u, d} \approx-\frac{y_{i}^{u, d} y_{4 i}^{u, d *} v_{w}^{2}}{M_{q}^{2}}
$$

where $y_{i}^{d}=y_{d, s, b}, y_{i}^{u}=y_{u, c, t}$, and $V_{L i j}^{(u, d)} y_{4 j}^{u, d} \approx y_{4 i}^{u, d}$. As regards the right-handed sector, without loss of generality, the basis can be chosen in which the mixing between the SM three families has been diagonalized and $V_{R}^{(d)}$ can be parameterized as:

$$
V_{R}^{(d)}=\left(\begin{array}{cccc}
V_{R 1 d} & V_{R 1 s} & V_{R 1 b} & V_{R 1 b^{\prime}} \\
V_{R 2 d} & V_{R 2 s} & V_{R 2 b} & V_{R 2 b^{\prime}} \\
V_{R 3 d} & V_{R 3 s} & V_{R 3 b} & V_{R 3 b^{\prime}} \\
V_{R 4 d} & V_{R 4 s} & V_{R 4 b} & V_{R 4 b^{\prime}}
\end{array}\right) \approx\left(\begin{array}{cccc}
c_{R 1}^{d} & 0 & 0 & -\tilde{s}_{R 1}^{d} \\
-\tilde{s}_{R 2}^{d} \tilde{s}_{R 1}^{d *} & c_{R 2}^{d} & 0 & -\tilde{s}_{R 2}^{d} \\
-\tilde{s}_{R 3}^{d} \tilde{s}_{R 1}^{d *} & -\tilde{s}_{R 2}^{d *} \tilde{s}_{R 3}^{d} & c_{R 3}^{d} & -\tilde{s}_{R 3}^{d} \\
\tilde{s}_{R 1}^{d *} & \tilde{s}_{R 2}^{d *} & \tilde{s}_{R 3}^{* d} & c_{R 1}^{d} c_{R 2}^{d} c_{R 3}^{d}
\end{array}\right)
$$

where $c_{R i}^{d}$ are cosines and $\tilde{s}_{R i}^{d}$ are complex sines of angles in the 14,24,34 family planes parameterizing the mixing of the first three families with the vector-like doublet, defined as in (4.12), and similarly for $V_{R}^{(u)}$. As it can be seen by comparing the mass matrix in eq. (6.3) with the matrix in eq. (4.2), in this case the angles parameterizing the mixing of the first three families with the fourth family in the right-handed sector are analogous in magnitude to the ones of the left-handed sector in the previous sections. The moduli of elements of the last row (and also of the last column) in $V_{R}^{(d)}$ and $V_{R}^{(u)}$ correspond to the mixing angles of the SM families with the vector-like one:

$$
V_{R 4 d} \approx \tilde{s}_{R 1}^{d *} \approx-\frac{y_{41}^{d} v_{w}}{M_{q}}, \quad V_{R 4 s} \approx \tilde{s}_{R 2}^{d *} \approx-\frac{y_{42}^{d} v_{w}}{M_{q}}, \quad V_{R 4 b}=\tilde{s}_{R 3}^{d *} \approx-\frac{y_{43}^{d} v_{w}}{M_{q}}
$$

and anagolously for up-type.

Let us focus on the piece of the charged current Lagrangian $\mathcal{L}_{c c}$ in eq. (6.4) determining the couplings of $u$-quark with down type quarks (which in the SM would correspond with the determination of the first row of CKM matrix):

$$
\begin{aligned}
& \frac{g}{\sqrt{2}}\left(\overline{u_{L}} \gamma^{\mu} V_{L u d} d_{L}+\overline{u_{R}} V_{R u d} \gamma^{\mu} d_{R}\right) W_{\mu}+\frac{g}{\sqrt{2}}\left(\overline{u_{L}} \gamma^{\mu} V_{L u s} s_{L}+\overline{u_{R}} V_{R u s} \gamma^{\mu} s_{R}\right) W_{\mu}+\text { h.c. }= \\
& =\frac{1}{2} \frac{g}{\sqrt{2}} \hat{V}_{u d} \bar{u} \gamma^{\mu}\left(1-\gamma^{5} k_{A}^{u d}\right) d W_{\mu}+\frac{1}{2} \frac{g}{\sqrt{2}} \hat{V}_{u s} \bar{u} \gamma^{\mu}\left(1-\gamma^{5} k_{A}^{u s}\right) s W_{\mu}
\end{aligned}
$$


where, from eq. (6.7):

$$
V_{R u d}=V_{R 4 u}^{*} V_{R 4 d}, \quad V_{R u s}=V_{R 4 u}^{*} V_{R 4 s}
$$

and similarly for the mixing with the bottom quark. The vector and axial-vector couplings are respectively: $\hat{V}_{u \alpha}=V_{L u \alpha}+V_{R u \alpha}$ and $k_{A}^{u \alpha} \hat{V}_{u \alpha}=V_{L u \alpha}-V_{R u \alpha}$, with $\alpha=d, s, b$. The most precise determination of SM $V_{u d}$ comes from superallowed beta decays. Superallowed $0^{+}-0^{+}$beta decays are Fermi transitions, that is they uniquely depend on the vector part of the hadronic weak interaction $G_{V}=G_{F} V_{u d}$. This means that the determination of the weak coupling in superallowed beta decays gives $\left|\hat{V}_{u d}\right|$ appearing in (6.11). Regarding $\left|V_{u s}\right|$, it is determined both from semileptonic kaon decays $(K \ell 3)$ and from the ratio of leptonic kaon decays $(K \mu 2)$ and leptonic pion decays. It is assumed that only the vector current contributes to semileptonic kaon decays, that is the coupling is given by $\hat{V}_{u s}$ in (6.11). Leptonic decays instead depend on the axial-vector coupling, which corresponds to $k_{A}^{u s} \hat{V}_{u s}$ in (6.11). Then, in this scenario, the determinations of $V_{u s}$ obtained from eqs. (2.3), (2.5), and (2.10) correspond to the following observables:

$$
\begin{array}{llrl}
A: & & \left|V_{\text {Lus }}+V_{\text {Rus }}\right| & =0.22326(55) \\
B: & & \frac{\left|V_{\text {Lus }}-V_{\text {Rus }}\right|}{\left|V_{\text {Lud }}-V_{\text {Rud }}\right|}=0.23130(49) \\
C: & & \left|V_{\text {Lud }}+V_{\text {Rud }}\right|=0.97362(26)
\end{array}
$$

As already stated (6.5), $V_{\mathrm{CKM}, \mathrm{L}}$ is a unitary matrix, so for the first row it holds that:

$$
\left|V_{L u d}\right|^{2}+\left|V_{L u s}\right|^{2}+\left|V_{L u b}\right|^{2}=1-\left|V_{L u b^{\prime}}\right|^{2} \approx 1
$$

In fact, $V_{L u b^{\prime}} \sim-\tilde{s}_{L 1}^{d}+\tilde{s}_{L 1}^{u} \sim O\left(y_{1}^{u, d} y_{41}^{u, d} v_{w}^{2} / M_{q}^{2}\right)$ is totally negligible, and also $\left|V_{L u b}\right|$ has almost no influence and the value of $\left|V_{u b}\right|$ as exctracted in the SM can be used. Hence, $\left|V_{L u d}\right|$ can be determined from $\left|V_{\text {Lus }}\right|$ by using the unitarity relation (6.16). Then, the system of the three different determinations A, B, C is exactly solved by three real parameters $V_{\text {Lus }}$, $V_{R u d}, V_{R u s}$. Using the dataset (6.13), (6.14), (6.15), the solution gives:

$$
V_{R u s}=V_{R 4 u}^{*} V_{R 4 s}=-1.17(37) \times 10^{-3}, \quad V_{R u d}=V_{R 4 u}^{*} V_{R 4 d}=-0.87(27) \times 10^{-3}
$$

with $V_{\text {Lus }}=0.22443(35)$ and $V_{\text {Lud }}=0.97448(8)$. Figure 17 basically shows the interpretation of the determinations of $V_{u s}$ obtained from the three different processes in the scenario with the extra isodoublet of quarks, as given in eqs. (6.13), (6.14), (6.15). The element of the unitary matrix $V_{\mathrm{CKM}, \mathrm{L}} V_{\text {Lus }}$ lies in between determinations $\mathrm{A}$ and $\mathrm{B}$, and the gap among the two is explained by the splitting due to $\pm V_{R u s}$ (the shift of determination $\mathrm{B}$ is dominated by $\left.V_{R u s}\right)$. On the other hand, $V_{R u d}$ explains the apparent lack of unitarity. In fact, the values of $V_{L u s}$ and $V_{L u d}$ are linked by the unitarity of $V_{\mathrm{CKM}, \mathrm{L}}$, but the vector coupling giving determination $\mathrm{C}$ includes the additional coupling $V_{R u d}$.

By substituting determination $\mathrm{C}$ with the determinations $C_{1}$ or $C_{2}$ (see table 1), the needed value of $V_{R u s}$ remains almost the same. Using determination $C_{1}:\left|V_{u d}\right|=$ $0.97355(27)$ with the dataset $(6.13),(6.14)$, the result would be $V_{R u s}=-1.18(37) \times 10^{-3}$, 


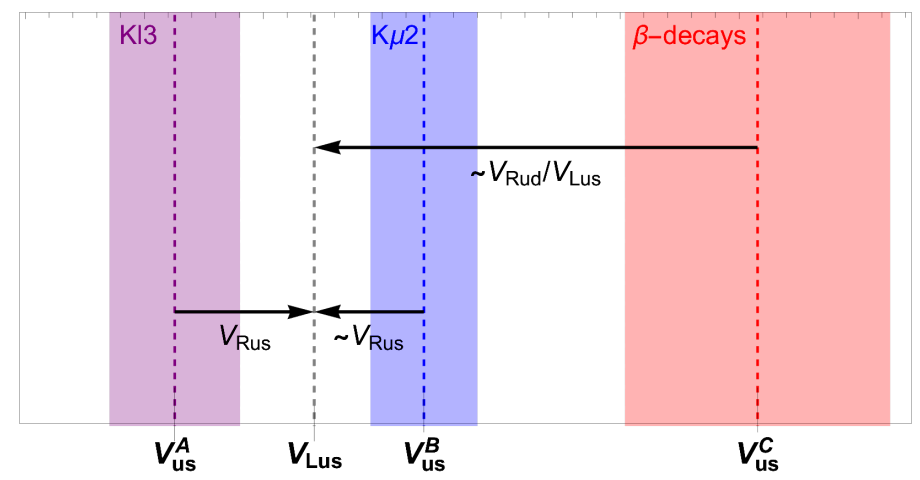

Figure 17. Explanation of the anomalies of $V_{u s}$ determinations in the SM extension with an extra vector-like isodoublet $\left(b^{\prime}, t^{\prime}\right)$. The three different determinations $V_{u s}^{A}, V_{u s}^{B}$ and $V_{u s}^{C}$ in eqs. (2.3), (2.13), (2.14) are obtained from the experimental results in eqs. (2.3), (2.5), (2.10) using CKM unitarity in the context of the SM. However, in presence of an extra isodoublet mixing with SM families, the three observables would correspond to the couplings in eqs. (6.13), (6.14), (6.15).

$V_{R u d}=-0.93(27) \times 10^{-3}$ with $V_{L u s}=0.22444(35)$, while using determination $C_{2}:\left|V_{u d}\right|=$ $0.97375(29)$, the solution is $V_{R u s}=-1.16(37) \times 10^{-3}, V_{R u d}=-0.74(29) \cdot 10^{-3}$ with $V_{\text {Lus }}=0.22441(35)$.

However also in this scenario flavour changing neutral currents appear at tree level. The neutral current interactions in terms of the mass eigenstates are described by the Lagrangian:

$$
\mathcal{L}_{\mathrm{nc}}=\frac{g}{\cos \theta_{W}} Z^{\mu}\left(g_{L}^{\alpha} \overline{q_{\alpha L}} \gamma_{\mu} q_{\alpha L}+g_{R}^{\alpha \beta} \overline{q_{\alpha R}} \gamma_{\mu} q_{\beta R}\right)
$$

with $q_{\alpha}=u, c, t, t^{\prime}, d, s, b, b^{\prime}$, and:

$$
g_{L}^{\alpha}=T_{3}^{\alpha}-Q_{\alpha} \sin ^{2} \theta_{W} \quad g_{R}^{\alpha \beta}=T_{3}^{\alpha} V_{R 4 \alpha}^{*} V_{R 4 \beta}-Q_{\alpha} \sin ^{2} \theta_{W} \delta_{\alpha \beta}
$$

where $T_{3}^{\alpha}$ is the weak isospin and $Q_{\alpha}$ is the charge in units of $e$. Clearly, FCNC arise from:

$$
\mathcal{L}_{\text {fcnc }}=\frac{1}{2} \frac{g}{\cos \theta_{W}} Z^{\mu}\left(\overline{u_{R 4}} \gamma_{\mu} u_{R 4}-\overline{d_{R 4}} \gamma_{\mu} d_{R 4}\right)=\frac{g}{\cos \theta_{W}} Z^{\mu} T_{3}^{\alpha} V_{R 4 \alpha}^{*} V_{R 4 \beta} \overline{q_{\alpha R}} \gamma_{\mu} q_{\beta R}
$$

Explicitly:

$$
\begin{aligned}
\mathcal{L}_{\mathrm{fcnc}}= & \frac{1}{2} \frac{g}{\cos \theta_{W}} Z^{\mu}\left(\overline{u_{R}} \overline{c_{R}} \overline{t_{R}} \overline{t_{R}^{\prime}}\right) \gamma^{\mu} V_{R}^{(u) \dagger} \operatorname{diag}(0,0,0,1) V_{R}^{(u)}\left(\begin{array}{c}
u_{R} \\
c_{R} \\
t_{R} \\
t_{R}^{\prime}
\end{array}\right)+ \\
& -\frac{1}{2} \frac{g}{\cos \theta_{W}} Z^{\mu}\left(\overline{d_{R}} \overline{s_{R}} \overline{b_{R}} \overline{b_{R}^{\prime}}\right) \gamma^{\mu} V_{R}^{(d) \dagger} \operatorname{diag}(0,0,0,1) V_{R}^{(d)}\left(\begin{array}{c}
d_{R} \\
s_{R} \\
b_{R} \\
b_{R}^{\prime}
\end{array}\right)
\end{aligned}
$$


where

$$
\begin{aligned}
V_{R}^{(u) \dagger} \operatorname{diag}(0,0,0,1) V_{R}^{(u)}= & \left(\begin{array}{cccc}
\left|V_{R 4 u}\right|^{2} & V_{R 4 u}^{*} V_{R 4 c} & V_{R 4 u}^{*} V_{R 4 t} & V_{R 4 u}^{*} V_{R 4 t^{\prime}} \\
V_{R 4 c}^{*} V_{R 4 u} & \left|V_{R 4 c}\right|^{2} & V_{R 4 c}^{*} V_{R 4 t} & V_{R 4 c}^{*} V_{R 4 t^{\prime}} \\
V_{R 4 t}^{*} V_{R 4 u} & V_{R 4 t}^{*} V_{R 4 c} & \left|V_{R 4 t}\right|^{2} & V_{R 4 t}^{*} V_{R 4 t^{\prime}} \\
V_{R 4 t^{\prime}}^{*} V_{R 4 u} & V_{R 4 t^{\prime}}^{*} V_{R 4 c} & V_{R 4 t^{\prime}}^{*} V_{R 4 t} & \left|V_{R 4 t^{\prime}}\right|^{2}
\end{array}\right) \\
V_{R}^{(d) \dagger} \operatorname{diag}(0,0,0,1) V_{R}^{(d)}= & \left(\begin{array}{cccc}
\left|V_{R 4 d}\right|^{2} & V_{R 4 d}^{*} V_{R 4 s} & V_{R 4 d}^{*} V_{R 4 b} & V_{R 4 d}^{*} V_{R 4 b^{\prime}} \\
V_{R 4 s}^{*} V_{R 4 d} & \left|V_{R 4 s}\right|^{2} & V_{R 4 s}^{*} V_{R 4 b} & V_{R 4 s}^{*} V_{R 4 b^{\prime}} \\
V_{R 4 b}^{*} V_{R 4 d} & V_{R 4 b}^{*} V_{R 4 s} & \left|V_{R 4 b}\right|^{2} & V_{R 4 b}^{*} V_{R 4 b^{\prime}} \\
V_{R 4 b^{\prime}}^{*} V_{R 4 d} & V_{R 4 b^{\prime}}^{*} V_{R 4 s} & V_{R 4 b^{\prime}}^{*} V_{R 4 b} & \left|V_{R 4 b^{\prime}}\right|^{2}
\end{array}\right)
\end{aligned}
$$

where, again, $V_{R 4 \alpha}$ are the elements of the fourth row of the matrices $V_{R}^{(d)}, V_{R}^{(u)}$.

\subsection{Limits from flavour changing neutral currents}

The new operators in eq. (6.21), due to the mixing of the extra vector-like quark isodoublet with SM families, give flavour changing processes at tree level. Therefore, they should be compared with experimental constraints. In particular, the hadronic vector current contribute to semileptonic mesons decays, while the hadronic axial-vector current gives leptonic mesons decays. By comparing the Lagrangian in eq. (6.21) and the matrices in eq. (6.22) with Lagrangians in eqs. (4.9), (5.7) and the matrix in eq. (4.10) (and the analogous for up-type), it can be noticed that the same effective operators are generated, with the substitution in the hadronic vector coupling:

$$
-V_{L 4 \alpha}^{*} V_{L 4 \beta} \rightarrow V_{R 4 \alpha}^{*} V_{R 4 \beta}
$$

and in the axial-vector coupling:

$$
V_{L 4 \alpha}^{*} V_{L 4 \beta} \rightarrow V_{R 4 \alpha}^{*} V_{R 4 \beta}
$$

It follows that there is a new contribution at tree level to the same flavour changing neutral current processes examined in the previous sections and the same analysis can be applied to the mixing elements of the matrices $V_{R}^{(u)}$ and $V_{R}^{(d)}$. In fact, aside from the substitution $L \rightarrow R$, only the sign of the interference in semileptonic decays is modified, that is only the phase dependence of the constraints from flavour changing semileptonic decays. In particular, upper limits from flavour changing kaon decays are summarized in figure 18 (which is the analogous to figure 11). In order to not contradict any bound, it results that:

$$
\left|V_{R 4 d} V_{R 4 s}^{*}\right| \lesssim(0.3 \div 1.3) \times 10^{-5}
$$

depending on the relative phase $\delta_{R 21}^{d}=\operatorname{Arg}\left(V_{R 4 d} V_{R 4 s}^{*}\right)$.

Concerning $K^{0}$ mixing, the new contribution from $\mathrm{RH}$ weak currents is:

$$
\begin{aligned}
\mathcal{L}_{\Delta S=2}^{\text {right }} & \approx-\frac{G_{F}}{\sqrt{2}}\left(V_{R 4 d}^{*} V_{R 4 s}\right)^{2}\left(1+\frac{G_{F} M_{q}^{2}}{4 \sqrt{2} \pi^{2}}\right)\left(\overline{d_{R}} \gamma^{\mu} s_{R}\right)^{2}+\text { h.c. }= \\
& =-\frac{G_{F}}{\sqrt{2}}\left(V_{R 4 d}^{*} V_{R 4 s}\right)^{2}\left[1+\left(\frac{M_{q}}{2.2 \mathrm{TeV}}\right)^{2}\right]\left(\overline{d_{R}} \gamma^{\mu} s_{R}\right)^{2}+\text { h.c. }
\end{aligned}
$$




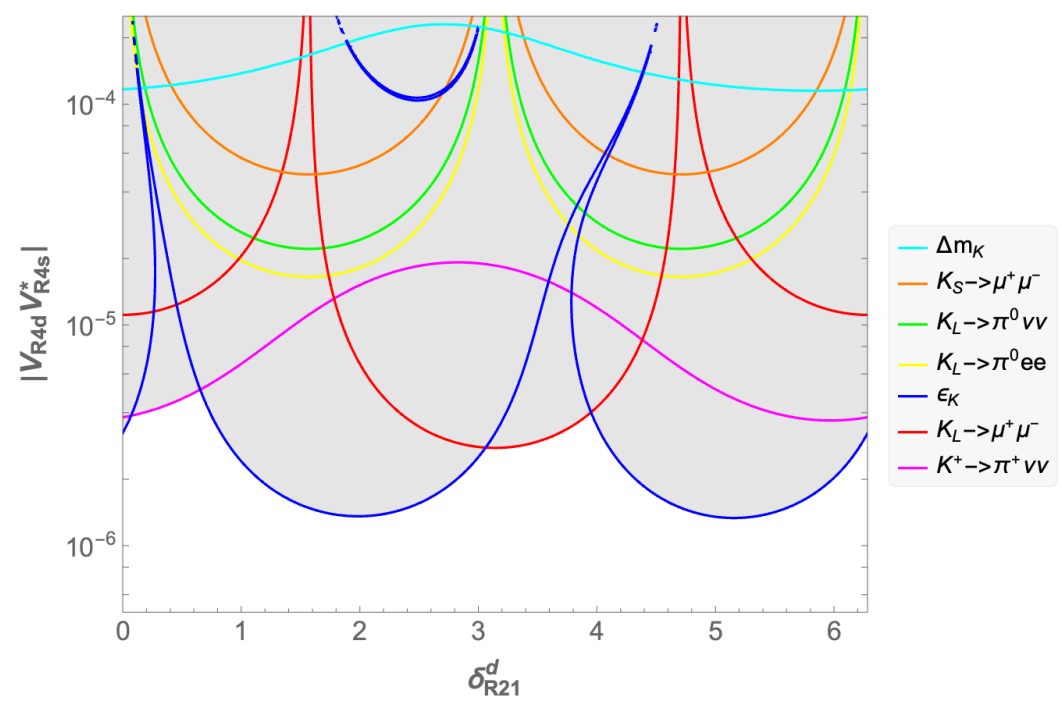

Figure 18. Upper limits on the product $\left|V_{R 4 s}^{*} V_{R 4 d}\right|$ from flavour changing kaon decays and neutral kaon mixing, as a function of the relative phase $\delta_{R 21}^{d}$, for the mass of the extra quarks $M_{q}=1 \mathrm{TeV}$.

However, in this case also mixed L-R contributions can in principle be relevant. In fact, the new contribution to the mixing mass term includes:

$$
\begin{aligned}
M_{12}^{\text {new }} \approx & \frac{1}{3} f_{K}^{2} m_{K^{0}} B_{K} \frac{G_{F}}{\sqrt{2}}\left(V_{R 4 d}^{*} V_{R 4 s}\right)^{2}+ \\
& +\frac{G_{F}^{2}}{4 \pi^{2}}\left[\frac{1}{2} M_{q}^{2}\left(V_{R 4 d}^{*} V_{R 4 s}\right)^{2}-27.5 m_{W}^{2} f\left(x_{q}, x_{t}\right) V_{R 4 d}^{*} V_{R 4 s} V_{t d}^{*} V_{t s}\right]
\end{aligned}
$$

where $x_{q}=M_{q}^{2} / m_{W}^{2}, x_{t}=m_{t}^{2} / m_{W}^{2}$, and $f\left(x_{q}, x_{t}\right) \approx 5$ for $M_{q}=1 \mathrm{TeV}$. Then, applying the constraints $\left|M_{12}^{\text {new }}\right|<\left|M_{12}^{\mathrm{SM}}\right|,\left|\operatorname{Im} M_{12}^{\text {new }}\right|<0.4\left|\operatorname{Im} M_{12}^{\mathrm{SM}}\right|$ (4.81), (4.85), for $M_{q}=1 \mathrm{TeV}$, as shown in figure 18, it is obtained:

$$
\left|V_{R 4 d}^{*} V_{R 4 s}\right|<\left(1.3 \times 10^{-6}\right) \div\left(2.2 \times 10^{-4}\right)
$$

depending on the relative phase of the elements. Together with the bounds from flavour changing kaon decays, in order to not contradict any limit:

$$
\left|V_{R 4 d} V_{R 4 s}^{*}\right| \lesssim(1.3 \div 5.1) \times 10^{-6}
$$

We can consider that, if eq. (6.17) should be satisfied, the product $V_{R 4 d}^{*} V_{R 4 s}$ should be real and positive (as can be seen by multiplying side by side the first equation with the complex conjugate of the second). Then the constraint from flavour changing processes of kaons would be:

$$
\left|V_{R 4 d}^{*} V_{R 4 s}\right|<3.2 \times 10^{-6}
$$

for $M_{q}=1 \mathrm{TeV}$.

The contribution to $D^{0}$ mesons system is:

$$
\mathcal{L}_{\Delta C=2}^{\text {new }} \approx-\frac{G_{F}}{\sqrt{2}}\left(V_{R 4 u}^{*} V_{R 4 c}\right)^{2}\left[1+\left(\frac{M_{q}}{2.2 \mathrm{TeV}}\right)^{2}\right]\left(\overline{u_{R}} \gamma^{\mu} c_{R}\right)^{2}+\text { h.c. }
$$


giving the condition analogous to the results in eqs. (5.20), (4.105):

$$
\left|V_{R 4 u}^{*} V_{R 4 c}\right|<1.3 \times 10^{-4}\left[\frac{f_{q}(1 \mathrm{TeV})}{f_{q}\left(M_{q}\right)}\right]^{1 / 2}\left[\frac{\Delta m_{D \max }}{8.2 \cdot 10^{-15}}\right]
$$

where

$$
f_{q}\left(M_{q}\right)=1+\left(\frac{M_{q}}{2.2 \mathrm{TeV}}\right)^{2}
$$

\subsection{Limits from $Z$-boson physics}

In this model the deviation of the $\mathrm{Z}$ decay rate from the $\mathrm{SM}$ prediction is:

$$
\begin{aligned}
& \Gamma(Z \rightarrow \text { had })-\Gamma(Z \rightarrow \text { had })_{\mathrm{SM}}=\Gamma(Z)-\Gamma(Z)_{\mathrm{SM}} \approx \\
& \approx \frac{G_{F} M_{Z}^{3}}{\sqrt{2} \pi}\left[-\frac{2}{3} \sin ^{2} \theta_{W}\left(\left|V_{R 4 u}\right|^{2}+\left|V_{R 4 c}\right|^{2}\right)-\frac{1}{3} \sin ^{2} \theta_{W}\left(\left|V_{R 4 d}\right|^{2}+\left|V_{R 4 s}\right|^{2}+\left|V_{R 4 b}\right|^{2}\right)\right]<0
\end{aligned}
$$

We can impose that this deviation is less than a chosen quantity $\Delta \Gamma_{Z}$ :

$$
\frac{G_{F} M_{Z}^{3}}{\sqrt{2} \pi}\left[\frac{2}{3} \sin ^{2} \theta_{W}\left(\left|V_{R 4 u}\right|^{2}+\left|V_{R 4 c}\right|^{2}\right)+\frac{1}{3} \sin ^{2} \theta_{W}\left(\left|V_{R 4 d}\right|^{2}+\left|V_{R 4 s}\right|^{2}+\left|V_{R 4 b}\right|^{2}\right)\right]<\Delta \Gamma_{Z}
$$

where QED+QCD corrections should also be included, that is a factor 1.050 for $u, c$-quarks, 1.041 for $d, s$-quarks and 1.021 for $b$-quark (there is additional correction for the bottom quark due to a loop with the $\mathrm{W}$ boson and the top quark) [20]. As shown in eq. (6.34) the prediction for the decay rate is lower than the SM expectation $\Gamma(Z \rightarrow \text { hadr })_{\mathrm{SM}}$. Then, since the SM expectation (4.141) is below the experimental result (4.142), we can choose to use the limit value of the $\mathrm{SM}$ prediction $\Gamma(Z \rightarrow \text { had })_{\mathrm{SM}}=1.7419 \mathrm{GeV}$ in eq. (6.35). Then, at $95 \%$ C.L. of the experimental result we can impose:

$$
\left|V_{R 4 u}\right|^{2}+\left|V_{R 4 c}\right|^{2}+0.5\left(\left|V_{R 4 d}\right|^{2}+\left|V_{R 4 s}\right|^{2}+\left|V_{R 4 b}\right|^{2}\right)<4.4 \times 10^{-3}\left[\frac{\Delta \Gamma_{Z}}{1.4 \times 10^{-3} \mathrm{GeV}}\right]
$$

This condition is compatible with the needed solution (6.17). However let us notice that, even setting $V_{R 4 c}=V_{R 4 b}=0$, this limit alone would rule out the possibility to explain the CKM anomalies with the extra weak doublet with a reduction of a factor less than 2 of the experimental error if the central values do not change. On the other hand, if the weak isodoublet is the solution to the CKM anomalies, anomolous $Z$-boson couplings with light fermions (in the "right" direction) should be detected if the error-bars are reduced by a factor $\gtrsim 5$.

As regards the couplings of $b$ and $c$ quarks, we can confront the new prediction with the experimental partial rates for the decays $Z \rightarrow b \bar{b}$ and $Z \rightarrow c \bar{c}$ using data from PDG [20], as reported in table 5 . At $95 \%$ C.L. they give the limits: $\left|V_{R 4 b}\right|<0.074,\left|V_{R 4 c}\right|<0.18$. 
As in previous sections, constraints are expected also from Z-pole asymmetry analyses of $e^{+} e^{-} \rightarrow f \bar{f}$. processes. The presence of an additional isodoublet changes the couplings of quarks with $Z$ boson and consequently the predictions for the asymmetries are changed as:

$$
A_{b}=\frac{1-\frac{4}{3} \bar{s}_{b}^{2}\left(1-\left|V_{R 4 b}\right|^{2}\right)-\left|V_{R 4 b}\right|^{4}}{1-\frac{4}{3} \bar{s}_{b}^{2}\left(1+\left|V_{R 4 b}\right|^{2}\right)+\frac{8}{9} \bar{s}_{b}^{4}+\left|V_{R 4 b}\right|^{4}}, \quad A_{c}=\frac{1-\frac{8}{3} \bar{s}_{c}^{2}\left(1-\left|V_{R 4 c}\right|^{2}\right)-\left|V_{R 4 c}\right|^{4}}{1-\frac{8}{3} \bar{s}_{c}^{2}\left(1+\left|V_{R 4 c}\right|^{2}\right)+\frac{32}{9} \bar{s}_{c}^{4}+\left|V_{R 4 c}\right|^{4}}
$$

where $\bar{s}_{f}^{2}$ are the effective weak angles which take into account EW radiative corrections. Taking the data from Particle Data Group [20], also reported in table 5, regarding b-quark final state, the mixing of the extra-doublet increases the prediction of $A_{b}$, thus not going to the "right" direction with respect to the experimental determinations of $A_{b}$ and especially of $A_{F B}^{(0, b)}$. However, with $V_{R 4 b}<0.19$ the expected value of $A_{b}$ remains in the $95 \%$ C.L. interval of the experimental result $A_{b}=0.923 \pm 0.020$.

Similarly, as regards c-quark final state, the prediction for $A_{c}$ is increased by the mixing of the extra-doublet. However, the expected value of $A_{c}$ remains in the $95 \%$ C.L. interval of the experimental determination $A_{c}=0.670 \pm 0.027$ with $V_{R 4 c}<0.18$. and it also stands in the $95 \%$ C.L. interval of the determination $A_{c}=0.628 \pm 0.032$ (which can be obtained from $A_{F B}^{(0, c)}=0.0707 \pm 0.0035$ using $\left.A_{e}=0.1501 \pm 0.0016\right)$ with $V_{R 4 c}<0.11$.

Constraints from Z-boson physics are summarized in table 8.

\subsection{Limits from low energy electroweak observables}

The insertion of the extra isodoublet also changes the diagonal couplings of weak neutralcurrent interactions, as follows from eqs. (6.18) and (6.19). Then, predictions on low energy electroweak precision observables are modified. At low momentum transfer $\left(Q^{2} \ll M_{Z}^{2}\right)$, the parity violating part of four-fermion Lagrangian corresponding to $e$-hadron processes with Z-boson exchange is written as [20]:

$$
\mathcal{L}=\frac{G_{F}}{\sqrt{2}} \sum_{q}\left[g_{A V}^{e q} \bar{e} \gamma_{\mu} \gamma^{5} e \bar{q} \gamma^{\mu} q+g_{V A}^{e q} \bar{e} \gamma_{\mu} e \bar{q} \gamma^{\mu} \gamma^{5} q\right]
$$

where in this case:

$$
\begin{array}{ll}
g_{A V}^{e u}=-\frac{1}{2}\left(1+\left|V_{R 4 u}\right|^{2}\right)+\frac{4}{3} \sin ^{2} \theta_{W} & g_{A V}^{e d}=\frac{1}{2}\left(1+\left|V_{R 4 d}\right|^{2}\right)-\frac{2}{3} \sin ^{2} \theta_{W} \\
g_{V A}^{e u}=\left(-\frac{1}{2}+2 \sin ^{2} \theta_{W}\right)\left(1-\left|V_{R 4 u}\right|^{2}\right) & g_{V A}^{e d}=-\left(-\frac{1}{2}+2 \sin ^{2} \theta_{W}\right)\left(1-\left|V_{R 4 d}\right|^{2}\right)
\end{array}
$$

The weak charge of the proton, $Q_{W}^{p}$, is proportional to $g_{A V}^{e p}=2 g_{A V}^{e u}+g_{A V}^{e d}, Q_{W}^{p}=$ $-2 g_{A V}^{e p}$. In the SM we have $g_{A V, \mathrm{SM}}^{e p}=-0.0357$ (where, after including higher orders corrections, $\left.g_{A V, \mathrm{SM}}^{e u}=-0.1888, g_{A V, \mathrm{SM}}^{e d}=0.3419[20]\right)$. Experimentally, the weak charge of the proton can be extracted from the parity violating right-left asymmetry in $e^{-} p \rightarrow e^{-} p$ scattering, from which it is obtained the constraint [20]:

$$
g_{A V, \exp }^{e p}=2 g_{A V}^{e u}+g_{A V}^{e d}=0.0356 \pm 0.0023
$$


which is in agreement with the SM expectation. After considering the existence of the extra isodoublet, the expected weak charge changes. In order to stay in the 95\% C.L. of the experimental value, it should be:

$$
\left|\Delta g_{A V}^{e p}\right|=\left.|-| V_{R 4 u}\right|^{2}+\frac{1}{2}\left|V_{R 4 d}\right|^{2} \mid<0.0045
$$

where $\Delta g_{A V}^{e p}$ is the additional contribution to $g_{A V, \mathrm{SM}}^{e p}$. Nuclear weak charges $Q_{W}^{Z, N}$ can be extracted from measurements of atomic parity violation. They are defined as [20]:

$$
Q_{W}^{Z, N}=-2\left[Z\left(g_{A V}^{e p}+0.00005\right)+N\left(g_{A V}^{e n}+0.00006\right)\right]\left(1-\frac{\alpha}{2 \pi}\right)
$$

where $Z$ and $N$ are the numbers of protons and neutrons in the nucleus, $g_{A V}^{e p}=2 g_{A V}^{e u}+g_{A V}^{e d}$, $g_{A V}^{e n}=g_{A V}^{e u}+2 g_{A V}^{e d}$, and $\alpha$ is the fine structure constant, $\alpha^{-1} \approx 137.036$. The most precise measurement of atomic parity violation is in Cesium [20]:

$$
Q_{W}^{55,78}(C s)_{\exp }=-72.82 \pm 0.42
$$

corresponding to the constraint $55 g_{A V}^{e p}+78 g_{A V}^{e n}=36.45 \pm 0.21$, while the SM prediction is $Q_{W}^{55,78}(C s)_{\mathrm{SM}}=-73.23 \pm 0.01\left(55 g_{A V}^{e p}+78 g_{A V}^{e n}=36.65\right)$. The contribution of the extra isodoublet to the weak charge of cesium is:

$$
\Delta Q_{W}^{55,78}(C s)=Q_{W}^{55,78}(C s)_{\mathrm{tot}}-Q_{W}^{55,78}(C s)_{\mathrm{SM}} \approx-2\left[-94\left|V_{R 4 u}\right|^{2}+105.5\left|V_{R 4 d}\right|^{2}\right]
$$

At $95 \%$ C.L. we have the condition:

$$
-0.0022\left[\frac{\left|\Delta Q_{W}(C s)\right|}{0.41}\right]<\left|V_{R 4 u}\right|^{2}-1.12\left|V_{R 4 d}\right|^{2}<0.0066\left[\frac{\Delta Q_{W}(C s)}{1.23}\right]
$$

Other neutral current parameters include [20]:

$$
\begin{aligned}
& \left(g_{A V}^{e u}+2 g_{A V}^{e d}\right)_{\exp }=0.4927 \pm 0.0031 \\
& \left(2 g_{A V}^{e u}-g_{A V}^{e d}\right)_{\exp }=-0.7165 \pm 0.0068
\end{aligned}
$$

to be compared with the SM expectations $g_{A V}^{e u}+2 g_{A V}^{e d}=0.4950,2 g_{A V}^{e u}-g_{A V}^{e d}=-0.7195$. Regarding the quantity $g_{A V}^{e u}+2 g_{A V}^{e d}$, the mixing with the extra doublet brings an extra contribution $-\frac{1}{2}\left|V_{R 4 u}\right|^{2}+\left|V_{R 4 d}\right|^{2}$. Then, at $95 \%$ C.L. we obtain the constraint:

$$
-0.0084<-\frac{1}{2}\left|V_{R 4 u}\right|^{2}+\left|V_{R 4 d}\right|^{2}<0.0038
$$

As regards the quantity $2 g_{A V}^{e u}-g_{A V}^{e d}$, the prediction is lowered with the extra doublet by $-\left|V_{R 4 u}\right|^{2}-\frac{1}{2}\left|V_{R 4 d}\right|^{2}$. Then, in the $2 \sigma$ interval we have:

$$
\left|V_{R 4 u}\right|^{2}+\frac{1}{2}\left|V_{R 4 d}\right|^{2}<0.010
$$

Constraints from low energy electroweak observables are summarized in table 8 . 


\begin{tabular}{|ll|}
\hline Process & Constraint \\
\hline$Z \rightarrow$ hadrons, $\Gamma_{Z}$ & $\left|V_{R 4 u}\right|^{2}+\left|V_{R 4 c}\right|^{2}+0.5\left(\left|V_{R 4 d}\right|^{2}+\left|V_{R 4 s}\right|^{2}+\left|V_{R 4 b}\right|^{2}\right)<4.4 \times 10^{-3}$ \\
$Q_{W}(C s)$ & $-0.0022<\left|V_{R 4 u}\right|^{2}-1.12\left|V_{R 4 d}\right|^{2}<0.0066$ \\
$Q_{W}(p)$ & $\left.|-| V_{R 4 u}\right|^{2}+\frac{1}{2}\left|V_{R 4 d}\right|^{2} \mid<0.0045$ \\
$g_{A V}^{e u}+2 g_{A V}^{e d}$ & $-0.0084<-\frac{1}{2}\left|V_{R 4 u}\right|^{2}+\left|V_{R 4 d}\right|^{2}<0.0038$ \\
$2 g_{A V}^{e u}-g_{A V}^{e d}$ & $\left|V_{R 4 u}\right|^{2}+\frac{1}{2}\left|V_{R 4 d}\right|^{2}<0.010$ \\
\hline
\end{tabular}

Table 8. Limits on the mixing of the first three families with an extra vector-like isodoublet from low energy electroweak observables and Z physics. For details see the text.

\subsection{Summary of experimental limits}

In this section we analyzed the scenario in which an extra vector-like weak doublet of quarks $\left(u_{4}, d_{4}\right)_{L, R}$ is mixing with the SM families, with mixing matrices $V_{R}^{(d, u)}(6.9)$. Although the presence of this extra species can in principle address the whole problem of CKM anomalies, it is not possible to obtain an acceptable solution satisfying both eq. (6.17) and the experimental constraints from kaons flavour changing processes (figure 18) and electroweak observables (table 8) by introducing one extra isodoublet of quarks.

In order to show this, we can focus on the elements $V_{R 4 u}, V_{R 4 d}, V_{R 4 s}$ of the mixing matrices $V_{R}^{(d, u)}$, which are the elements affecting the first row of CKM and consequently CKM anomalies (for our concern the other mixing elements can also be zero). Summarizing all the results, the mixings of the fourth doublet of quarks with SM families should be large enough in order to justify the anomalies between the different determinations of $V_{u s}$, as found in eq. (6.17):

$$
\left|V_{R u d}\right|=\left|V_{R 4 u}^{*} V_{R 4 d}\right|=0.87(27) \times 10^{-3}, \quad\left|V_{R u s}\right|=\left|V_{R 4 u}^{*} V_{R 4 s}\right|=1.17(37) \times 10^{-3}
$$

but at the same time they should not contradict the constraints from FCNC in kaon decays and mixing in eq. (6.29) and from low energy electroweak quantities and $Z$-boson physics in table 8:

$$
\left|V_{R 4 u}\right|^{2}+0.50\left(\left|V_{R 4 d}\right|^{2}+\left|V_{R 4 s}\right|^{2}\right)<4.4 \times 10^{-3}, \quad\left|V_{R 4 d} V_{R 4 s}^{*}\right| \lesssim(1.3 \div 5.1) \times 10^{-6}
$$

where the first constraint comes from the decay rate of $Z$-boson into hadrons. Let us underline that flavour changing conditions are obtained for the extra quarks mass $M_{q}=$ $1 \mathrm{TeV}$ and they become more stringent for larger masses. Figure 19 shows the excluded area in $V_{R 4 u}, V_{R 4 d}$ parameter space, after adopting some conservative choices. In particular, as it will be described below, it is shown that even by choosing the very conservative condition $\left|V_{R 4 u}^{*} V_{R 4 s}\right|=0.8 \times 10^{-3}$ and an indicative bound $\left|V_{R 4 d} V_{R 4 s}^{*}\right|<1.0 \cdot 10^{-5}$, there remains no allowed area in the parameter space.

The black curve shows the condition (6.17) $\left|V_{R u d}\right|=\left|V_{R 4 u}^{*} V_{R 4 d}\right|=0.87(27) \times 10^{-3}$, obtained as solution of the dataset (6.13), (6.14), (6.15).

The orange area is excluded by each bound from low energy electroweak quantities, as reported in table 8. Then, we should consider the strong restrictions coming from 


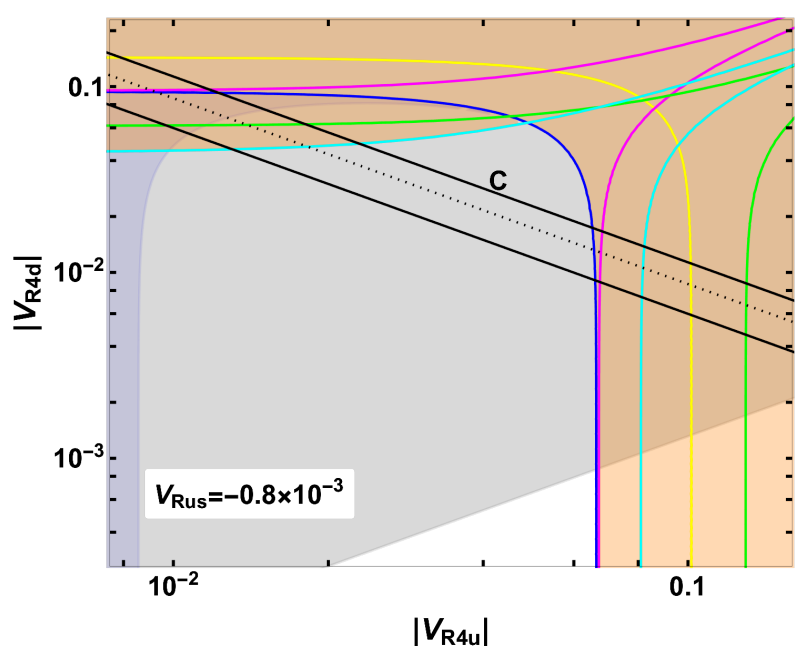

Figure 19. Excluded area in the $\left|V_{R 4 d}\right|$ and $\left|V_{R 4 u}\right|$ parameter space. The orange area is excluded by the following low energy electroweak quantities: $Z$-boson decay rate (blue contour), $Q_{W}(p)$ (magenta), $Q_{W}(C s)$ (cyan), the couplings $g_{A V}^{e u}+2 g_{A V}^{e d}$ (green) and $2 g_{A V}^{e u}-g_{A V}^{e d}$ (yellow). The black curve is the needed solution $\left|V_{R u d}\right|=\left|V_{R 4 u}^{*} V_{R 4 d}\right|=0.87(27) \times 10^{-3}$ in eq. (6.17). A conservative value $\left|V_{R u s}\right|=\left|V_{R 4 u}^{*} V_{R 4 s}\right|=0.8 \times 10^{-3}$ is used in order to satisfy the second relation of the solution in eq. (6.17). Using this value, the blue area is excluded by the experimental determination of $Z$ decay rate, and the gray area is excluded by the constraint from flavour changing kaon processes, using the indicative bound $\left|V_{R 4 d} V_{R 4 s}^{*}\right|<1.0 \cdot 10^{-5}$ for the mass of the extra quark $M_{q}=1 \mathrm{TeV}$.

flavor changing kaon decays. As obtained in eq. (6.25), the result is that the product $\left|V_{R 4 d} V_{R 4 s}^{*}\right|$ cannot exceed the limit $\left|V_{R 4 d} V_{R 4 s}^{*}\right| \lesssim 10^{-5}$, which is obtained for specific values of the relative phase $\delta_{R 21}^{d}=\operatorname{Arg}\left(V_{R 4 d} V_{R 4 s}^{*}\right)$, and the condition would be more stringent considering constraints from neutral kaon mixing. On the other hand, the condition $V_{R u s}=$ $V_{R 4 u}^{*} V_{R 4 s}=-1.17(37) \times 10^{-3}$ is needed in order to explain the discrepancy between the determinations of $V_{u s}$ from leptonic and semileptonic kaon decays. In figure 19 we show the excluded area (gray) adopting the conservative choices:

$$
\left\{\begin{array}{l}
V_{R 4 u}^{*} V_{R 4 s}=-0.8 \times 10^{-3} \\
\left|V_{R 4 d} V_{R 4 s}^{*}\right| \lesssim 1.0 \times 10^{-5}
\end{array}\right.
$$

These conservative choices also imply $\left|V_{R 4 u}\right|=\left|V_{R u d} V_{R u s}\right| /\left|V_{R 4 d} V_{R 4 s}\right| \gtrsim 0.2$, which is a limit value for perturbativity considering that (6.10) $V_{R 4 u} \approx-\frac{y_{41}^{u} v_{w}}{M_{q}}, M_{q} \gtrsim 700 \mathrm{GeV}$. However, such a value largely contradicts $\mathrm{Z}$ decay experimental results. In fact, with the same conservative choice for $V_{R u s}$, the blue area in figure 19 is excluded in order to have a solution not contradicting experimental measurements of hadronic $Z$ decay rate:

$$
\left\{\begin{array}{l}
V_{R 4 u}^{*} V_{R 4 s}=-0.8 \times 10^{-3} \\
\left|V_{R 4 u}\right|^{2}+0.50\left(\left|V_{R 4 d}\right|^{2}+\left|V_{R 4 s}\right|^{2}\right)<4.4 \times 10^{-3}
\end{array}\right.
$$

It is clear that the values needed as solutions are unachievable without contradiction with flavor changing experimental limits. 
Namely, by performing a fit of the values in eqs. (2.3), (2.5), (2.10), with real parameters $V_{L u s}, V_{R 4 d}, V_{R 4 s}, V_{R 4 u}$, but constraining them with experimental limits from flavour changing kaon decays and $Z$ decay into hadrons, the best fit point $\left(\chi^{2}=9\right)$ is obtained in $V_{\text {Lus }}=0.22464$ with:

$$
V_{R 4 u}=6.6 \times 10^{-2} \quad V_{R 4 d}=-1.1 \times 10^{-2} \quad V_{R 4 s}=-0.98 \times 10^{-3}
$$

and consequently

$$
V_{R u s}=V_{R 4 u}^{*} V_{R 4 s}=-0.65 \cdot 10^{-4} \quad V_{R u d}=V_{R 4 u}^{*} V_{R 4 d}=-0.71 \cdot 10^{-3}
$$

In any case, in order to not violate FC constraints only one of the two discrepancies, between determination B (2.13) and determination A (2.3) or between determination B and determination $\mathrm{C}$ (2.14), can be solved.

Moreover, the LHC limit on extra vector-like quarks coupling to light quarks $M_{q} \gtrsim$ $700 \mathrm{GeV}$ [82] implies that $\left|V_{R 4 u}\right| \simeq 0.066$ can be obtained if $\left|y_{41}^{u}\right| \sim 0.27$, much larger than the Yukawa constant of the bottom quark. Further, with $\left|V_{R 4 u}\right| \sim 0.066$, assuming (for the perturbativity) $\left|y_{41}^{u}\right| \lesssim 1$, there is an upper limit on the extra doublet mass, $M_{q} \lesssim 3 \mathrm{TeV}$.

\subsection{Possible extensions}

Looking at the results of previous sections, we can conclude that the presence of one single extra species cannot explain all the CKM anomalies without contradicting other experimental constraints, in particular from flavour changing processes of kaons and $\mathrm{Z}$ decay rate into hadrons. Then, in order to explain the Cabibbo angle anomalies, it can be considered the case in which for example there exist two or more vector-like doublets mixing with SM families. The Yukawa couplings and the mass terms can be written in the following basis:

$$
\sum_{i=1}^{5} \sum_{j=1}^{3}\left[y_{i j}^{u} \tilde{\varphi} \overline{q_{L i}} u_{R j}+y_{i j}^{d} \varphi \overline{q_{L i}} d_{R j}\right]+M_{4} \overline{q_{L 4}} q_{R 4}+M_{5} \overline{q_{L 5}} q_{R 5}+\text { h.c. }
$$

Then, in order to avoid flavour changing effects, not all the couplings $y_{i j}^{u, d}$ should be nonzero. Let us suppose that there can be a pattern of couplings, so that the first doublet has negligible coupling with the second down-type flavour: $y_{41}^{d} \neq 0, y_{42}^{d}=0, y_{41}^{u} \neq 0$, while the second doublet has negligible coupling with the first down quark: $y_{51}^{d}=0, y_{52}^{d} \neq 0$, $y_{51}^{u} \neq 0$. For simplicity, we also take $y_{43}^{d}, y_{53}^{d}=0$ and $y_{4 i}^{u}, y_{5 i}^{u}=0$ for $i \neq 1$. Then $V_{L, R}^{(d, u)}$ diagonalizing the mass matrices are unitary $5 \times 5$ matrices.

The charged-current Lagrangian is changed in:

$$
\begin{aligned}
\mathcal{L}_{c c}= & \frac{g}{\sqrt{2}} \sum_{i=1}^{5}\left(\overline{u_{L i}} \gamma^{\mu} d_{L i}\right) W_{\mu}^{+}+\frac{g}{\sqrt{2}} \overline{u_{4 R}} \gamma^{\mu} d_{4 R} W_{\mu}+\frac{g}{\sqrt{2}} \overline{u_{5 R}} \gamma^{\mu} d_{5 R} W_{\mu}+\text { h.c. }= \\
= & \frac{g}{\sqrt{2}} W_{\mu}^{+}\left[\left(\overline{u_{L}} \overline{c_{L}} \overline{t_{L}} \overline{t_{L}^{\prime}} \overline{t_{L}^{\prime \prime}}\right) \gamma^{\mu} V_{\mathrm{CKM}, \mathrm{L}}\left(\begin{array}{c}
d_{L} \\
s_{L} \\
b_{L} \\
b_{L}^{\prime} \\
b_{L}^{\prime \prime}
\end{array}\right)+\left(\overline{u_{R}} \overline{c_{R}} \overline{t_{R}} \overline{t_{R}^{\prime}} \overline{t_{R}^{\prime \prime}}\right) \gamma^{\mu} V_{\mathrm{CKM}, \mathrm{R}}\left(\begin{array}{c}
d_{R} \\
s_{R} \\
b_{R} \\
b_{R}^{\prime} \\
b_{R}^{\prime \prime}
\end{array}\right)\right] \\
& + \text { h.c. }
\end{aligned}
$$


where $V_{\mathrm{CKM}, \mathrm{L}}$ is unitary and the mixings of the first three famillies with the vector-like isodoublets are negligibly small, while in this case $V_{\mathrm{CKM}, \mathrm{R}}$ is given by:

$$
\begin{aligned}
& V_{\mathrm{CKM}, \mathrm{R}}=V_{R}^{(u) \dagger} \operatorname{diag}(0,0,0,1,0) V_{R}^{(d)}+V_{R}^{(u) \dagger} \operatorname{diag}(0,0,0,0,1) V_{R}^{(d)} \approx \\
& \approx\left(\begin{array}{ccccc}
V_{R 4 u}^{*} V_{R 4 d} & V_{R 5 u}^{*} V_{R 5 s} & 0 & V_{R 4 u}^{*} V_{R 4 b^{\prime}} & V_{R 5 u}^{*} V_{R 5 b^{\prime \prime}} \\
0 & 0 & 0 & 0 & 0 \\
0 & 0 & 0 & 0 & 0 \\
V_{R 4 t^{\prime}}^{*} V_{R 4 d} & 0 & 0 & V_{R 4 t^{\prime}}^{*} V_{R 4 b^{\prime}} & 0 \\
0 & V_{R 5 t^{\prime \prime}}^{*} V_{R 5 s} & 0 & 0 & V_{R 5 t^{\prime \prime}}^{*} V_{R 5 b^{\prime \prime}}
\end{array}\right)
\end{aligned}
$$

Then, the condition (6.17) is requiring:

$$
V_{R 5 u}^{*} V_{R 5 s}=-1.17(37) \times 10^{-3} \quad V_{R 4 u}^{*} V_{R 4 d}=-0.87(27) \times 10^{-3}
$$

The additional terms in the neutral current Lagrangian are:

$$
\begin{aligned}
\mathcal{L}_{\mathrm{fcnc}} & =\frac{1}{2} \frac{g}{\cos \theta_{W}} Z^{\mu}\left(\overline{u_{R 4}} \gamma_{\mu} u_{R 4}-\overline{d_{R 4}} \gamma_{\mu} d_{R 4}+\overline{u_{R 5}} \gamma_{\mu} u_{R 5}-\overline{d_{R 5}} \gamma_{\mu} d_{R 5}\right)= \\
& =\frac{1}{2} \frac{g}{\cos \theta_{W}} Z^{\mu}\left[\left(\overline{u_{R}} \overline{c_{R}} \overline{t_{R}} \overline{t_{R}^{\prime}} \overline{t_{R}^{\prime \prime}}\right) \gamma^{\mu} V_{\mathrm{nc}}^{(u)}\left(\begin{array}{c}
u_{R} \\
c_{R} \\
t_{R} \\
t_{R}^{\prime} \\
t_{R}^{\prime \prime}
\end{array}\right)-\left(\overline{d_{R}} \overline{s_{R}} \overline{b_{R}} \overline{b_{R}^{\prime}} \overline{b_{R}^{\prime \prime}}\right) \gamma^{\mu} V_{\mathrm{nc}}^{(d)}\left(\begin{array}{c}
d_{R} \\
s_{R} \\
b_{R} \\
b_{R}^{\prime} \\
b_{R}^{\prime \prime}
\end{array}\right)\right]
\end{aligned}
$$

where in this case the matrix $V_{\mathrm{nc}}^{(d)}$ is:

$$
\begin{aligned}
V_{\mathrm{nc}}^{(d)} & =V_{R}^{(d) \dagger} \operatorname{diag}(0,0,0,1,1) V_{R}^{(d)} \approx \\
& \approx\left(\begin{array}{ccccc}
\left|V_{R 4 d}\right|^{2} & 0 & 0 & V_{R 4 d}^{*} V_{R 4 b^{\prime}} & 0 \\
0 & \left|V_{R 5 s}\right|^{2} & 0 & 0 & V_{R 5 s}^{*} V_{R 5 b^{\prime \prime}} \\
0 & 0 & 0 & 0 & 0 \\
V_{R 4 b^{\prime}}^{*} V_{R 4 d} & 0 & 0 & \left|V_{R 4 b^{\prime}}\right|^{2} & 0 \\
0 & V_{R 5 b^{\prime \prime}}^{*} V_{R 5 s} & 0 & 0 & \left|V_{R 5 b^{\prime \prime}}\right|^{2}
\end{array}\right)
\end{aligned}
$$

Therefore, at first order there are no FCNC between the first two SM families. Then, the solution explaining the anomalies in the first row of CKM matrix in eq. (6.17), and equivalently in eq. (6.60), can be obtained without contradiction with experimental constraints on flavour changing phenomena. Regarding flavour conserving observables, the constraint from $Z$-boson decay into hadrons gives (6.36):

$$
\left|V_{R 4 u}\right|^{2}+\left|V_{R 5 u}\right|^{2}+0.50\left(\left|V_{R 4 d}\right|^{2}+\left|V_{R 5 s}\right|^{2}\right)<4.4 \times 10^{-3}
$$

which can be satisfied together with the relations in eq. (6.60). However, let us notice that a reduction of the experimental error by a factor less than 2 , with the same central values, would rule out the possibility to explain all the anomalies with this kind of solution. On 
the other hand, if the weak isodoublets are the solution to the CKM anomalies, anomolous $Z$-boson couplings with light fermions (in the "right" direction) should be detected if the error-bars are reduced by a factor of about $\gtrsim 4$.

Again, the LHC limit on the mass extra vector-like quark mixing with light families $M \gtrsim 700 \mathrm{GeV}[82]$ implies that $\left|V_{R 4 d}\right|,\left|V_{R 5 s}\right|,\left|V_{R 4,5 u}\right| \sim 0.03$ can be obtained if $\left|y_{i j}^{u, d}\right| \sim$ 0.12, much larger than the bottom Yukawa coupling. Moreover, in order to have (for the perturbativity) $\left|y_{i j}^{u, d}\right|<1$, the mass of the extra quarks should be no more than $M_{4,5} \sim 6 \mathrm{TeV}$.

Alternatively it can be imagined that there exist a vector-like isodoublet together with a down-type or up-type isosinglet, or both of them, assembling a complete vector-like fourth family with the doublet, mixing with SM families:

$$
q_{R 4}=\left(\begin{array}{c}
u_{R 4} \\
d_{R 4}
\end{array}\right), q_{L 4}=\left(\begin{array}{c}
u_{L 4} \\
d_{L 4}
\end{array}\right) ; \quad d_{L 5}, d_{R 5} ; \quad u_{L 5}, u_{R 5}
$$

The Yukawa couplings and the mass terms can be written in the basis:

$$
\begin{aligned}
& y_{i j}^{u} \tilde{\varphi} \overline{q_{L i}} u_{R j}+y_{i j}^{d} \varphi \overline{q_{L i}} d_{R j}+y_{4 j}^{u} \tilde{\varphi} \overline{q_{L 4}} u_{R j}+y_{4 j}^{d} \varphi \overline{q_{L 4}} d_{R j}+y_{i 5}^{u} \tilde{\varphi} \overline{q_{L i}} u_{R 5}+y_{i 5}^{d} \varphi \overline{q_{L i}} d_{R 5} \\
& +M_{q} \overline{q_{L 4}} q_{R 4}+M_{t^{\prime \prime}} \overline{u_{L 5}} u_{R 5}+M_{b^{\prime \prime}} \overline{d_{L 5}} d_{R 5}+\text { h.c. }
\end{aligned}
$$

with $i, j=1,2,3$. In this way, the non-zero couplings of the doublet with SM families $y_{42}^{d}, y_{41}^{u} \neq 0$ can cancel the discrepancy among the determinations of $\left|V_{u s}\right|$ obtained from leptonic and semileptonic kaon decays and the mixing with the first family of a down-type or up-type singlet (or both of them) $y_{15}^{d}, y_{15}^{u} \neq 0$ would remove the discrepancy between the determination obtained from beta decays with the determinations from kaon decays. The anomalies are explained with:

$$
V_{R 4 u}^{*} V_{R 4 s}=-0.97(36) \times 10^{-3}, \quad\left|V_{L 5 d}\right|^{2}+\left|V_{L 5 u}\right|^{2}=1.78(55) \times 10^{-3}
$$

with $V_{\text {Lus }}=0.22423(36)$. Here, analogously to eqs. (4.150), (6.10), we have:

$$
V_{L 5 d} \approx-\frac{y_{15}^{d *} v_{w}}{M_{b^{\prime \prime}}}, \quad V_{L 5 u} \approx-\frac{y_{15}^{u *} v_{w}}{M_{t^{\prime \prime}}}, \quad V_{R 4 u} \approx-\frac{y_{41}^{u} v_{w}}{M_{q}}, \quad V_{R 4 s} \approx-\frac{y_{42}^{d} v_{w}}{M_{q}}
$$

and similarly for the other elements. Then, also in this case the masses of the extra vectorlike family cannot exceed few $\mathrm{TeV}$ in order to mantain perturbativity. In fact, for example in order to have $\left|V_{R 4 u}\right| \approx\left|V_{R 4 s}\right| \gtrsim 0.025$ it should be that $M_{q} \lesssim 7 \mathrm{TeV}$ to have $\left|y_{15}^{d, u}\right|<1$.

Regarding weak charged currents, both $\mathrm{LH}$ and $\mathrm{RH}$ states interact with $W$-boson, with mixing matrices $V_{\mathrm{CKM}, \mathrm{L}}$ and $V_{\mathrm{CKM}, \mathrm{R}}$, which are not unitary. $V_{\mathrm{CKM}, \mathrm{R}}$ is generated by the mixing of the first three $\mathrm{RH}$ families with the vector-like weak doublets, as in the previous case. As regards $V_{\mathrm{CKM}, \mathrm{L}}$, analogously to eq. (5.8), the fifth row of the enlarged CKM matrix has mixing elements:

$$
V_{L t^{\prime \prime} d} \approx-V_{L 5 u}, \quad V_{L t^{\prime \prime} s} \approx-V_{L 5 u} V_{L u s}-V_{L 5 c} V_{L c s}-V_{L 5 t} V_{L t s}, \quad V_{L t^{\prime \prime} b} \approx-V_{L 5 t}
$$

generated by the mixing of the first three LH families with the vector-like up-type isosinglet. As for the elements of the fifth column, analogously to eq. (4.18), (4.19), (4.20), the elements 


\begin{tabular}{|ll|}
\hline Process & Constraint \\
\hline$K^{+} \rightarrow \pi^{+} \nu \bar{\nu}$ & $\left|V_{R 4 s}^{*} V_{R 4 d}+V_{L 5 s}^{*} V_{L 5 d}\right|<1.9 \times 10^{-5}$ \\
$K_{\mathrm{L}} \rightarrow \pi^{0} \nu \bar{\nu}$ & $\left|\operatorname{Im}\left(V_{R 4 s}^{*} V_{R 4 d}+V_{L 5 s}^{*} V_{L 5 d}\right)\right|<2.2 \times 10^{-5}$ \\
$K_{\mathrm{L}} \rightarrow \pi^{0} e^{+} e^{-}$ & $\left|\operatorname{Im}\left(V_{R 4 s}^{*} V_{R 4 d}+V_{L 5 s}^{*} V_{L 5 d}\right)\right|<1.7 \times 10^{-5}$ \\
$K_{\mathrm{L}} \rightarrow \mu^{+} \mu^{-}$ & $-0.3 \times 10^{-5}<\left|\operatorname{Re}\left(V_{L 5 s}^{*} V_{L 5 d}-V_{R 4 s}^{*} V_{R 4 d}\right)\right|<1.1 \times 10^{-5}$ \\
$K_{S} \rightarrow \mu^{+} \mu^{-}$ & $\left.\mid \operatorname{Im}\left(V_{L 5 s}^{*} V_{L 5 d}\right)-V_{R 4 s}^{*} V_{R 4 d}\right) \mid<4.8 \times 10^{-5}$ \\
\hline$B^{ \pm} \rightarrow \pi^{ \pm} \ell^{+} \ell^{-}$ & $\left|V_{L 5 b} V_{L 5 d}^{*}+V_{R 4 b} V_{R 4 d}^{*}\right|<2.5 \times 10^{-4}$ \\
$B^{0} \rightarrow \mu^{+} \mu^{-}$ & $\left|V_{L 5 b} V_{L 5 d}^{*}-V_{R 4 b} V_{R 4 d}^{*}\right|<2.2 \times 10^{-4}$ \\
\hline$Z \rightarrow b \bar{b}$ & $\left|V_{L 5 b}\right|^{2}+0.18\left|V_{R 4 b}\right|^{2}<1.0 \times 10^{-3}$ \\
\hline
\end{tabular}

Table 9. Limits on the mixing of the SM three families with the extra vector-like weak doublet $q_{L 4}, q R 4$ and the weak singlets $u_{L 5}, u_{R 5}, d_{L 5}, d_{R 5}$. See the text for constraints obtained from neutral mesons mixing and $Z$ decay into hadrons.

generated by the mixing of the first three LH families with the vector-like down-type isosinglet are:

$$
V_{L u b^{\prime \prime}} \approx-V_{L 5 d}^{*}, \quad V_{L c b^{\prime \prime}} \approx-V_{L 5 d}^{*} V_{L c d}-V_{L 5 s}^{*} V_{L c s}-V_{L 5 b}^{*} V_{L c b}, \quad V_{t b^{\prime \prime}} \approx-V_{L 5 b}^{*}
$$

while the mixings $V_{L \alpha b^{\prime}}, V_{L t^{\prime} \alpha}$ with the vector-like isodoublet are negligibly small.

As regards experimental constraints, the upper bounds from flavour changing decays of mesons are the same as in section 4, 5, 6, applied to vector and axial couplings of semileptonic and leptonic decays respectively, as shown in table 9. Regarding neutral mesons mixing, tree level and loop level contributions from both $\mathrm{LH}$ and $\mathrm{RH}$ currents are present, including mixed contributions. By taking $M_{b^{\prime \prime}}=M_{t^{\prime \prime}}=M_{q}=M$ for simplicity, the new additional terms in mixing mass of $\bar{K}^{0} \rightarrow K^{0}$ transition would be:

$$
\begin{gathered}
M_{12}^{\text {new }}=\frac{1}{3} f_{K}^{2} m_{K^{0}}\left\{\frac{G_{F}}{\sqrt{2}}\left[\left(V_{L 5 d}^{*} V_{L 5 s}\right)^{2}+\left(V_{R 4 d}^{*} V_{R 4 s}\right)^{2}-27.5\left(V_{R 4 d}^{*} V_{R 4 s}\right)\left(V_{L 5 d}^{*} V_{L 5 s}\right)\right]+\right. \\
\frac{G_{F}^{2}}{16 \pi^{2}} M^{2}\left[\left(V_{L 5 d}^{*} V_{L 5 s}\right)^{2}+2\left(V_{R 4 d}^{*} V_{R 4 s}\right)^{2}-54.9\left(V_{R 4 d}^{*} V_{R 4 s}\right)\left(V_{L 5 d}^{*} V_{L 5 s}\right)+\right. \\
\left.\left.+\left(\left|V_{L 5 u}\right|^{2} V_{L u s}+V_{L 5 u}^{*} V_{L 5 c}+V_{L 5 u}^{*} V_{L 5 t} V_{L t s}\right)^{2}\right]\right\}
\end{gathered}
$$

(where we neglected terms growing logarithmically with the mass of the extra quarks) and for $D^{0}$ mesons system:

$$
\begin{gathered}
M_{12 \text { new }}^{D}=\frac{1}{3} f_{D}^{2} m_{D^{0}}\left\{\frac{G_{F}}{\sqrt{2}}\left[\left(V_{L 5 u}^{*} V_{L 5 c}\right)^{2}+\left(V_{R 4 u}^{*} V_{R 4 c}\right)^{2}-3.65\left(V_{R 4 u}^{*} V_{R 4 c}\right)\left(V_{L 5 u}^{*} V_{L 5 c}\right)\right]+\right. \\
\frac{G_{F}^{2}}{16 \pi^{2}} M^{2}\left[\left(V_{L 5 u}^{*} V_{L 5 c}\right)^{2}+2\left(V_{R 4 u}^{*} V_{R 4 c}\right)^{2}-7.3\left(V_{R 4 u}^{*} V_{R 4 c}\right)\left(V_{L 5 u}^{*} V_{L 5 c}\right)+\right. \\
\left.\left.+\left(\left|V_{L 5 d}\right|^{2} V_{L c d}^{*}+V_{L 5 d}^{*} V_{L 5 s}+V_{L 5 d}^{*} V_{L 5 b} V_{L c b}^{*}\right)^{2}\right]\right\}
\end{gathered}
$$




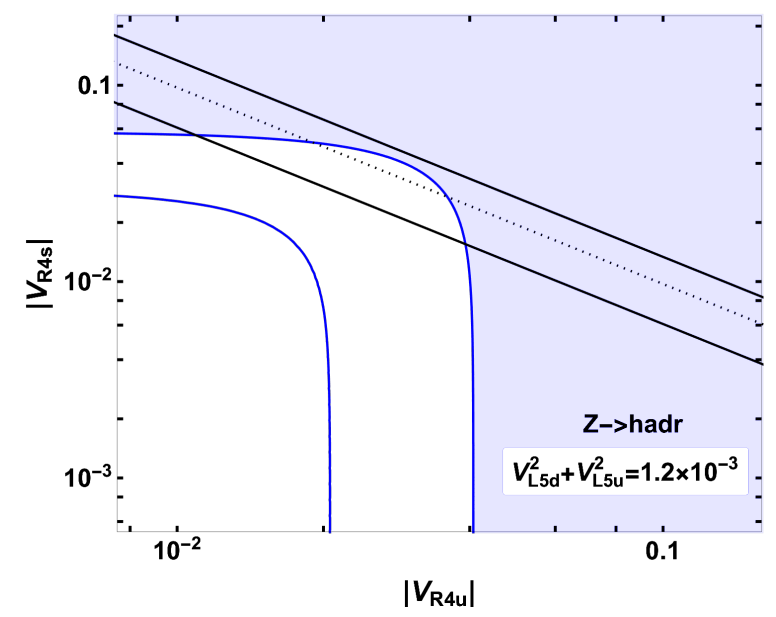

Figure 20. Excluded area in the $\left|V_{R 4 u}\right|$ and $\left|V_{R 4 s}\right|$ parameter space. The blue area is excluded by experimental constraints from $Z$-boson decay rate into hadrons (6.72) using $\left|V_{L 5 d}\right|^{2}+\left|V_{L 5 u}\right|^{2}=$ $1.2 \times 10^{-3}$. The blue curve indicates where the bound is shifted if the condition $\left|V_{L 5 d}\right|^{2}+\left|V_{L 5 u}\right|^{2}=$ $1.78 \times 10^{-3}(6.66)$ is used. The black curve stands for the solution $\left|V_{R 4 u}^{*} V_{R 4 s}\right|=0.97(36) \times 10^{-3}$ in eq. (6.66).

and similarly for neutral B-mesons systems. Therefore, in this case there can also be cancellations. However, also constraints on flavour conserving processes should be taken into account. The results on $Z$-boson decay rate into hadrons give the bound (see sections $4.4,5.3,6.2)$ :

$$
\begin{aligned}
& \left|V_{R 4 u}\right|^{2}+\left|V_{R 4 c}\right|^{2}+0.50\left(\left|V_{R 4 s}\right|^{2}+\left|V_{R 4 d}\right|^{2}+\left|V_{R 4 b}\right|^{2}\right)+ \\
& \quad+2.72\left(\left|V_{L 5 d}\right|^{2}+\left|V_{L 5 s}\right|^{2}+\left|V_{L 5 b}\right|^{2}\right)+2.24\left(\left|V_{L 5 u}\right|^{2}+\left|V_{L 5 c}\right|^{2}\right)<4.4 \times 10^{-3}
\end{aligned}
$$

Figure 20 shows the parameter space in a simplified case in which $y_{41}^{d}=y_{25}^{d}=y_{42}^{u}=$ $y_{25}^{u}=0$ in eq. (6.65), corresponding to $V_{R 4 d}=V_{L 5 s}=V_{R 4 c}=V_{L 5 c}=0$. The blue area is excluded by the condition (6.72) using the conservative choice $\left|V_{L 5 d}\right|^{2}+\left|V_{L 5 u}\right|^{2}=1.2 \times 10^{-3}$ for the solution in eq. (6.66). The solution $\left|V_{R 4 u}^{*} V_{R 4 s}\right|=0.97(36) \times 10^{-3}$ in eq. (6.66) is indicated by the black curve. In this scenario with $V_{R 4 d}=V_{L 5 s}=V_{R 4 c}=V_{L 5 c}=0$, the constraint from neutral $D$-mesons mixing would give (4.105):

$$
\left|V_{L u b^{\prime \prime}} V_{L c b^{\prime \prime}}^{*}\right| \approx\left|V_{L 5 d}\right|^{2}\left|V_{c d}\right|<3.9 \times 10^{-4}\left[\frac{1 \mathrm{TeV}}{M_{b^{\prime \prime}}}\right], \quad\left|V_{L 5 d}\right|<0.042\left[\frac{1 \mathrm{TeV}}{M_{b^{\prime \prime}}}\right]^{1 / 2}
$$

Kaon mixing would receive a real contribution at loop level giving the constraint (5.23):

$$
\left.\left|V_{L t^{\prime \prime} d} V_{L t^{\prime \prime} s}^{*}\right| \approx|| V_{L 5 u}\right|^{2} V_{L u s}+V_{L 5 u} V_{L 5 t}^{*} V_{L t s} \mid<5.2 \times 10^{-4}\left[\frac{1 \mathrm{TeV}}{M_{b^{\prime \prime}}}\right]
$$

which together with the constraints from B-mesons decays and mixing (5.31) gives:

$$
\left|V_{L 5 u}\right| \lesssim 0.049\left[\frac{1 \mathrm{TeV}}{M_{t^{\prime \prime}}}\right]^{1 / 2}
$$

This means that, in order to have $\left|V_{L 5 d}\right|^{2}+\left|V_{L 5 u}\right|^{2}>1.2 \times 10^{-3}$ (6.66), it should be for example $M_{b^{\prime \prime}} \approx M_{t^{\prime \prime}}<3.5 \mathrm{TeV}$. 


\section{Conclusion}

Present situation of the determination of the CKM matrix elements is very intriguing. From one side, there is a controversy in the determination of $\left|V_{u s}\right|$. Namely, given the present experimental accuracy in kaon leptonic and semileptonic decays and present theoretical precision in the calculation of form factors (lattice QCD), there is about $3 \sigma$ tension between the $\left|V_{u s}\right|$ values extracted from $K \ell 3$ (determination A) and $K \mu 2$ (determination B) decays, as discussed in section 2. Although the recent high precision calculations in the kaon decays $K \ell 3[38]$ and $K \mu 2$ [35] demonstrate that the radiative corrections should not be responsible for it, this tension per sè cannot be considered that alarming since still there can be some theoretical loopholes in the interpretation of the lattice QCD results, or perhaps some unfixed systematics in the measurements of the kaon decay rates. In this case, the real value of $\left|V_{u s}\right|$ could be near the average $A+B$ between the two above determinations.

On the other side, the recent calculations of the short distance radiative corrections in the neutron $\beta$-decay with improved hadronic uncertainties [26, 27] leads to higher accuracy in the determination of $\left|V_{u d}\right|$ from superallowed $0^{+}-0^{+}$nuclear transitions (determination C). The obtained $\left|V_{u d}\right|$ value, in combination with the determinations of $\left|V_{u s}\right|$, indicates towards a violation of the CKM unitarity at about $3 \sigma$ level. Namely, there appears a significant deficit $\delta_{\mathrm{CKM}}$ in the first row unitarity, as given by eq. (2.1).

These anomalies, if confirmed with future high precision data, would indicate towards some new physics beyond the Standard Model. In ref. [1] we pointed out that the anomalies could be originated by mixing of ordinary light quarks to some extra vector-like quarks with masses at the $\mathrm{TeV}$ scale. In this paper we gave more detailed study of these scenarios, analyzing one by one the implications of vector-like quarks in the weak isosinglet or isodoublet representations. By introducing a weak isosinglet quark of the up ( $U$-type) or down ( $D$-type) quarks, one can explain the CKM unitarity anomaly, i.e. deficit of the first row unitarity (2.1), but the tension between the two determinations of $\left|V_{u s}\right|$ cannot be explained in this case. However, if the latter discrepancy is taken seriously one has to look for a solution addressing the whole situation. By introducing a weak isodoublet $(Q$-type) of quarks one could potentially explain both anomalies.

However, there are strong phenomenological limits on the hypothetical vector-like quarks: participation of vector-like quark species in the quark mixing induces flavor changing effects at the tree level as well as via box diagrams involving heavy species. In fact, we show that the latter give a bigger contribution than the tree level one if the masses of heavy quarks are larger than $3 \mathrm{TeV}$ or so. However, tree level effects are independently testable in $Z$-boson physics since $Z$-boson acquires small flavor non-diagonal couplings with quarks while also its flavor-diagonal couplings get weak isospin violating contributions which can be confronted with the limits on many observables related to $Z$-boson physics.

Two approaches to the above anomalies can be considered. The incompatibility between two determinations A and B from kaon physics may be attributed to some uncertainties which perhaps will disappear with more precise calculations. Neglecting this problem, one can focus instead on the unitarity violation problem, i.e. on the unitarity deficit which emerges by confronting the average $A+B$ value of $\left|V_{u s}\right|$ obtained from kaons, with the value of $\left|V_{u d}\right|$ extracted from superallowed nuclear transitions. 
The just mentioned scenarios involving extra isosinglets quarks are on this line. Namely, the CKM problem can be solved provided that the first family of quarks has a mixing $\left|V_{u b^{\prime}}\right| \simeq 0.04$ with extra $D$-type quark $b^{\prime}$, or alternatively the same size mixing $V_{t^{\prime} d}$ with $U$-type quark $t^{\prime}$. For this scenario the most severe limits come from the flavor changing phenomena, but comparable limits emerge from the precision data on $Z$-boson decays. There still remains some available parameter space, although not very large. In fact, we show that constraints from $K^{0}-\bar{K}^{0}$ and $D^{0}-\bar{D}^{0}$ systems become more stringent for larger masses of $b^{\prime}$ or $t^{\prime}$. In particular, e.g. for $M_{b^{\prime}}>1.5 \mathrm{TeV}$ they would exclude the possibility of having extra mixing as large as $\left|V_{u b^{\prime}}\right| \sim 0.04$ if the unitarity deficit is due to a down-type species or, in the case of up-type extra quark, $M_{t^{\prime}}>2.5 \mathrm{TeV}$ would exclude the possibility of having the extra mixing $\left|V_{t^{\prime} d}\right| \sim 0.04$ (see sections 4.5, 5.4). Therefore, we claim that if the CKM unitarity anomaly is due to the mixing with extra isosinglet ( $U$ or $D$ type) quarks, then "4th family" states $b^{\prime}$ or $t^{\prime}$ should be discovered with a mass below a couple of $\mathrm{TeV}$ or so.

As we noted above, both $V_{u s}$ and CKM unitarity anomalies can be solved by introducing $Q$-type extra quarks having substantial mixings with both 1st and 2nd families of the normal quarks. In fact, kaon semileptonic decays $K \ell 3$ measure the quark vector current coupling to $W$-boson while leptonic decays $K \mu 2$ measure the axial current coupling. While both vector and axial couplings should be identical in the SM frames where $W$-boson couples solely to LH quarks, their difference can be induced via the mixing with weak isodoublet $Q$-type quarks. The latter in fact induces some small $W$-boson couplings with the RH quarks which can be at the origin of the discrepancy between the two determinations A and $\mathrm{B}$ of $\left|V_{u s}\right|$ element. However, we show that this solution with only one $Q$-type species is fully excluded by the flavor changing limits together with $Z$ boson physics and electroweak low energy observables (section 6.4).

This brings us to conclude that the full solution cannot be achieved by introducing the only one species of extra $Q$-type quarks: it should be complemented by isosinglet quarks of $U$ or/and $D$ type, or by another isodoublet species. In this case somewhat larger available parameter space can be found with the present limits on flavor-changing phenomena and on anomalous $Z$-couplings. However, these scenarios are testable with the future experimental limits (section 6.5). In particular, in the scenario with two isodoublets, flavour changing limits can be softened, and then the masses of the extra quarks would not be strictly constrained, apart from a perturbativity bound (Yukawa couplings less than 1), which implies $M_{q}<7 \mathrm{TeV}$ or so. However, in this case the main issue would come from limits in $Z$-boson physics. In the case of one extra isodoublet with isosinglets (up or/and down type), also constraints from flavour changing phenomena should be considered. We conclude that if the CKM anomalies are due to extra vector-like quarks, then "4th family" quarks should be discovered with masses of few $\mathrm{TeV}$, and anomalous $Z$-couplings should be detected by improving the experimental precision.

For the minimality reasons, we did not consider extra vector-like species of leptons. However, if they also exist at the $\mathrm{TeV}$ scale and have the same size mixings with the electron and muon, then the experimental limits from the flavor changing phenomena involving leptons or limits on $Z$-boson flavor-nondiagonal couplings as $Z \bar{e} \mu$ would be much more stringent. 


\section{Acknowledgments}

The work of B.B. was supported by the ERC research grant NEO-NAT no. 669668. The work of Z.B. was supported in part by Ministero dell'Istruzione, Università e della Ricerca (MIUR) under the program PRIN 2017, grant no. 2017X7X85K "The Dark Universe: A Synergic Multimessenger Approach", and in part by Shota Rustaveli National Science Foundation (SRNSF) of Georgia, grant DI-18-335 "New Theoretical Models for Dark Matter Exploration".

Open Access. This article is distributed under the terms of the Creative Commons Attribution License (CC-BY 4.0), which permits any use, distribution and reproduction in any medium, provided the original author(s) and source are credited.

\section{References}

[1] B. Belfatto, R. Beradze and Z. Berezhiani, The CKM unitarity problem: A trace of new physics at the TeV scale?, Eur. Phys. J. C 80 (2020) 149 [arXiv: 1906.02714] [InSPIRE].

[2] B. Belfatto and Z. Berezhiani, How light the lepton flavor changing gauge bosons can be, Eur. Phys. J. C 79 (2019) 202 [arXiv:1812.05414] [InSPIRE].

[3] Y. Grossman, E. Passemar and S. Schacht, On the Statistical Treatment of the Cabibbo Angle Anomaly, JHEP 07 (2020) 068 [arXiv:1911.07821] [INSPIRE].

[4] F. Giacosa and G. Pagliara, Measurement of the neutron lifetime and inverse quantum Zeno effect, Phys. Rev. D 101 (2020) 056003 [arXiv:1906.10024] [INSPIRE].

[5] A.M. Coutinho, A. Crivellin and C.A. Manzari, Global Fit to Modified Neutrino Couplings and the Cabibbo-Angle Anomaly, Phys. Rev. Lett. 125 (2020) 071802 [arXiv:1912.08823] [INSPIRE].

[6] K. Cheung, W.-Y. Keung, C.-T. Lu and P.-Y. Tseng, Vector-like Quark Interpretation for the CKM Unitarity Violation, Excess in Higgs Signal Strength, and Bottom Quark Forward-Backward Asymmetry, JHEP 05 (2020) 117 [arXiv:2001.02853] [INSPIRE].

[7] A. Crivellin and M. Hoferichter, $\beta$ Decays as Sensitive Probes of Lepton Flavor Universality, Phys. Rev. Lett. 125 (2020) 111801 [arXiv:2002.07184] [INSPIRE].

[8] M. Endo and S. Mishima, Muon $g-2$ and CKM unitarity in extra lepton models, JHEP 08 (2020) 004 [arXiv: 2005. 03933] [inSPIRE].

[9] B. Capdevila, A. Crivellin, C.A. Manzari and M. Montull, Explaining $b \rightarrow s \ell^{+} \ell^{-}$and the Cabibbo angle anomaly with a vector triplet, Phys. Rev. D 103 (2021) 015032 [arXiv: 2005.13542] [INSPIRE].

[10] A. Crivellin, F. Kirk, C.A. Manzari and M. Montull, Global Electroweak Fit and Vector-Like Leptons in Light of the Cabibbo Angle Anomaly, JHEP 12 (2020) 166 [arXiv:2008.01113] [INSPIRE].

[11] M. Kirk, Cabibbo anomaly versus electroweak precision tests: An exploration of extensions of the Standard Model, Phys. Rev. D 103 (2021) 035004 [arXiv:2008.03261] [INSPIRE].

[12] C.A. Manzari, A.M. Coutinho and A. Crivellin, Modified lepton couplings and the Cabibbo-angle anomaly, PoS LHCP2020 (2021) 242 [arXiv: 2009. 03877] [INSPIRE]. 
[13] A.K. Alok, A. Dighe, S. Gangal and J. Kumar, The role of non-universal Z couplings in explaining the Vus anomaly, Nucl. Phys. B 971 (2021) 115538 [arXiv:2010.12009] [INSPIRE].

[14] A. Crivellin, C.A. Manzari, M. Alguero and J. Matias, Combined Explanation of the $Z \rightarrow b \bar{b}$ Forward-Backward Asymmetry, the Cabibbo Angle Anomaly, $\tau \rightarrow \mu \nu \nu$ and $b \rightarrow s \ell^{+} \ell^{-}$Data, Phys. Rev. Lett. 127 (2021) 011801 [arXiv: 2010.14504] [INSPIRE].

[15] A. Crivellin, F. Kirk, C.A. Manzari and L. Panizzi, Searching for lepton flavor universality violation and collider signals from a singly charged scalar singlet, Phys. Rev. D 103 (2021) 073002 [arXiv: 2012.09845] [INSPIRE].

[16] A. Crivellin, M. Hoferichter and C.A. Manzari, Fermi Constant from Muon Decay Versus Electroweak Fits and Cabibbo-Kobayashi-Maskawa Unitarity, Phys. Rev. Lett. 127 (2021) 071801 [arXiv: 2102.02825] [INSPIRE].

[17] M. Moulson, Experimental determination of $V_{u s}$ from kaon decays, PoS CKM2016 (2017) 033 [arXiv: 1704.04104] [INSPIRE].

[18] Flavour lattice Averaging Group collaboration, FLAG Review 2019: Flavour Lattice Averaging Group (FLAG), Eur. Phys. J. C 80 (2020) 113 [arXiv:1902.08191] [INSPIRE].

[19] Fermilab Lattice and MILC collaborations, $\left|V_{u s}\right|$ from $K_{\ell 3}$ decay and four-flavor lattice QCD, Phys. Rev. D 99 (2019) 114509 [arXiv:1809.02827] [InSPIRE].

[20] Particle Data Group collaboration, Review of Particle Physics, PTEP 2020 (2020) 083C01 [INSPIRE].

[21] J.C. Hardy and I.S. Towner, Superallowed $0^{+} \rightarrow 0^{+}$nuclear $\beta$ decays: 2014 critical survey, with precise results for $V_{u d}$ and CKM unitarity, Phys. Rev. C 91 (2015) 025501 [arXiv: 1411.5987] [INSPIRE].

[22] J.C. Hardy and I.S. Towner, Superallowed $0^{+} \rightarrow 0^{+}$nuclear $\beta$ decays: 2020 critical survey, with implications for $V_{u d}$ and CKM unitarity, Phys. Rev. C 102 (2020) 045501 [INSPIRE].

[23] J.C. Hardy and I.S. Towner, $\left|V_{u d}\right|$ from nuclear $\beta$ decays, PoS CKM2016 (2016) 028 [INSPIRE].

[24] MuLan collaboration, Detailed Report of the MuLan Measurement of the Positive Muon Lifetime and Determination of the Fermi Constant, Phys. Rev. D 87 (2013) 052003 [arXiv:1211.0960] [INSPIRE].

[25] W.J. Marciano and A. Sirlin, Improved calculation of electroweak radiative corrections and the value of $V_{u d}$, Phys. Rev. Lett. 96 (2006) 032002 [hep-ph/0510099] [INSPIRE].

[26] C.-Y. Seng, M. Gorchtein, H.H. Patel and M.J. Ramsey-Musolf, Reduced Hadronic Uncertainty in the Determination of $V_{u d}$, Phys. Rev. Lett. 121 (2018) 241804 [arXiv: 1807.10197] [INSPIRE].

[27] A. Czarnecki, W.J. Marciano and A. Sirlin, Radiative Corrections to Neutron and Nuclear Beta Decays Revisited, Phys. Rev. D 100 (2019) 073008 [arXiv:1907.06737] [InSPIRE].

[28] C.-Y. Seng, X. Feng, M. Gorchtein and L.-C. Jin, Joint lattice QCD-dispersion theory analysis confirms the quark-mixing top-row unitarity deficit, Phys. Rev. D 101 (2020) 111301 [arXiv: 2003.11264] [INSPIRE].

[29] L. Hayen, Standard model $\mathcal{O}(\alpha)$ renormalization of $g_{A}$ and its impact on new physics searches, Phys. Rev. D 103 (2021) 113001 [arXiv:2010.07262] [INSPIRE]. 
[30] K. Shiells, P.G. Blunden and W. Melnitchouk, Electroweak axial structure functions and improved extraction of the Vud CKM matrix element, Phys. Rev. D 104 (2021) 033003 [arXiv: 2012.01580] [INSPIRE].

[31] I.S. Towner and J.C. Hardy, The evaluation of $V(u d)$ and its impact on the unitarity of the Cabibbo-Kobayashi-Maskawa quark-mixing matrix, Rept. Prog. Phys. 73 (2010) 046301 [INSPIRE].

[32] Z. Berezhiani, Neutron lifetime puzzle and neutron-mirror neutron oscillation, Eur. Phys. J. C 79 (2019) 484 [arXiv:1807.07906] [INSPIRE].

[33] A. Czarnecki, W.J. Marciano and A. Sirlin, Neutron Lifetime and Axial Coupling Connection, Phys. Rev. Lett. 120 (2018) 202002 [arXiv: 1802.01804] [INSPIRE].

[34] D. Pocanic et al., Precise measurement of the $\pi^{+} \rightarrow \pi^{0} e^{+} \nu$ branching ratio, Phys. Rev. Lett. 93 (2004) 181803 [hep-ex/0312030] [INSPIRE].

[35] M. Di Carlo et al., Light-meson leptonic decay rates in lattice QCD+QED, Phys. Rev. D 100 (2019) 034514 [arXiv: 1904.08731] [INSPIRE].

[36] Particle Data Group collaboration, Review of Particle Physics, Phys. Rev. D 98 (2018) 030001 [INSPIRE].

[37] S. Aoki et al., Review of lattice results concerning low-energy particle physics, Eur. Phys. J. C 77 (2017) 112 [arXiv:1607.00299] [INSPIRE].

[38] C.-Y. Seng, D. Galviz, M. Gorchtein and U.G. Meißner, High-precision determination of the Ke3 radiative corrections, Phys. Lett. B 820 (2021) 136522 [arXiv:2103.00975] [InSPIRE].

[39] G. Bregar and N.S.M. Borstnik, New experimental data for the quarks mixing matrix are in better agreement with the spin-charge-family theory predictions, Bled Workshops Phys. 15 (2014) 20 [arXiv: 1412.5866] [INSPIRE].

[40] S.L. Glashow, J. Iliopoulos and L. Maiani, Weak Interactions with Lepton-Hadron Symmetry, Phys. Rev. D 2 (1970) 1285 [InSPIRE].

[41] S.L. Glashow and S. Weinberg, Natural Conservation Laws for Neutral Currents, Phys. Rev. D 15 (1977) 1958 [InSPIRE].

[42] E.A. Paschos, Diagonal Neutral Currents, Phys. Rev. D 15 (1977) 1966 [InSPIRE].

[43] F. Gursey, P. Ramond and P. Sikivie, A Universal Gauge Theory Model Based on E6, Phys. Lett. B 60 (1976) 177 [inSPIRE].

[44] Y. Achiman and B. Stech, Quark Lepton Symmetry and Mass Scales in an E6 Unified Gauge Model, Phys. Lett. B 77 (1978) 389 [INSPIRE].

[45] Z.G. Berezhiani and G.R. Dvali, Possible solution of the hierarchy problem in supersymmetric grand unification theories, Bull. Lebedev Phys. Inst. 5 (1989) 55 [Kratk. Soobshch. Fiz. 5 (1989) 42] [INSPIRE].

[46] R. Barbieri, G.R. Dvali, A. Strumia, Z. Berezhiani and L.J. Hall, Flavor in supersymmetric grand unification: A Democratic approach, Nucl. Phys. B 432 (1994) 49 [hep-ph/9405428] [INSPIRE].

[47] Z. Berezhiani, SUSY SU(6) GIFT for doublet-triplet splitting and fermion masses, Phys. Lett. B 355 (1995) 481 [hep-ph/9503366] [INSPIRE].

[48] Z.G. Berezhiani, The Weak Mixing Angles in Gauge Models with Horizontal Symmetry: A New Approach to Quark and Lepton Masses, Phys. Lett. B 129 (1983) 99 [INSPIRE]. 
[49] S. Dimopoulos, Natural Generation of Fermion Masses, Phys. Lett. B 129 (1983) 417 [INSPIRE].

[50] Z.G. Berezhiani, Horizontal Symmetry and Quark-Lepton Mass Spectrum: The $\mathrm{SU}(5) \otimes \mathrm{SU}(3)_{H}$ Model, Phys. Lett. B 150 (1985) 177 [INSPIRE].

[51] J. Bagger, S. Dimopoulos, H. Georgi and S. Raby, Theories of Fermion Masses, SLAC-PUB-3342 (1984).

[52] Z.G. Berezhiani and M.Y. Khlopov, The Theory of broken gauge symmetry of families (in Russian), Sov. J. Nucl. Phys. 51 (1990) 739 [InSPIRE].

[53] Z.G. Berezhiani and M.Y. Khlopov, Physical and astrophysical consequences of breaking of the symmetry of families (in Russian), Sov. J. Nucl. Phys. 51 (1990) 935 [inSPIRE].

[54] S.F. King and G.G. Ross, Fermion masses and mixing angles from SU(3) family symmetry, Phys. Lett. B 520 (2001) 243 [hep-ph/0108112] [INSPIRE].

[55] Z. Berezhiani and A. Rossi, Predictive grand unified textures for quark and neutrino masses and mixings, Nucl. Phys. B 594 (2001) 113 [hep-ph/0003084] [INSPIRE].

[56] J.E. Kim, Weak Interaction Singlet and Strong CP Invariance, Phys. Rev. Lett. 43 (1979) 103 [INSPIRE].

[57] M.A. Shifman, A.I. Vainshtein and V.I. Zakharov, Can Confinement Ensure Natural CP Invariance of Strong Interactions?, Nucl. Phys. B 166 (1980) 493 [INSPIRE].

[58] Z.G. Berezhiani and M.Y. Khlopov, Physics of cosmological dark matter in the theory of broken family symmetry (in Russian), Sov. J. Nucl. Phys. 52 (1990) 60 [inSPIRE].

[59] Z.G. Berezhiani and M.Y. Khlopov, Cosmology of Spontaneously Broken Gauge Family Symmetry, Z. Phys. C 49 (1991) 73 [InSPIRE].

[60] Z.G. Berezhiani, A.S. Sakharov and M.Y. Khlopov, Primordial background of cosmological axions, Sov. J. Nucl. Phys. 55 (1992) 1063 [inSPIRE].

[61] A.E. Nelson, Naturally Weak CP-violation, Phys. Lett. B 136 (1984) 387 [InSPIRE].

[62] S.M. Barr, Solving the Strong CP Problem Without the Peccei-Quinn Symmetry, Phys. Rev. Lett. 53 (1984) 329 [INSPIRE].

[63] K.S. Babu and R.N. Mohapatra, A Solution to the Strong CP Problem Without an Axion, Phys. Rev. D 41 (1990) 1286 [inSPIRE].

[64] Z.G. Berezhiani, On the possibility of a solution to the strong CP problem without axion in a $\mathrm{SU}(3)_{H}$ family symmetry model, Mod. Phys. Lett. A 6 (1991) 2437 [INSPIRE].

[65] Z.G. Berezhiani, R.N. Mohapatra and G. Senjanović, Planck scale physics and solutions to the strong CP problem without axion, Phys. Rev. D 47 (1993) 5565 [hep-ph/9212318] [INSPIRE].

[66] Z.G. Berezhiani and J.L. Chkareuli, Low-energy horizontal symmetry of $\mathrm{SU}(3)_{H} \times \mathrm{U}(1)_{H}$ and $B-\bar{B}$ oscillation (in Russian), Sov. J. Nucl. Phys. 52 (1990) 383 [inSPIRE].

[67] S. Rajpoot, Seesaw Masses for Quarks and Leptons, Phys. Rev. D 36 (1987) 1479 [inSPIRE].

[68] A. Davidson and K.C. Wali, Universal Seesaw Mechanism?, Phys. Rev. Lett. 59 (1987) 393 [INSPIRE].

[69] Z.G. Berezhiani and R. Rattazzi, Universal seesaw and radiative quark mass hierarchy, Phys. Lett. B 279 (1992) 124 [INSPIRE]. 
[70] Z.G. Berezhiani and R. Rattazzi, Inverse hierarchy approach to fermion masses, Nucl. Phys. B 407 (1993) 249 [hep-ph/9212245] [InSPIRE].

[71] Z.G. Berezhiani, Predictive SUSY SO(10) model with very low tan Beta, Phys. Lett. B 355 (1995) 178 [hep-ph/9505384] [INSPIRE].

[72] G. Anderson, S. Raby, S. Dimopoulos, L.J. Hall and G.D. Starkman, A Systematic SO(10) operator analysis for fermion masses, Phys. Rev. D 49 (1994) 3660 [hep-ph/9308333] [INSPIRE].

[73] Z. Berezhiani and Z. Tavartkiladze, More missing VEV mechanism in supersymmetric SO(10) model, Phys. Lett. B 409 (1997) 220 [hep-ph/9612232] [INSPIRE].

[74] Y. Koide and H. Fusaoka, Top quark mass enhancement in a seesaw type quark mass matrix, Z. Phys. C 71 (1996) 459 [hep-ph/9505201] [INSPIRE].

[75] Y. Koide, Universal seesaw mass matrix model with an $S_{3}$ symmetry, Phys. Rev. D 60 (1999) 077301 [hep-ph/9905416] [INSPIRE].

[76] Z. Berezhiani and F. Nesti, Supersymmetric $\mathrm{SO}(10)$ for fermion masses and mixings: Rank-1 structures of flavor, JHEP 03 (2006) 041 [hep-ph/0510011] [INSPIRE].

[77] Z. Berezhiani, Unified picture of the particle and sparticle masses in SUSY GUT, Phys. Lett. B 417 (1998) 287 [hep-ph/9609342] [INSPIRE].

[78] A. Anselm and Z. Berezhiani, Weak mixing angles as dynamical degrees of freedom, Nucl. Phys. B 484 (1997) 97 [hep-ph/9605400] [INSPIRE].

[79] Z. Berezhiani and A. Rossi, Flavor structure, flavor symmetry and supersymmetry, Nucl. Phys. B Proc. Suppl. 101 (2001) 410 [hep-ph/0107054] [INSPIRE].

[80] L. Lavoura and J.P. Silva, Bounds on the mixing of the down type quarks with vector-like singlet quarks, Phys. Rev. D 47 (1993) 1117 [INSPIRE].

[81] K. Ishiwata, Z. Ligeti and M.B. Wise, New Vector-Like Fermions and Flavor Physics, JHEP 10 (2015) 027 [arXiv: 1506.03484] [INSPIRE].

[82] CMS collaboration, Search for vectorlike light-flavor quark partners in proton-proton collisions at $\sqrt{s}=8 \mathrm{TeV}$, Phys. Rev. D 97 (2018) 072008 [arXiv:1708.02510] [INSPIRE].

[83] G. Buchalla, A.J. Buras and M.E. Lautenbacher, Weak decays beyond leading logarithms, Rev. Mod. Phys. 68 (1996) 1125 [hep-ph/9512380] [INSPIRE].

[84] A.J. Buras, D. Buttazzo, J. Girrbach-Noe and R. Knegjens, $K^{+} \rightarrow \pi^{+} \nu \bar{\nu}$ and $K_{L} \rightarrow \pi^{0} \nu \bar{\nu}$ in the Standard Model: status and perspectives, JHEP 11 (2015) 033 [arXiv:1503.02693] [INSPIRE].

[85] J. Brod and M. Gorbahn, Electroweak Corrections to the Charm Quark Contribution to $K^{+} \rightarrow \pi^{+} \nu \bar{\nu}$, Phys. Rev. D 78 (2008) 034006 [arXiv:0805.4119] [inSPIRE].

[86] F. Mescia and C. Smith, Improved estimates of rare $K$ decay matrix-elements from Kl3 decays, Phys. Rev. D 76 (2007) 034017 [arXiv:0705.2025] [INSPIRE].

[87] NA62 collaboration, An investigation of the very rare $K^{+} \rightarrow \pi^{+} \nu \bar{\nu}$ decay, JHEP 11 (2020) 042 [arXiv: 2007.08218] [inSPIRE].

[88] A.J. Buras, Weak Hamiltonian, CP-violation and rare decays, in Les Houches Summer School in Theoretical Physics, Session 68: Probing the Standard Model of Particle Interactions, pp. 281-539 (1998) [hep-ph/9806471] [INSPIRE]. 
[89] A.J. Buras and R. Fleischer, Quark mixing, CP-violation and rare decays after the top quark discovery, Adv. Ser. Direct. High Energy Phys. 15 (1998) 65 [hep-ph/9704376] [InSPIRE].

[90] G. Isidori and R. Unterdorfer, On the short distance constraints from $K_{L, S} \rightarrow \mu^{+} \mu^{-}$, JHEP 01 (2004) 009 [hep-ph/0311084] [INSPIRE].

[91] V. Cirigliano, G. Ecker, H. Neufeld, A. Pich and J. Portoles, Kaon Decays in the Standard Model, Rev. Mod. Phys. 84 (2012) 399 [arXiv:1107.6001] [InSPIRE].

[92] LHCb collaboration, Constraints on the $K_{S}^{0} \rightarrow \mu^{+} \mu^{-}$Branching Fraction, Phys. Rev. Lett. 125 (2020) 231801 [arXiv: 2001.10354] [INSPIRE].

[93] T. Inami and C.S. Lim, Effects of Superheavy Quarks and Leptons in Low-Energy Weak Processes $K_{L} \rightarrow \mu \bar{\mu}, K^{+} \rightarrow \pi^{+} \nu \bar{\nu}$ and $K^{0} \leftrightarrow \bar{K}^{0}$, Prog. Theor. Phys. 65 (1981) 297 [Erratum ibid. 65 (1981) 1772] [INSPIRE].

[94] Z. Bai, N.H. Christ, T. Izubuchi, C.T. Sachrajda, A. Soni and J. Yu, $K_{L}-K_{S}$ Mass Difference from Lattice QCD, Phys. Rev. Lett. 113 (2014) 112003 [arXiv:1406.0916] [INSPIRE].

[95] Z. Bai, N.H. Christ and C.T. Sachrajda, The $K_{L}-K_{S}$ Mass Difference, EPJ Web Conf. 175 (2018) 13017 [INSPIRE].

[96] G.C. Branco, L. Lavoura and J.P. Silva, CP Violation, Int. Ser. Monogr. Phys. 103 (1999) 1 [INSPIRE].

[97] A.A. Petrov, Dipenguin-like contributions to $D^{0}-\bar{D}^{0}$ mixing, AIP Conf. Proc. 432 (1998) 852 [hep-ph/9712279] [INSPIRE].

[98] H.-Y. Cheng and C.-W. Chiang, Long-Distance Contributions to $D^{0}-\bar{D}^{0}$ Mixing Parameters, Phys. Rev. D 81 (2010) 114020 [arXiv:1005.1106] [InSPIRE].

[99] LHCb collaboration, Observation of the Mass Difference Between Neutral Charm-Meson Eigenstates, Phys. Rev. Lett. 127 (2021) 111801 [arXiv:2106.03744] [INSPIRE].

[100] C. Bobeth, M. Gorbahn, T. Hermann, M. Misiak, E. Stamou and M. Steinhauser, $B_{s, d} \rightarrow l^{+} l^{-}$in the Standard Model with Reduced Theoretical Uncertainty, Phys. Rev. Lett. 112 (2014) 101801 [arXiv:1311.0903] [INSPIRE].

[101] ATLAS collaboration, Study of the rare decays of $B_{s}^{0}$ and $B^{0}$ mesons into muon pairs using data collected during 2015 and 2016 with the ATLAS detector, JHEP 04 (2019) 098 [arXiv: 1812.03017] [INSPIRE].

[102] BeLLE collaboration, Improved measurement of the electroweak penguin process $B \rightarrow X_{s} l^{+} l^{-}$, Phys. Rev. D 72 (2005) 092005 [hep-ex/0503044] [INSPIRE].

[103] BABAR collaboration, Measurement of the $B \rightarrow X_{s} l^{+} l^{-}$branching fraction and search for direct CP-violation from a sum of exclusive final states, Phys. Rev. Lett. 112 (2014) 211802 [arXiv: 1312.5364] [INSPIRE].

[104] T. Huber, T. Hurth and E. Lunghi, Inclusive $\bar{B} \rightarrow X_{s} \ell^{+} \ell^{-}$: complete angular analysis and a thorough study of collinear photons, JHEP 06 (2015) 176 [arXiv: 1503.04849] [INSPIRE].

[105] A. Ghinculov, T. Hurth, G. Isidori and Y.P. Yao, The Rare decay $B \rightarrow X_{s} l^{+} l^{-}$to $N N L L$ precision for arbitrary dilepton invariant mass, Nucl. Phys. B 685 (2004) 351 [hep-ph/0312128] [INSPIRE].

[106] LHCb collaboration, First measurement of the differential branching fraction and $C P$ asymmetry of the $B^{ \pm} \rightarrow \pi^{ \pm} \mu^{+} \mu^{-}$decay, JHEP 10 (2015) 034 [arXiv:1509.00414] [INSPIRE]. 
[107] A. Ali, A.Y. Parkhomenko and A.V. Rusov, Precise Calculation of the Dilepton Invariant-Mass Spectrum and the Decay Rate in $B^{ \pm} \rightarrow \pi^{ \pm} \mu^{+} \mu^{-}$in the SM, Phys. Rev. D 89 (2014) 094021 [arXiv:1312.2523] [INSPIRE].

[108] Fermilab Lattice and MILC collaborations, $B \rightarrow \pi \ell \ell$ form factors for new-physics searches from lattice QCD, Phys. Rev. Lett. 115 (2015) 152002 [arXiv:1507.01618] [INSPIRE].

[109] Belle collaboration, Search for $B \rightarrow \pi \ell^{+} \ell^{-}$Decays at Belle, Phys. Rev. D 78 (2008) 011101 [arXiv: 0804. 3656] [INSPIRE].

[110] BABAR collaboration, Search for the rare decays $B \rightarrow \pi \ell^{+} \ell^{-}$and $B^{0} \rightarrow \eta \ell^{+} \ell^{-}$, Phys. Rev. $D 88$ (2013) 032012 [arXiv: 1303.6010] [INSPIRE].

[111] ALEPH, DELPHI, L3, OPAL and SLD collaborations, LEP Electroweak Working Group, SLD Electroweak Group and SLD Heavy Flavour Group, Precision electroweak measurements on the $Z$ resonance, Phys. Rept. 427 (2006) 257 [hep-ex/0509008] [InSPIRE]. 